\title{
Quantum Multicriticality near the Dirac-Semimetal to Band-Insulator Critical Point in Two Dimensions: A Controlled Ascent from One Dimension
}

\author{
Bitan Roy ${ }^{1}$ and Matthew S. Foster ${ }^{1,2}$ \\ ${ }^{1}$ Department of Physics and Astronomy, Rice University, Houston, Texas 77005, USA \\ ${ }^{2}$ Rice Center for Quantum Materials, Rice University, Houston, Texas 77005, USA
}

(Received 12 June 2017; revised manuscript received 14 December 2017; published 26 March 2018)

We compute the effects of generic short-range interactions on gapless electrons residing at the quantum critical point separating a two-dimensional Dirac semimetal and a symmetry-preserving band insulator. The electronic dispersion at this critical point is anisotropic $\left(E_{\mathbf{k}}= \pm \sqrt{v^{2} k_{x}^{2}+b^{2} k_{y}^{2 n}}\right.$ with $\left.n=2\right)$, which results in unconventional scaling of thermodynamic and transport quantities. Because of the vanishing density of states $\left[\varrho(E) \sim|E|^{1 / n}\right]$, this anisotropic semimetal (ASM) is stable against weak short-range interactions. However, for stronger interactions, the direct Dirac-semimetal to band-insulator transition can either (i) become a fluctuation-driven first-order transition (although unlikely in a particular microscopic model considered here, the anisotropic honeycomb lattice extended Hubbard model) or (ii) get avoided by an intervening broken-symmetry phase. We perform a controlled renormalization group analysis with the small parameter $\epsilon=1 / n$, augmented with a $1 / n$ expansion (parametrically suppressing quantum fluctuations in the higher dimension) by perturbing away from the one-dimensional limit, realized by setting $\epsilon=0$ and $n \rightarrow \infty$. We identify charge density wave (CDW), antiferromagnet (AFM), and singlet $s$-wave superconductivity as the three dominant candidates for broken symmetry. The onset of any such order at strong coupling $(\sim \epsilon)$ takes place through a continuous quantum phase transition across an interacting multicritical point, where the ordered phase, band insulator, Dirac, and anisotropic semimetals meet. We also present the phase diagram of an extended Hubbard model for the ASM, obtained via the controlled deformation of its counterpart in one dimension. The latter displays spin-charge separation and instabilities to CDW, spin density wave, and Luther-Emery liquid phases at arbitrarily weak coupling. The spin density wave and Luther-Emery liquid phases deform into pseudospin SU(2)-symmetric quantum critical points separating the ASM from the AFM and superconducting orders, respectively. Our phase diagram shows an intriguing interplay among CDW, AFM, and $s$-wave paired states that can be germane for a uniaxially strained optical honeycomb lattice for ultracold fermion atoms, or the organic compound $\alpha$-(BEDT-TTF $)_{2} \mathrm{I}_{3}$.

DOI: 10.1103/PhysRevX.8.011049

Subject Areas: Condensed Matter Physics, Strongly Correlated Materials

\section{INTRODUCTION}

A Dirac semimetal stands as a paradigmatic representative of a symmetry-protected gapless topological phase of matter that, for example, in two spatial dimensions, can be realized in pristine monolayer graphene [1-3]. In a planar system, such a phase can be envisioned as a bound state of an equal number of vortices and antivortices (with unit vorticity) in reciprocal space, such that the net vorticity is zero. The difference of the vorticities, coined as the "axial

Published by the American Physical Society under the terms of the Creative Commons Attribution 4.0 International license. Further distribution of this work must maintain attribution to the author(s) and the published article's title, journal citation, and DOI. vorticity" $\left(\mathcal{N}_{a}\right)$, is, however, finite and given by $\mathcal{N}_{a}=2 n$, where $n$ is an integer ( $n=1$ for graphene) that (modulo 2) in turn also defines the integer topological invariant/charge of a two-dimensional Dirac semimetal. Still, it is conceivable to tune some suitable band parameter to the drive system through a continuous topological quantum phase transition, where vortex and antivortex annihilate at a high symmetry point in the Brillouin zone, beyond which the system becomes a trivial band insulator. In the band insulator phase, $\mathcal{N}_{a}=0$. While a Dirac semimetal features linearly dispersing quasiparticles in all directions down to arbitrarily low energy, the critical fermions residing at the Dirac-semimetal to band-insulator quantum critical point (QCP) possess linear and quadratic dispersions along orthogonal directions in momentum space.

The quintessential properties of such a transition can be captured by a simple single-particle Hamiltonian, 


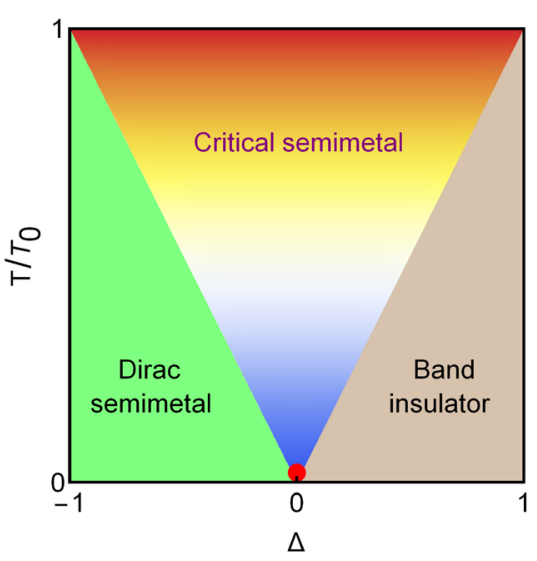

(a)

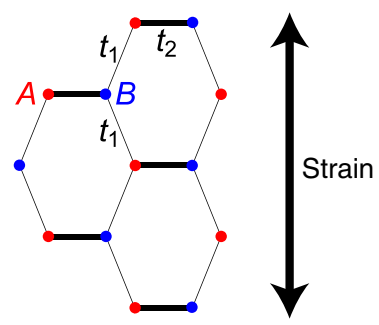

(b)

FIG. 1. (a) Schematic representation of the quantum critical fan at finite temperature associated with the direct transition between a two-dimensional Dirac semimetal and a symmetry-preserving band insulator, with $T_{0} \sim v^{2} /\left(k_{B} b\right)$. The signatures of the critical excitations, described by an anisotropic semimetal $[H(\mathbf{k}, 0)$ from Eq. (1)], get gradually washed out as the temperature is increased and lattice effects set in at high temperature $\left(T \sim T_{0}\right)$. The zero temperature quantum critical point in the noninteracting system, located at $\Delta=0$, is represented by the red dot. The critical regime can also be exposed by frequency and magnetic field (see text). The scaling of physical observables inside the critical fan, within the Dirac semimetal, or within the band insulator side of the transition is reported in Table I. (b) Hopping pattern in a uniaxially strained honeycomb lattice. Strong (weak) hopping amplitudes are represented by thick (thin) lines. The sites on the two sublattices are shown in red $(A)$ and blue $(B)$. The anisotropic semimetal is realized when $t_{2}=2 t_{1}$.

$$
H(\mathbf{k}, \Delta)=\sigma_{0}\left[v k_{x} \tau_{1}+\left(b k_{y}^{2}+\Delta\right) \tau_{2}\right],
$$

where two sets of Pauli matrices $\sigma_{\mu}=\left\{\sigma_{0}, \sigma_{1}, \sigma_{2}, \sigma_{3}\right\}$ and $\tau_{\mu}=\left\{\tau_{0}, \tau_{1}, \tau_{2}, \tau_{3}\right\}$, respectively, operate on the spin and orbital space (and $\sigma_{0}=\tau_{0}$ is the identity), and we set $\hbar=1$. Throughout this paper, we assume that spin is a good quantum number (neglecting weak spin-orbit coupling). In Eq. (1), $v$ and $b$ bear the dimensions of Fermi velocity and inverse mass, respectively, and $\Delta$ has the dimension of energy. The above Hamiltonian represents (i) a Dirac semimetal for $\Delta<0$ and (ii) a band insulator for $\Delta>0$, as shown in Fig. 1(a). In the Dirac semimetal phase, the Dirac points are located at $k_{x}=0, k_{y}= \pm \sqrt{-\Delta / b}$ [4].
TABLE I. Power-law scaling of various physical observables for the anisotropic semimetal (ASM) and Dirac semimetal (DSM). The Grüneisen ratio is defined as $\Gamma=\alpha / C_{P}$, where $\alpha$ is the thermal expansion parameter and $C_{P}$ is the specific heat, measured at constant pressure. The magnetic Grüneisen ratio is defined as $\Gamma_{H}=(\partial M / \partial T)_{H} / C_{H}$, where $M \propto H$ is the magnetization, $C_{H}$ is the molar specific heat, and $H$ is the magnetic field strength. When the Zeeman coupling dominates over the orbital one, $\Gamma_{H}$ has the same power-law dependence in the ASM and DSM (shown inside the parentheses in the fourth row). By contrast, when orbital coupling of the magnetic field dominates over Zeeman coupling, $\Gamma_{H}$ has distinct scaling behaviors in the ASM and DSM (displayed outside the parentheses in the fourth row). The explicit form of the dynamic optical conductivity $\left(\sigma_{j j}\right)$ and diamagnetic susceptibility $\left(\chi_{0}\right)$ in the ASM are, respectively, shown in Eqs. (3) and (4). The scaling of the diamagnetic susceptibility at finite temperature and low magnetic field (no Landau quantization) is shown in the last row. The crossover behavior between the ASM and the DSM at finite temperature, frequency, and magnetic field is discussed in the text. All physical observables display activated behavior in the band insulator.

\begin{tabular}{lcc}
\hline \hline Physical observable & ASM & DSM \\
\hline Compressibility $(\kappa)$ & $T^{1 / 2}$ & $T$ \\
Specific heat $\left(C_{v}\right)$ & $T^{3 / 2}$ & $T^{2}$ \\
Grüneisen ratio $(\Gamma)$ & $T^{-5 / 2}$ & $T^{-3}$ \\
Magnetic Grüneisen ratio $\left(\Gamma_{H}\right)$ & $T^{-3}\left(T^{-2}\right)$ & $T^{-4}\left(T^{-2}\right)$ \\
Dynamic conductivity $\left(\sigma_{x x, y y}\right)$ & $\omega^{-1 / 2}, \omega^{1 / 2}$ & $\omega^{0}$ (constant) \\
Diamagnetic susceptibility $\left(\chi_{0}\right)$ & $B^{-1 / 3}$ & $B^{-1 / 2}$ \\
Diamagnetic susceptibility $[\chi(T)]$ & $T^{-1 / 2}$ & $T^{-1}$ \\
\hline \hline
\end{tabular}

The Dirac-semimetal to band-insulator QCP is located at $\Delta=0$ [5], where the quasiparticle spectra, given by $E_{\mathbf{k}}= \pm \sqrt{v^{2} k_{x}^{2}+b^{2} k_{y}^{4}}$, display both linear (along $k_{x}$ ) and quadratic (along $k_{y}$ ) dependence on the two different components of momenta. The density of states of the anisotropic semimetal (ASM) vanishes as $\varrho(E) \sim \sqrt{E}$. We compute key thermodynamic and transport properties of the noninteracting ASM (see Table I).

In this paper, we study the effects of generic short-range electronic interactions on such an ASM. We assume that long-range Coulomb interactions [6,7] are screened, e.g., via a proximate gate. Because of the vanishing density of states, the ASM is stable against sufficiently weak shortrange interactions. By contrast, we show that the ASM can undergo a continuous quantum phase transition at strong interaction coupling through a multicritical point (tuned via interaction and anisotropy strengths) and enter into various broken-symmetry phases. At the multicritical points the Dirac semimetal, band insulator, ASM, and a brokensymmetry phase meet [see the blue dot in Fig. 2(b)]. We identify charge density wave (CDW), antiferromagnet (AFM), and spin-singlet $s$-wave superconductivity as 


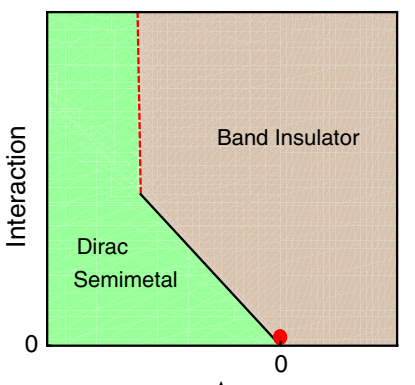

$\Delta$

(a)

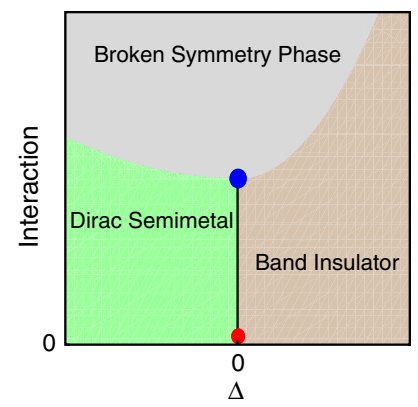

(b)
FIG. 2. Schematic phase diagrams of a two-dimensional Dirac material, residing in close proximity to the Dirac-semimetal to band-insulator quantum critical point (the red dot), in the presence of generic short-range interactions. Critical fermions possessing anisotropic dispersion are found along the black line in either subfigure. This direct transition can be avoided in two separate scenarios: (a) a strong-coupling scenario in which the critical anisotropic semimetal (ASM) is replaced by a line of firstorder transitions (red dashed line) and (b) an alternative scenario in which the ASM becomes unstable to the formation of a brokensymmetry phase. In this case, the blue dot represents a multicritical point separating the ASM from spontaneous ordering. Charge density wave, antiferromagnet (Néel), and $s$-wave superconductivity are the prominent candidates for the brokensymmetry phase [see also Fig. 4(b) and Fig. 12]. For weak coupling in (a), the only effect of the interactions is to shift the phase boundary separating the band insulator and Dirac semimetal. In (b), we assume that any such shift is compensated by the bare anisotropy $(\Delta)$. We do not here discuss instabilities of the Dirac semimetal (see text for discussion on this issue [8]).

leading candidates for the broken-symmetry phase. These conclusions obtain via the renormalization group (RG) controlled by an expansion about the one-dimensional limit, described below. We also consider an alternative scenario that could occur at strong coupling, wherein the Dirac semimetal can be separated from the band insulator by a fluctuation-driven first-order transition. These two possibilities are schematically displayed in Fig. 2. We do not discuss in this paper quantum phase transitions between the Dirac-semimetal and broken-symmetry phases. In addition to CDW, AFM, and $s$-wave pairing, a Dirac semimetal can accommodate additional fully gapped orders, such as a quantum anomalous/spin-Hall insulator or Kekule valence-bond solid [8]. Both of these arise because of the valley degree of freedom in the Dirac system, which is annihilated at the Dirac-semimetal to band-insulator (anisotropic semimetal) transition. Since the density of states in a Dirac semimetal (ASM) vanishes as $\varrho(E) \sim|E|(\sqrt{E})$, any ordering in a Dirac semimetal tuned close to the transition into the band insulator is expected to be preempted by those in the ASM, allowing us to focus solely on interaction effects in the latter.

In a half-filled honeycomb lattice, the ASM can be realized by applying uniaxial strain [9-12] [see Fig. 1(b)], which in real graphene would require an extremely large (and possibly unrealistic) distortion of the lattice. Nonetheless, in an optical honeycomb lattice for ultracold fermion atoms, the ASM can be achieved by tuning the depth of the laser trap, as has been reported in recent experiments [13-15]. In addition, the pressured organic compound $\alpha$-(BEDT-TTF $)_{2} \mathrm{I}_{3}$ [16-19], as well as black phosphorous (a system with a few layers of phosphorene) [20-22], may reside very close to the ASM QCP. A recent ARPES measurement is suggestive of anisotropic dispersion in black phosphorus [21]. Furthermore, the quasiparticles residing at the interface of $\mathrm{TiO}_{2} / \mathrm{VO}_{2}$ are also believed to possess such an anisotropic dispersion $[23,24]$. The QCP separating the Dirac semimetal and band insulator can also be accessed in uniaxially strained artificial/molecular graphene, as the hopping pattern in this system can be tuned quite efficiently [25].

A recent experiment has observed a first-order transition in $\alpha$-(BEDT-TTF $)_{2} \mathrm{I}_{3}$ [26], also found in our theoretical analysis. Additionally, a semimetal-Mott (most likely an AFM state) crossover in an optical honeycomb lattice driven by on-site repulsion has been reported in an experiment [14]. Therefore, our results are germane to a plethora of condensed matter systems, but also directly applicable for a strained optical honeycomb lattice (populated by neutral atoms). At the same time, our theoretical approach to the interacting ASM smoothly connects it to strong correlation physics in one dimension and might serve as a platform to explore how 1D physics such as spin-charge separation and fractionalization get modified in higher dimensions. We now highlight the central results of our study.

\section{A. Noninteracting system}

We begin by noting some hallmark signatures of the noninteracting ASM, the scaling behavior of thermodynamic and transport quantities that can directly be observed in experiments. Even though the ASM can only be found at $\Delta=0$ when $T=0$, its imprint can be realized over the wide quantum critical regime shown in Fig. 1(a) at finite temperature [up to a temperature $T_{0} \sim v^{2} /\left(k_{B} b\right)$, beyond which details of the lattice become important]. The powerlaw scaling of various thermodynamic quantities, shown in Table I (center column), is essentially governed by the scaling of density of states, which, in the ASM, vanishes as $\varrho(E) \sim \sqrt{E}$. Consequently, the compressibility scales as $\kappa \sim \sqrt{T}$, and the specific heat vanishes as $C_{v} \sim T^{3 / 2}$ (at charge neutrality or for $T \gg|\mu|$, where $\mu$ denotes the chemical potential). If $\Delta<0$, the scaling of thermodynamic quantities displays a smooth crossover from the ones in an ASM to those in a Dirac semimetal (displayed in the right column of Table I) around a crossover temperature $T_{*} \sim|\Delta| / k_{B}$ [27]. On the other hand, for $\Delta>0$, the thermodynamic quantities display activated scaling for $T<T_{*}$. 


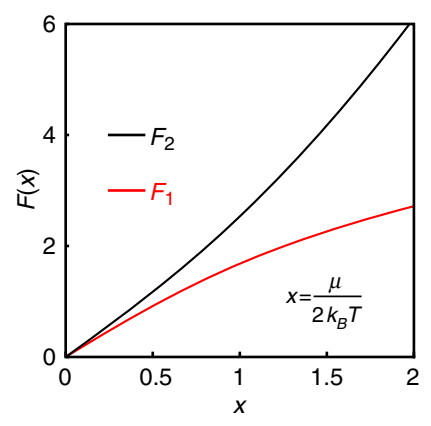

(a)

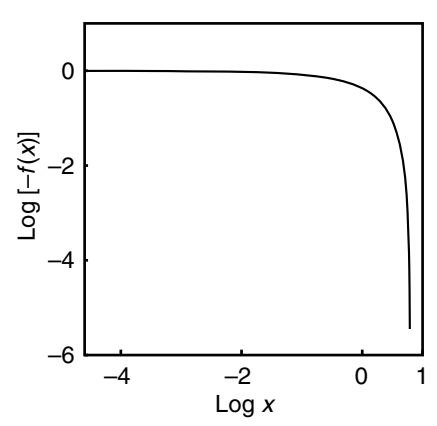

(b)
FIG. 3. (a) Scaling of two functions appearing in the expression for the Drude conductivity of the anisotropic semimetal in the $x$ (given by $F_{1}$ ) and $y$ (given by $F_{2}$ ) directions [see Eq. (2)] versus the dimensionless argument $X=\mu /\left(2 k_{B} T\right)$. Here $\mu$ is the chemical potential and $T$ is the temperature. (b) Scaling of the function $f(x)$ versus the dimensionless argument $x$ (the ratio of thermal de Broglie wavelength to magnetic length) that controls the diamagnetic susceptibility of an anisotropic semimetal at finite temperature [see Eq. (5)].

The quantum critical regime shown in Fig. 1(a) can also be exposed by frequency $(\omega)$ as long as $\omega \lesssim \omega_{0} \sim v^{2} / b$ and $\omega>|\Delta|$. For example, the Drude conductivity for noninteracting electrons in the ASM scales as

$$
\left.\sigma_{j j}(\omega)\right|_{\omega \rightarrow 0}=\frac{a_{j} e^{2}}{h}\left[\frac{T_{0}}{T}\right]^{\frac{3}{2}-j} F_{j}\left(\frac{\mu}{2 k_{B} T}\right) \delta\left(\frac{\omega}{k_{B} T}\right),
$$

while the interband component of the optical conductivity goes as

$$
\sigma_{j j}(\omega, T)=\frac{a_{j} e^{2}}{2 h}\left[\frac{\omega_{0}}{\omega}\right]^{\frac{3}{2}-j} \sum_{\alpha= \pm} \tanh \left[\frac{\omega+2 \alpha \mu}{4 k_{B} T}\right],
$$

where $a_{x} \approx 0.06262, a_{y} \approx 0.20602$, and $k_{B} T_{0}=\omega_{0}=v^{2} / b$. In the last two equations, $j=1,2$, respectively, corresponds to $x$ and $y$. The scaling of the two universal functions $F_{1}(x)$ and $F_{2}(x)$ is shown in Fig. 3(a), and the detailed calculation of the optical conductivity is presented in Appendix A. Note that, as frequency is lowered, the interband component of the optical conductivity displays a smooth crossover from the scaling highlighted in Eq. (3) to the one for massless Dirac fermions $\left(\sigma_{x x, y y} \sim e^{2} / h\right.$ without any leading power-law dependence on $\omega$ ) for $\omega<|\Delta|$ and $\Delta<0$. By contrast, the optical conductivity displays activated behavior at low frequency $(\omega<\Delta)$ when $\Delta>0$.

The diamagnetic susceptibility of the ASM at $T=0$ is given by

$$
\chi_{0}=-\mathcal{A}(e / h)(e v \sqrt{b / 2})^{2 / 3} B^{-1 / 3},
$$

which diverges as $B^{-1 / 3}$, where $\mathcal{A} \approx 0.075$. A detailed calculation is presented in Appendix B. By contrast, the diamagnetic susceptibility of a two-dimensional Dirac semimetal diverges as $\chi_{0} \sim B^{-1 / 2}$ [28], while that for three-dimensional Dirac [29] and Weyl [30] semimetals scales as $\chi_{0} \sim \log \left(B / B_{0}\right)$, where $B_{0} \sim 1 / a^{2}$ and $a$ is the lattice spacing. The power-law dependence of the diamagnetic susceptibility, shown in Eq. (4), can be observed in experiments only when $B>\Delta^{2}$, so that we expose the critical regime associated with the noninteracting QCP [Fig. 1(a)] by an external magnetic field. [31] Otherwise, with decreasing strength of the magnetic field the diamagnetic susceptibility displays a smooth crossover from $B^{-1 / 3}$ to $B^{-1 / 2}$ scaling when $B<\Delta^{2}$ and $\Delta<0$.

At finite temperature, the diamagnetic susceptibility assumes the following universal scaling form (see Appendix B for details),

$$
\chi_{T}=\chi_{0}\left[1+f\left(\lambda_{t h} / \ell_{B}\right)\right],
$$

where $\lambda_{T h}=\hbar \sqrt{v}(b / 2)^{1 / 4} /\left(k_{B} T\right)^{3 / 4}$ is the thermal de Broglie wavelength for the critical fermions [32]. The scaling of the universal function $f(x)$ is shown in Fig. 3(b). Our proposed scaling at finite temperature $\left(\chi_{T}\right)$ is valid as long as $\ell_{B}<\lambda_{T h}<\xi_{\Delta} \sim 1 / \Delta$ [33].

\section{B. Electron-electron interactions}

Next we turn to the effects of generic short-range electronic interactions on the ASM. We focus on the particle-hole symmetric system at charge neutrality with $\mu=0$, although our results will also strongly influence the finite temperature physics when $T \gg|\mu|$. Scaling of the density of states yields a negative scaling dimension for any four-fermion interaction coupling $g$, namely, $[g]=-1 / 2$. Consequently, the noninteracting ASM QCP separating the Dirac semimetal and the band insulator remains stable against sufficiently weak shortrange interactions. However, at stronger interaction coupling, we predict that one of two possible scenarios occurs: Either (i) the Dirac-semimetal to band-insulator transition becomes a fluctuation-driven first-order transition (see Sec. VA) or (ii) the direct transition gets masked by an intervening broken-symmetry phase (see Sec. VI). These two scenarios are schematically depicted in Figs. 2(a) and 2(b), respectively. Demonstrating the first-order transition requires a nonperturbative approach, which we here implement using large- $N$ mean field theory (where $N$ is the number of flavors of critical fermions; $N=2$ for spin-1/2 electrons in the strained honeycomb model). The onset of broken symmetry takes place through a continuous quantum phase transition. The nucleation of broken symmetry through a continuous transition at intermediate coupling can be established by a RG analysis.

To control the RG calculation, we deform the Hamiltonian from Eq. (1) to 
$H(\mathbf{k}, \Delta) \rightarrow H_{n}(\mathbf{k}, \Delta)=\sigma_{0}\left[v k_{x} \tau_{1}+\left(b k_{y}^{n}+\Delta\right) \tau_{2}\right]$,

where $n$ is an even integer, so that this transformation does not alter the symmetry of the system (discussed in Sec. II B). The density of states in the deformed system scales as $\varrho(E) \sim E^{1 / n}$ and, consequently, the scaling dimension for any four-fermion interaction coupling $g$ becomes $[g]=-1 / n$. In the limit $n \rightarrow \infty$, the density of states becomes finite. With purely local interactions, this limit corresponds to a decoupled collection of one-dimensional systems composed of massless Dirac fermions with spectra $\varepsilon_{\mathbf{k}}= \pm v k_{x}$. All local interactions have vanishing engineering dimension in this one-dimensional limit. Hence, $n \rightarrow \infty$ sets the marginality condition for generic short-range interactions in the ASM, and the perturbative RG calculation can be controlled in terms of a small parameter $\epsilon=1 / n$, following the spirit of the $\epsilon$ expansion [34] with $[g]=-\epsilon$. Thus, our RG calculation is performed in an effective dimension $d_{*}=1+\epsilon$ and, precisely at $\epsilon=0$, local four-fermion interactions are marginal.

The above construction allows us to further control quantum fluctuations arising from the finite quasiparticle dispersion along the $k_{y}$ direction by a parameter $1 / n$, entering through loop corrections in the perturbative RG calculation. Thus, our RG analysis is simultaneously controlled by two small parameters, $\epsilon$ (which sets the engineering dimension of the interaction coupling $g$ ) and $1 / n$ (which controls the quantum fluctuations). In this framework, the RG flow equations take the form

$$
\frac{d g_{\mu}}{d l} \equiv \beta_{g_{\mu}}=-\epsilon g_{\mu}+\sum_{\mu, \nu} A_{\mu, \nu} g_{\mu} g_{\nu}+\frac{1}{n} \sum_{\mu, \nu} B_{\mu, \nu} g_{\mu} g_{\nu},
$$

where $l$ is the logarithm of the RG length scale, and the summation over $\mu, \nu$ runs over all symmetry-allowed, linearly independent coupling constants $\left\{g_{\mu}\right\}$. To leading order, $A_{\mu, \nu}$ and $B_{\mu, \nu}$ are numerical ( $n$-independent) matrices that we compute.

We emphasize that we treat $\epsilon$ and $1 / n$ as independent parameters in our RG scheme, although we should set these equal at the end. There are physical and technical reasons for this. Physically, $\epsilon$ enters as the engineering dimension for interaction couplings $\left[g_{\mu}\right]=-\epsilon$ [Eq. (7)]. This is due entirely to dimensional analysis. While $\epsilon>0$ makes all such interactions irrelevant at weak coupling, it preserves key aspects of the physics special to one dimension, such as spin-charge separation. By contrast, the explicit $1 / n$ corrections in Eq. (7) obtain from loop integrations involving the $k_{y}$ dispersion and, thus, encode quantum fluctuations beyond 1D. The Hubbard model phase diagram in Fig. 4(b) (discussed below) obtains only after quantum corrections are included, since these select between competing orders that are degenerate when $\epsilon>0$ but $1 / n=0$ (see Table $\mathrm{V}$ and Sec. VIII for details).
Technically, our calculation works like an $\epsilon$ expansion, [35] since we expand order by order in the interaction strengths. The expansion of the one-loop corrections (see Appendix E) in $1 / n$ is not technically required, but is performed for physical clarity [36].

As a benchmark, we will show that if we set $\epsilon=0$ and send $n \rightarrow \infty$ in Eq. (7), we recover well-established results for interacting fermions in one dimension. For example, (i) the $\beta$-function vanishes for spinless fermions (described by a two-component Dirac fermion and a single Luttinger current-current interaction coupling $g$ ), and (ii) the above set of flow equations displays spin-charge separation, with independent charge and spin KosterlitzThouless transitions for spin-1/2 electrons $[42,43]$.

The central outcome of our analysis can be summarized in the form of a phase diagram for an extended Hubbard model appropriate for uniaxially strained graphene, tuned to the Dirac-semimetal to band-insulator QCP. The interactions are defined via

$$
H_{\mathrm{UV}}=\frac{U}{2} \sum_{\vec{X}=\vec{A}, \vec{B}} n_{\uparrow}(\vec{X}) n_{\downarrow}(\vec{X})+\frac{V}{2} \sum_{\vec{A}, i, \sigma=\uparrow, \downarrow} n_{\sigma}(\vec{A}) n_{\sigma}\left(\vec{A}+\vec{b}_{i}\right) .
$$

Here, $U$ and $V$, respectively, denote the on-site and nearestneighbor (NN) interaction strengths, and $n_{\sigma}(\vec{X})$ is the fermion number operator at position $\vec{r}=\vec{X}$, with spin projection $\sigma=\uparrow, \downarrow$. For the sake of simplicity, we assume that the strength of NN repulsion is the same among all three NN sites in uniaxially strained graphene. [47] The extended Hubbard model in one dimension has been extensively studied by analytical [42,43] and numerical [38-41] methods (see also Sec. VIII A). The phase diagrams for one- and two-dimensional systems are, respectively, shown in Figs. 4(a) and 4(b). A detailed analysis of the extended Hubbard model is presented in Sec. VIII.

The relevance of Eq. (8) and Fig. 4(b) to experiments is as follows. A strained optical honeycomb lattice for ultracold atoms [13-15] constitutes an ideal platform to realize the entire $V=0$ axis of the phase diagram, as the strength of repulsive and attractive Hubbard interaction can be tuned quite efficiently, while NN interactions are negligible [48].

In pristine (unstrained) graphene $U \sim 9 \mathrm{eV}$, while $V \sim$ $5 \mathrm{eV}$ for $\kappa=2.5$, where $\kappa$ is the effective dielectric constant of the medium [49]; the hopping strength $t \sim 2.8 \mathrm{eV}$. When uniaxially strained, the strength of on-site repulsion in graphene is likely unchanged, while the NN interaction gets slightly weaker, thus allowing access to the onsiterepulsion-dominated regime of the phase diagram. A very large strain of order $20 \%$ is required to tune to the Diracsemimetal to band-insulator transition point [11]. A strain around $13 \%$ was recently achieved using MEMS (albeit in three-layer graphene [50]), corresponding to $t_{2} \sim 1.5 t_{1}$ [11] 


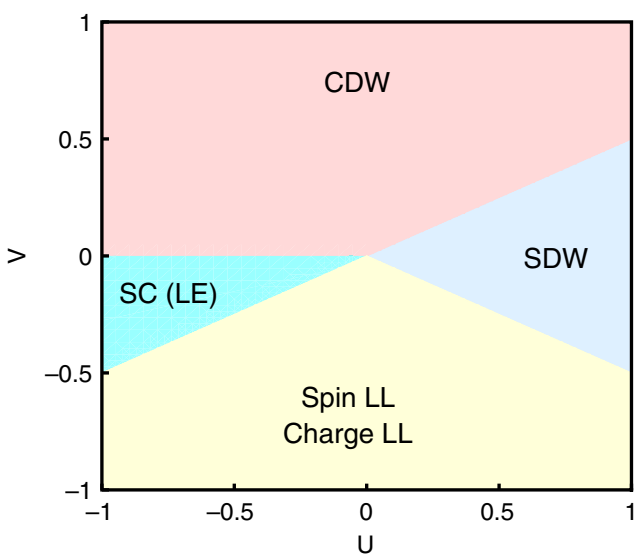

(a)

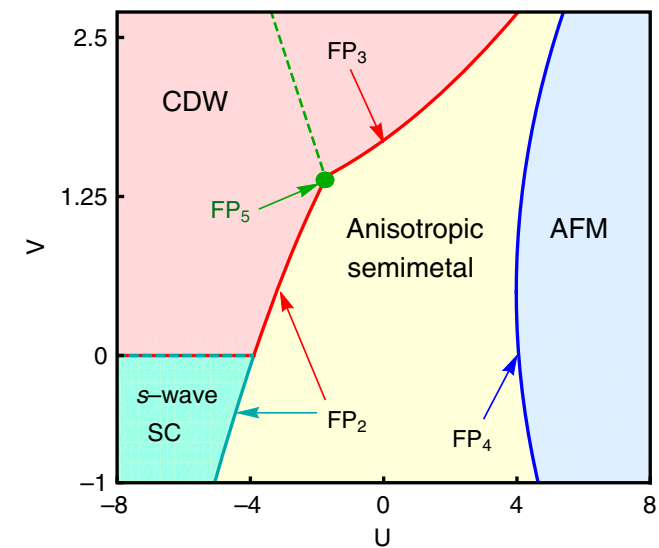

(b)

FIG. 4. The phase diagram of the extended Hubbard model for spin-1/2 electrons in (a) one dimension and (b) the two-dimensional anisotropic semimetal (ASM), obtained in this work. Here, $U$ and $V$, respectively, correspond to on-site and nearest-neighbor interactions; see Eq. (8). The 1D extended Hubbard model has been extensively studied; see, e.g., [38-41]. Here, we limit attention to the well-known predictions that obtain from bosonization [42,43], expected to hold for sufficiently weak coupling. In 1D, electronic quasiparticles are ill defined for arbitrarily weak interactions. The interacting phases are shown in panel (a) and consist of (i) spin density wave (SDW), (ii) CDW, (iii) Luther-Emery liquid (LE), and (iv) coexisting spin and charge Luttinger liquids (LLs). In the continuum limit, these phases exhibit complete spin-charge separation [43]; the charge is gapped in the CDW and SDW phases, while the spin is gapped in the CDW and LE phases. The latter is a 1D precursor to superconductivity. We obtain a qualitatively similar phase diagram for the ASM, shown in (b). The key differences are that the quasi-long-range-ordered SDW and LE phases are, respectively, replaced with true long-range-ordered AFM and $s$-wave superconductivity (SC) (at zero temperature), and that the CDW, AFM, and SC orders appear at finite coupling as a result of the vanishing density of states. In (b), we set $n=10$ [Eq. (6)] to parametrically suppress higher-dimensional quantum fluctuations (see Sec. V for a detailed discussion); here $U$ and $V$ are dimensionless couplings measured in units of $\epsilon$. The phase diagram (b) is obtained by simultaneously solving the RG flow equations for four-fermion coupling constants and order parameter source terms (see Sec. VII). For $V=0$ and sufficiently attractive $U<0$, simultaneous nucleation of CDW and $s$-wave pairing occurs (dashed red/cyan line). Along this line, pseudospin SU(2) symmetry [44-46] gets spontaneously broken. Various quantum critical fixed points $\mathrm{FP}_{j}$ (see Table IV) control different segments of the phase boundaries, indicated in panel (b). Fixed points $\mathrm{FP}_{2,4}$ exhibit emergent pseudospin $\mathrm{SU}(2)$ symmetry, while this is broken at $\mathrm{FP}_{3}$. In the CDW phase (red region) in panel (b), the dashed green line is a crossover boundary. Above the line of coexistence with the CDW, the $s$-wave superconductivity exhibits short-range ordering that smoothly vanishes at the dotted green line. This is governed by the bicritical point $\mathrm{FP}_{5}$ (see Table IV), where the ASM-CDW phase boundary displays a kink (green dot).

[see Fig. 1(b)]. This is still relatively far from the transition at $t_{2}=2 t_{1}$, but even larger values of strain may be possible. As emphasized above, it is not necessary to tune precisely to the transition point to potentially realize the Hubbard phase diagram in Fig. 4(b), since transitions out of the ASM are expected to dominate over those out of the Dirac semimetal because of the larger density of states of the former. The parabolic curvature responsible for the density of states enhancement turns on at intermediate single-particle energies, even when there is still some splitting between degenerate Dirac semimetal valleys at zero energy [see the wide quantum critical regime for ASM in Fig. 1(a)], and intermediate energies are more relevant for $U, V>t$.

Another method to achieve the ASM in graphene could exploit a moiré pattern. Such a pattern can occur in twisted bilayers [51], where it generates satellite Dirac points in the vicinity of the $K$ and $K^{\prime}$ points. A smaller (but still substantial) strain could be used to further merge two satellites along a high-symmetry direction.

The strengths of $U$ and $V$ in black phosphorus, at a $\mathrm{TiO}_{2} / \mathrm{VO}_{2}$ interface, and in $\alpha$-(BEDT-TTF $)_{2} \mathrm{I}_{3}$ are not precisely known. Nonetheless, by changing the substrate on which these quasi-two-dimensional systems reside, one can efficiently tune the strength of NN repulsion, with suspended samples endowed with strongest NN repulsion. Furthermore, by changing the distance $(\xi)$ between a gate and the sample, one can also tune the strength of $\mathrm{NN}$ repulsion [52]. The screened Coulomb interaction is

$$
\begin{aligned}
V(r) & \sim U_{0} \xi\left[1 / r-1 / \sqrt{r^{2}+\xi^{2}}\right] \\
& \sim U_{0} \sqrt{\xi / r} e^{-\pi r / \xi}
\end{aligned}
$$

where the first (second) result applies to a single-gated (double-gated) sample. In this equation, $U_{0} \sim e^{2} /(\kappa \xi)$; the nearest-neighbor repulsion is $V=V(a)$, with $a$ the lattice spacing. Thus, different gate configurations can tune the relative strength of $U$ and $V$ and experimentally access various repulsive regimes and quantum phase transitions of the phase diagram in Fig. 4(b). Black phosphorous (already residing close to the ASM fixed point), in particular, can be 
tuned to the ASM band structure via impurity doping and the giant Stark effect [21], but this also shifts the chemical potential away from charge neutrality. The effects of finite chemical potential on the phase diagram shown in Fig. 4(b) are an important avenue for future work. We hope that the above discussion will motivate first-principle calculations in other materials (similar to Ref. [49]) to estimate interaction strengths in various $2 \mathrm{D} \mathrm{ASMs}$ and future experiments to search for various broken-symmetry phases.

Superconductivity in an ASM can also be induced via a proximity effect. Proximity-induced superconductivity has been recently achieved in monolayer graphene, when deposited on rhenium (an $s$-wave superconductor), and $\mathrm{Pr}_{2-x} \mathrm{Ce}_{x} \mathrm{CuO}_{4}$ (a $d$-wave superconductor, with coherence length $\xi_{S} \sim 30 \mathrm{~nm}$ ), with respective superconducting transition temperatures $T_{c} \sim 2 \mathrm{~K}$ [53] and $T_{c} \sim 4.2 \mathrm{~K}$ [54]. Therefore, it is conceivable to induce superconductivity in an ASM by controlling the distance $(\xi)$ between strained graphene or black phosphorus and bulk superconducting materials (such as $\mathrm{Rh}, \mathrm{Pr}_{2-x} \mathrm{Ce}_{x} \mathrm{CuO}_{4}$ ).

We now discuss salient features of the two phase diagrams shown in Figs. 4(a) and 4(b). In 1D, electronic quasiparticles are ill defined for arbitrarily weak interactions. The 1D extended Hubbard phase diagram reviewed in Fig. 4(a) is that obtained via bosonization, which predicts spin-charge separation $[42,43]$. The spin and charge sectors can each separately exhibit gapless Luttinger liquid or gapped Mott insulating phases. This leads to the four composite phases shown in Fig. 4(a): (i) spin density wave (SDW), (ii) CDW, (iii) Luther-Emery liquid (LE), and (iv) coexisting spin and charge Luttinger liquids. The charge (spin) is gapped in the CDW and SDW (CDW and LE) phases. The SDW and Luther-Emery phases exhibit quasi-long-range order in the spin and charge sectors, respectively; these are precursors to antiferromagnetism and superconductivity in higher dimensions. The CDW phase exhibits true long-range, sublattice-staggered charge order at zero temperature.

The phase diagram of the extended Hubbard model in the two-dimensional ASM is shown in Fig. 4(b), as obtained via our RG analysis. The 2D ASM (yellow shaded region) remains stable against sufficiently weak local interactions. The onset of ordered phases takes place at a finite strength coupling $\left(g_{\mu} \sim \epsilon\right)$ in the ASM. In terms of the interacting phases, 1D [Fig. 4(a)] and 2D [Fig. 4(b)] are qualitatively similar. The key difference is that the quasilong-range-ordered SDW and LE phases in 1D are, respectively, replaced with true long-range-ordered Néel AFM and $s$-wave SC in 2D (at zero temperature).

Sufficiently strong repulsive $U>0$ drives the ASM through a continuous quantum phase transition and places it into the Néel AFM phase, which breaks spin SU(2) and time-reversal symmetry on the honeycomb lattice. Strong on-site attraction $U<0$ with $V=0$ induces the simultaneous nucleation of $s$-wave pairing and CDW orders, stemming from the spontaneous breaking of the exact pseudospin $\mathrm{SU}(2)$ symmetry [55]. Nonzero $V$ breaks the pseudospin symmetry, preferring CDW ( $s$-wave superconductivity) for repulsive $V>0$ (attractive $V<0$ ) coupling. The CDW and AFM phases, respectively, exhibit sublattice-staggered charge and collinear spin orders; they preserve translational invariance on the bipartite honeycomb lattice, but break reflection invariance across any line of bonds.

Thus, the phase diagram of the extended Hubbard model in the ASM can be considered a controlled deformation (by two small parameters $\epsilon$ and $1 / n$ ) of its counterpart in one spatial dimension. In addition, the results obtained from our leading-order $\mathrm{RG}$ analysis are consistent with pseudospin $\mathrm{SU}(2)$ symmetry [44-46] for $V=0$. The fixed points $\mathrm{FP}_{2}$ and $\mathrm{FP}_{4}$ that govern the transitions along this line exhibit degenerate scaling dimensions for all operators residing in each irreducible representation of this symmetry (see Table V and Fig. 11). The proposed phase diagram in Fig. 4(b) should therefore qualitatively describe the strongly interacting ASM, residing at the phase boundary between the Dirac semimetal and band insulator, for which $\epsilon=\frac{1}{2}$ and $n=2$.

Approaching from within the $s$-wave SC and AFM broken-symmetry phases, the pairing amplitude and Néel order parameters vanish continuously at the quantum critical points $\mathrm{FP}_{2}$ and $\mathrm{FP}_{4}$, respectively. These transitions can, therefore, be regarded as the two-dimensional deformations of the 1D quasi-long-range-ordered LE and SDW phases. To the leading order in the $\epsilon$ and $1 / n$ expansion, the correlation length exponent $\nu^{-1}=\epsilon$ is the same across all continuous transitions to broken-symmetry phases [56]. This is, however, an artifact of the leading-order calculation. Setting $\epsilon=1 / 2$, the transition or crossover temperature $\left(T_{c}\right)$ between broken-symmetry phases and the ASM scales as $T_{c} \sim \delta^{2}$, while that to the Dirac semimetal scales as $T_{c} \sim \delta$, where $\delta=\left(X-X_{c}\right) / X_{c}$ is the reduced control parameter for the zero-temperature quantum phase transition from ASM or Dirac semimetal into the ordered phase, with $X=U, V$ [Eq. (8)], for example, and $X_{c}$ as the critical strength for ordering. Thus, even though the ASM lives adjacent to a Dirac semimetal, the scaling of transition/crossover temperature to these scale distinctly. The quantum phase transition in the former system should precede the one in the Dirac semimetal if the latter is tuned close to the transition into the band insulator, since the ASM density of states vanishes more slowly with energy.

Each of the interacting QCPs (discussed in Sec. VI) controlling various continuous transitions into brokensymmetry phases are expected to accommodate strongly interacting non-Fermi liquids that lack sharp quasiparticle excitations. It would be extremely interesting to look for remnants of spin-charge separation in the two-loop selfenergy, which will give an anomalous dimension and lifetime to the fermion field. Our proposed phase diagram 
and associated quantum critical phenomena can be directly tested by quantum Monte Carlo simulations on the honeycomb lattice with on-site $(U)$ and nearest-neighbor $(V)$ interaction, as the extended Hubbard model can now be simulated without encountering the infamous sign problem [57-64]. The phase diagram (or at least some part of it) can also be exposed in the future in optical honeycomb lattice experiments with ultracold fermion atoms, wherein the strength of interactions can possibly be efficiently tuned. Our methodology can also be adopted to investigate the effects of electronic interactions in various other itinerant systems, possessing anisotropic fermionic dispersion, such as general Weyl semimetals [65], which we discuss qualitatively in Sec. IX.

\section{Outline}

The rest of the paper is organized as follows. In Sec. II we establish the field theory description of the noninteracting Dirac-semimetal to band-insulator quantum phase transition and discuss its symmetries. Possible brokensymmetry phases (both excitonic and superconducting) and the associated quasiparticle spectra are discussed in Sec. III. In Sec. IV, we describe the minimal models for spinless and spin-1/2 versions of the interacting ASM. In Sec. V, we determine the effects of electronic interactions on the ASM for spinless fermions. The RG analysis for spin- $1 / 2$ fermions, connection to 1D spin-charge separation, existence of various interacting QCPs, and associated quantum critical phenomena are discussed in Sec. VI. In Sec. VII, we determine the nature of the broken-symmetry phases at strong coupling across various continuous transitions for the interacting spin-1/2 model. Section VIII provides a more detailed discussion of the extended Hubbard model [Eq. (8)]. We summarize our findings, discuss applications of our methodology in other correlated systems, and highlight prospects for future work in Sec. IX. Additional technical details are relegated to the appendixes.

\section{NONINTERACTING SYSTEM: FIELD THEORY AND SYMMETRIES}

In this section, we construct the field theoretic description of the QCP separating a two-dimensional Dirac semimetal and a band insulator in a noninteracting system, and we discuss the symmetries of the ASM separating these two phases.

\section{A. Hamiltonian, Lagrangian, and scaling}

The universality class of the Dirac-semimetal to bandinsulator quantum phase transition is captured by the quadratic Hamiltonian from Eq. (1) for $\Delta=0$. The four-component spinor basis can be chosen as $\Psi^{\top}=$ $\left[c_{A, \mathbf{k}, \uparrow}, c_{B, \mathbf{k}, \uparrow}, c_{A, \mathbf{k}, \downarrow}, c_{B, \mathbf{k}, \downarrow}\right]$, where $c_{X, \mathbf{k}, \sigma}$ is the fermion annihilation operator with momentum $\mathbf{k}$, spin projection $\sigma=\uparrow, \downarrow$, and on sublattice $X=A / B$ of the honeycomb lattice. In other compounds, such as black phosphorus or $\alpha$-(BEDT-TTF $)_{2} I_{3}, X$ represents orbital degrees of freedom.

The imaginary time $(t)$ action associated with the noninteracting Hamiltonian reads

$$
\mathcal{S}_{0}=\int d^{2} \mathbf{r} d t^{\dagger}(\mathbf{r}, t) \mathcal{L}_{0} \Psi(\mathbf{r}, t),
$$

where $\mathbf{r} \equiv(x, y)$, and the kinetic operator $\mathcal{L}_{0}$ is given by

$$
\mathcal{L}_{0}=\partial_{t}+H_{n}(\mathbf{k} \rightarrow-i \nabla, \Delta) .
$$

Here, we define $\mathcal{L}_{0}$ via the deformation $H(\mathbf{k}, \Delta) \rightarrow$ $H_{n}(\mathbf{k}, \Delta)$ [see Eq. (6)], which leaves all the symmetries of the Hamiltonian unaffected (since $n$ is here an even integer).

We now introduce the notion of coarse-graining for this model, under which we send $t \rightarrow e^{l} t, x \rightarrow e^{l} x$, and $y \rightarrow e^{l / n} y$; the field transforms as $\Psi \rightarrow e^{-l(1+1 / n) / 2} \Psi$. Here, $l$ denotes the logarithm of the renormalization group length scale. The imaginary time action $\mathcal{S}_{0}$ remains invariant, so that the Fermi velocity $(v)$ and inverse mass $(b)$ are effectively dimensionless (carry zero scaling dimension $[v]=[b]=0$ ). On the other hand, the scaling dimension of $\Delta$ is $[\Delta]=1$. This is a relevant perturbation at the Diracsemimetal to band-insulator QCP that controls the transition between these two phases that possess the same symmetry. The scaling dimension of $\Delta$ yields the correlation length exponent for this noninteracting QCP: $\nu^{-1}=1$.

\section{B. Symmetries}

The Hamiltonian describing the ASM possesses a discrete chiral symmetry [also known as sublattice symmetry (SLS)], generated by a unitary operator $\sigma_{0} \tau_{3}$ and encoded in the condition

$$
-\sigma_{0} \tau_{3} H_{n}(\mathbf{k}, \Delta) \sigma_{0} \tau_{3}=H_{n}(\mathbf{k}, \Delta)
$$

Time-reversal symmetry is generated by the antiunitary operator $\mathcal{T}=\sigma_{2} \tau_{3} K$, where $K$ is complex conjugation, and $\mathcal{T}^{2}=-1$. The Hamiltonian $H_{n}(\mathbf{k}, \Delta)$ also possesses spin $\mathrm{SU}(2)$ symmetry generated by $\vec{S}=\vec{\sigma} \tau_{0}$, and $x$-reflection symmetry defined via

$$
\sigma_{0} \tau_{2} H_{n}\left(-k_{x}, k_{y}, \Delta\right) \sigma_{0} \tau_{2}=H_{n}\left(k_{x}, k_{y}, \Delta\right) .
$$

This symmetry operation encodes invariance of the system under the exchange of two sublattices or orbitals and will be denoted by $\mathcal{R}_{\pi}$ (see Table II) $[66,67]$. We relegate a detailed discussion on the symmetry properties of the ASM to Appendix C. In the next section, we discuss various possible ordered phases that break at least one of the above symmetries of the noninteracting system. 
TABLE II. All possible local (momentum-independent or intra-unit-cell) order parameters in the particle-hole or excitonic channel (first eight rows) and particle-particle or superconducting channel (last four rows) and their transformation properties under various discrete and continuous symmetry operations, discussed in Sec. II B. Under spin SU(2), bilinears transform in either the singlet (0) or triplet (1) representations. Note that the anisotropy parameter (AP) preserves all microscopic symmetries and, respectively, gives rise to gapless and gapped spectra for $\Delta<0$ and $\Delta>0$ on the corresponding sides of the Dirac-semimetal to band-insulator transition. The second to last column shows whether the quasiparticle spectrum inside a given broken-symmetry phase is fully gapped or not. Notice that only three ordered phases, namely, the CDW, Néel antiferromagnet (AFM), and $s$-wave SC, yield a fully gapped spectrum. These three orders are energetically most favored, at least within the framework of the weak coupling renormalization group analysis [see, for example, the phase diagram of the extended Hubbard model in Fig. 4(b)]. The last column labels the corresponding order-parameter (OP) source term (see also Sec. VII). The density, $x$ current, and $x$ spin current are included here for completeness, although these are not true order parameters. We note that, because of the lack of valley degrees of freedom, a two-dimensional anisotropic semimetal does not allow any topological order such as a quantum anomalous/spin Hall insulator, which is odd under the exchange of two valleys, unlike the situation in a Dirac semimetal [8].

\begin{tabular}{|c|c|c|c|c|c|c|c|}
\hline Bilinear & Description & SLS & $\mathcal{T}$ & $\mathcal{R}_{\pi}$ & Spin SU(2) & Gap? & $\mathrm{OP}$ \\
\hline$\Psi^{\dagger} \sigma_{0} \tau_{0} \Psi$ & Density & $x$ & $\checkmark$ & $\checkmark$ & 0 & No & $\Delta_{0}^{s}$ \\
\hline$\Psi^{\dagger} \sigma_{0} \tau_{1} \Psi$ & $x$ current & $\checkmark$ & $x$ & $x$ & 0 & No & $\Delta_{1}^{s}$ \\
\hline$\Psi^{\dagger} \sigma_{0} \tau_{2} \Psi$ & $\mathrm{AP}$ & $\checkmark$ & $\checkmark$ & $\checkmark$ & 0 & Yes/no & $\Delta_{2}^{s}$ \\
\hline$\Psi^{\dagger} \sigma_{0} \tau_{3} \Psi$ & CDW & $x$ & $\checkmark$ & $x$ & 0 & Yes & $\Delta_{3}^{s}$ \\
\hline$\Psi^{\dagger} \vec{\sigma} \tau_{0} \Psi$ & Ferromagnet & $x$ & $x$ & $\checkmark$ & 1 & No & $\Delta_{0}^{t}$ \\
\hline$\Psi^{\dagger} \vec{\sigma} \tau_{1} \Psi$ & $x$ spin current & $\checkmark$ & $\checkmark$ & $x$ & 1 & No & $\Delta_{1}^{t}$ \\
\hline$\Psi \vec{\sigma} \tau_{2} \Psi$ & Spin BDW & $\checkmark$ & $x$ & $\checkmark$ & 1 & No & $\Delta_{2}^{t}$ \\
\hline$\Psi^{\dagger} \vec{\sigma} \tau_{3} \Psi$ & AFM & $x$ & $\times$ & $x$ & 1 & Yes & $\Delta_{3}^{t}$ \\
\hline$\Psi \sigma_{2} \tau_{3} \Psi$ & $s$-wave SC & $\checkmark$ & $\checkmark$ & $\checkmark$ & 0 & Yes & $\Delta_{s}$ \\
\hline$\Psi \sigma_{2} \tau_{1} \Psi$ & Chiral $\mathrm{SC}_{1}$ & $x$ & $\checkmark$ & $\checkmark$ & 0 & No & $\Delta_{c h}^{1}$ \\
\hline$\Psi \sigma_{2} \tau_{0} \Psi$ & Chiral $\mathrm{SC}_{2}$ & $\checkmark$ & $\checkmark$ & $\times$ & 0 & No & $\Delta_{c h}^{2}$ \\
\hline$\Psi \sigma_{(0,1,3)} \tau_{2} \Psi$ & Triplet SC & $\times$ & $\checkmark$ & $\times$ & 1 & No & $\Delta_{t}$ \\
\hline
\end{tabular}

\section{BROKEN-SYMMETRY PHASES}

Since the DOS of the 2D ASM vanishes as $Q(E) \sim \sqrt{E}$, the noninteracting QCP separating a Dirac semimetal and a band insulator remains stable against sufficiently weak short-range interactions. Nevertheless, beyond a critical strength of the interactions, the ASM can become unstable toward the formation of various broken-symmetry phases. Here, we consider all possible phases, including both particle-hole and excitonic, as well as particle-particle or superconducting orders. However, we restrict ourselves to the momentum-independent (local or intra-unit-cell) orderings. Then, the ASM all together supports eight excitonic and four pairing orders, enumerated in Table II. We distinguish these with the labels in the order-parameter (OP) column of this table. The transformations of these fermion bilinears under the symmetry generators of the noninteracting system are also highlighted in Table II.

One can add eight particle-hole channel fermion bilinears to the noninteracting Hamiltonian. Of these, only the anisotropy parameter $\Delta_{2}^{s}$ that tunes through the Diracsemimetal to band-insulator transition preserves all discrete and continuous symmetries. The charge density $\Delta_{0}^{s}, x$ current $\Delta_{1}^{s}$, and $x$ spin current $\Delta_{1}^{t}$ each break at least one discrete or continuous symmetry, but these conserved symmetry currents do not represent true order parameters. The remaining four bilinears are order parameters for the following broken-symmetry phases: sublattice-staggered $\mathrm{CDW} \Delta_{3}^{s}$, sublattice-staggered collinear (Néel) AFM $\Delta_{3}^{t}$, uniform ferromagnet $\Delta_{0}^{t}$, and spin bond-density wave (spin BDW) $\Delta_{2}^{t}$. The last of these corresponds to spin-dependent hopping, since it preserves SLS [see Eq. (12)].

Only the CDW and AFM order parameters induce a fully gapped spectrum in the ordered phase. We, therefore, anticipate CDW and AFM to be the dominant orders for the ASM with strong repulsive interactions [see Figs. 4(b) and 12], since they optimally minimize the free energy (at least at $T=0$ ). Nevertheless, it is worth understanding the effects of other fermionic bilinears in the ordered phase. For example, a nonzero vacuum expectation value for $\Delta_{1}^{s}$ renormalizes the Fermi velocity in the $x$ direction, while a ferromagnetic compensated ASM is realized for $\Delta_{0}^{t} \neq 0$. In this phase, perfectly nested electronlike and holelike Fermi surfaces arise for opposite projections of the electron spin. Thus, such a compensated semimetallic phase can undergo a subsequent BCS-like weak coupling instability towards the formation of an AFM phase [68-71]. However, the order parameter in this AFM phase will be locked in the spin-easy plane, perpendicular to the ferromagnetic moment in the compensated semimetal. For $\Delta_{1}^{t} \neq 0$, the Fermi velocity in the $x$ direction acquires opposite corrections for the two projections of electronic spin, while a nonzero value of $\Delta_{2}^{t}$ yields both the Dirac semimetal and band insulator, but for opposite spin projections.

Sufficiently strong attractive interactions could induce various superconducting phases. When we restrict ourselves to local (momentum-independent or intra-unitcell) pairings, all together, the ASM supports only three 
spin-singlet and one spin-triplet pairing. Among them, only the singlet $s$-wave pairing $\Delta_{s}$ gives rise to a fully gapped quasiparticle spectrum. Thus, we expect the rest of the pairings to be energetically inferior to the singlet $s$-wave superconductor. We will demonstrate that, for strong attractive interactions, the ASM becomes unstable towards the formation of this $s$-wave state [see Figs. 4(b) and 12].

\section{INTERACTING THEORY: MINIMAL MODEL}

We now consider the minimal model of electronic interactions in the 2D ASM separating the Dirac semimetal and band insulator, compatible with the symmetry of the system, discussed in Sec. II B. We discuss spinless and spin-1/2 fermions separately [72].

\section{A. Spinless fermions}

For spinless fermions, generic local four-fermion interactions are described by four quartic terms,

$$
H_{\text {int }}^{\text {spinless }}=-\int d^{2} \mathbf{r} \sum_{\mu=0}^{3}\left[g_{\mu}\left(\psi^{\dagger} \tau_{\mu} \psi\right)^{2}\right] .
$$

In this notation, $g_{\mu}>0$ represents a repulsive electronelectron interaction that promotes condensation of the corresponding Hermitian fermion bilinear $\left\langle\psi^{\dagger} \tau_{\mu} \psi\right\rangle$. However, not all quartic terms are linearly independent. The Fierz identity mandates that generic interactions for spinless fermions can be captured by only one local four-fermion interaction (see Appendix F 1). Nevertheless, the final outcome regarding the fate of the critical fermions in the ASM at strong coupling depends upon the bilinear used to decouple the interactions (in mean field theory), as discussed in Sec. V.

\section{B. Spin-1/2 electrons}

Since we throughout assume spin to be a good quantum number, a collection of interacting spin- $1 / 2$ electrons in the ASM in the presence of generic short-range interactions is described in terms of eight quartic terms:

$$
H_{\mathrm{int}}^{\prime}=-\int d^{2} \mathbf{r} \sum_{\mu=0}^{3}\left[\begin{array}{c}
g_{\mu}^{s}\left(\Psi^{\dagger} \sigma_{0} \tau_{\mu} \Psi\right)^{2} \\
+g_{\mu}^{t}\left(\Psi^{\dagger} \vec{\sigma} \tau_{\mu} \Psi\right)^{2}
\end{array}\right] .
$$

Again, not all quartic terms are independent. As shown in Appendix F 2, a maximum of four independent interactions are possible for spin-1/2 fermions. With a judicious choice (see Sec. VI), we define the interacting Hamiltonian as

$$
\begin{aligned}
H_{\text {int }}=- & \int d^{2} \mathbf{r}\left[g_{1}^{s}\left(\Psi^{\dagger} \sigma_{0} \tau_{1} \Psi\right)^{2}+g_{3}^{s}\left(\Psi^{\dagger} \sigma_{0} \tau_{3} \Psi\right)^{2}\right. \\
& \left.+g_{2}^{t}\left(\Psi^{\dagger} \vec{\sigma} \tau_{2} \Psi\right)^{2}+g_{3}^{t}\left(\Psi^{\dagger} \vec{\sigma} \tau_{3} \Psi\right)^{2}\right] .
\end{aligned}
$$

In Sec. VI, we will perform a RG calculation with $H_{\text {int }}$ to understand the effect of electronic interactions in the spinful 2D ASM.
It is instructive to express the interacting theory in terms of a different set of four couplings that will allow us to connect with well-established results for spin-1/2 electrons in one dimension, which in our formalism is achieved by setting $\epsilon=0$ and taking $n \rightarrow \infty$ in the RG analysis. We can cast the interacting Hamiltonian $H_{\text {int }}$ in terms of fourfermion interactions that capture the physics of spin- $1 / 2$ electrons in one dimension most efficiently, according to

$$
H_{\text {int }}^{1 D}=\int d^{2} \mathbf{r}\left[U_{N} \mathcal{O}_{N}+U_{A} \mathcal{O}_{A}+W \frac{1}{2}\left(\mathcal{O}_{W}+\overline{\mathcal{O}}_{W}\right)+X \mathcal{O}_{X}\right],
$$

where $U_{N}, U_{A}, W$, and $X$ are coupling constants, respectively, for (i) an $\mathrm{SU}(2)_{1}$ spin current-current interaction (denoted by $\mathcal{O}_{N}$ ), (ii) a U(1) charge current-current interaction (denoted by $\mathcal{O}_{A}$ ), (iii) umklapp interactions [denoted by $\left.\left(\mathcal{O}_{W}+\overline{\mathcal{O}}_{W}\right) / 2\right]$, and (iv) $\mathrm{U}(1)$ stress tensor interactions (denoted by $\mathcal{O}_{X}$ ). The precise definition of these operators is relegated to Appendix D; see Eq. (D2). The correspondence between the four coupling constants, appearing in Eqs. (17) and (16), is

$$
\begin{aligned}
U_{N} & =g_{3}^{s}-g_{2}^{t}-g_{3}^{t}, \quad U_{A}=2 g_{1}^{s}+g_{3}^{s}+3 g_{2}^{t}+3 g_{3}^{t}, \\
W & =-g_{3}^{s}-3 g_{2}^{t}+3 g_{j 3}, \quad X=-g_{1}^{s},
\end{aligned}
$$

or

$$
\begin{aligned}
& g_{1}^{s}=-X, \quad g_{2}^{t}=\frac{1}{12}\left[-3 U_{N}+U_{A}-2 W+2 X\right], \\
& g_{3}^{s}=\frac{1}{4}\left[3 U_{N}+U_{A}+2 X\right], \quad g_{3}^{t}=\frac{1}{6}\left[U_{A}+W+2 X\right] .
\end{aligned}
$$

This mapping will prove useful to connect with the physics of interacting electrons in 1D, as discussed in Sec. VI.

\section{INTERACTING SPINLESS FERMIONS}

We first discuss the effects of electronic interactions on spinless fermions. As shown in the previous section, generic local interactions for spinless fermions are described by only one quartic term. However, we will demonstrate that, for stronger interaction, the direct transition between the Dirac semimetal and band insulator can either (i) get replaced by a first-order transition or (ii) get avoided by an intervening broken-symmetry phase, depending on how we address the effects of strong electronelectron interactions.

\section{A. Topological first-order transition}

We first illustrate the possibility of the first-order transition between the Dirac semimetal and band insulator at strong interaction, for which we consider the interacting Hamiltonian with local interaction $g_{2}$ [see Eq. (14)]. This outcome can be demonstrated from the following meanfield or large- $N$ free energy 


$$
\frac{F}{N}=\frac{\Sigma^{2}}{2 g_{2}}-\int \frac{d^{2} \mathbf{k}}{(2 \pi)^{2}} \sqrt{v^{2} k_{x}^{2}+\left(b k_{y}^{2}+\Delta+\Sigma\right)^{2}},
$$

where $N$ is the number of two-component spinors, obtained after performing a Hubbard-Stratonovich transformation of the four-fermion interaction proportional to $g_{2}$ in favor of a bosonic field $\Sigma=\left\langle\psi^{\dagger} \tau_{2} \psi\right\rangle$ and, subsequently, integrating out the critical fermions. The parameter $N$ (flavor number) should not be confused with $n$ [controlling the degree of anisotropy in ASM, Eq. (6)]. To proceed with the calculation, let us now define a set of variables according to

$$
v k_{x}=\rho \cos \theta, \quad b k_{y}^{2}=\rho \sin \theta,
$$

with $0 \leq \theta \leq \pi$ and $0<\rho<E_{\Lambda}$, where $E_{\Lambda}$ is the highenergy cutoff up to which the quasiparticle dispersion is anisotropic (given by $E_{\mathbf{k}}$ ). In terms of dimensionless variables, defined as

$$
x=\frac{\rho}{E_{\Lambda}}, \quad m=\frac{\Delta}{E_{\Lambda}}, \quad \sigma=\frac{\Sigma}{E_{\Lambda}}, \quad \lambda_{2}=\frac{2 g_{2} \sqrt{E_{\Lambda}}}{(2 \pi)^{2} v \sqrt{b}},
$$

(here $\sigma$ should not be confused with Pauli matrices) and the dimensionless free-energy density $f=F(2 \pi)^{2} 2 v \sqrt{b} /$ $\left(2 N E_{\Lambda}^{5 / 2}\right)$, we can express the above equation as

$$
\begin{aligned}
f= & \frac{\sigma^{2}}{\lambda_{2}}-\int_{0}^{1} \sqrt{x} d x \int_{0}^{1} d y \frac{1}{\left(1-y^{2}\right)^{3 / 4}} \\
& \times \sqrt{x^{2} y^{2}+\left(x \sqrt{1-y^{2}}+m+\sigma\right)^{2}} .
\end{aligned}
$$

We numerically minimize the free energy, and the resulting phase diagram is shown in Fig. 5(a). Nonetheless, the salient features of the phase diagram can be appreciated by expanding the above free energy in powers of $\sigma$, yielding

$$
f=\left[\frac{m^{2}}{\lambda_{2}}+f_{0}\right]-\sigma\left[\frac{m}{\lambda_{2}}-f_{1}\right]+\sum_{j \in \text { integer }} \sigma^{j} f_{j},
$$

after shifting the variable $\sigma+m \rightarrow \sigma$. It should, however, be noted that such expansion of the free-energy density in powers of $\sigma$ is not a well-defined procedure and, for $j>4$, the $f_{j}$ 's are nonanalytic functions. But, most importantly, the free energy $f$ contains all odd powers of $\sigma$. When the interaction is sufficiently weak, the $\sigma$ field does not condense, and the profile of the free energy possesses a single global minimum, as shown in Fig. 5(b). Consequently, the nature of the direct, continuous transition between the Dirac semimetal and band insulator remains unchanged at weak coupling. In this regime, only the term linear in $\sigma$ is important, and $\delta=\lambda_{2} f_{1}$ defines the phase

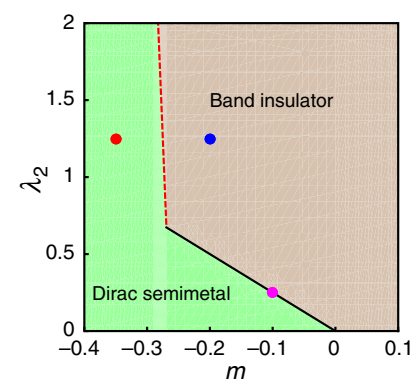

(a)

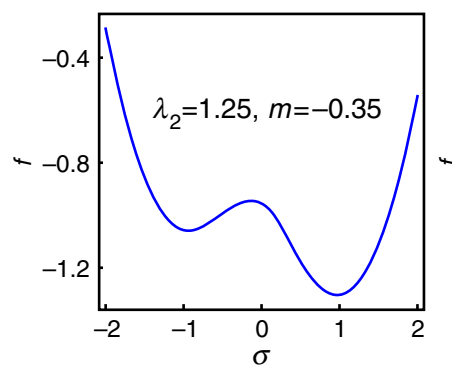

(c)

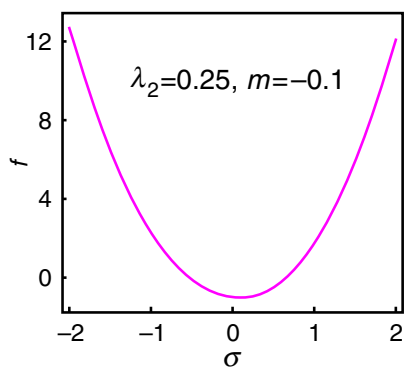

(b)

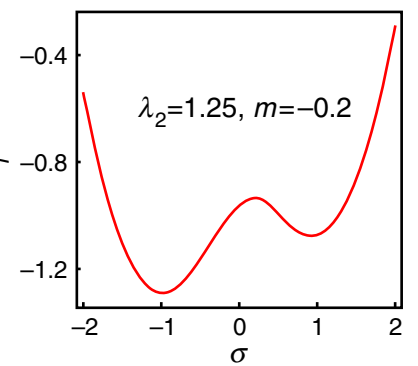

(d)
FIG. 5. (a) Phase diagram of interacting spinless fermions obtained by minimizing the free energy, shown in Eq. (22). The profile of the (dimensionless) free energy at three points in the phase diagram, marked in Fig. 5(a), is shown in panels (b)-(d). Values of various parameters are displayed in the corresponding figures. Here, $\lambda_{2}$ and $m$ are the dimensionless interaction coupling and anisotropy parameter, and $\sigma$ is the interaction-induced condensate of the anisotropy parameter (also dimensionless) [see Eq. (21)]. (b) For weak enough interaction, the free energy possesses only one global minimum and, thus, the direct transition between Dirac semimetal and band insulator is continuous. The universality class of this transition is determined by the two-dimensional anisotropic semimetal. For stronger interaction, the free-energy profile develops two inequivalent local minima, and, respectively, in panels (c) and (d), we display the free energy on the insulating and Dirac semimetal sides of the transition. Consequently, the direct transition between these two phases at strong interaction becomes first order in nature [represented by the red dashed line in (a)].

boundary between these two phases; this is represented by the solid black line in Fig. 5(a).

On the other hand, beyond a critical strength of the interaction $\lambda_{2}>\lambda_{2}^{*} \sim 1$, the $\sigma$ field acquires a finite vacuum expectation value and all powers (including the odd ones) of $\sigma$ in $f$ become important. In this regime, the profile of the free energy accommodates two inequivalent minima, as shown in Figs. 5(c) and 5(d), respectively, on the band-insulator and Dirac-semimetal sides of the phase diagram. As a result, the direct transition between these two phases at strong coupling becomes a fluctuation-driven first-order transition along the dashed red line in Fig. 5(a). [73] When the Dirac semimetal is separated from the band insulator by a first-order transition, there exists no ASM at the transition. A similar first-order transition at strong coupling has also been predicted in twoand three-dimensional topological insulators [75-78] and 
also in a three-dimensional Weyl material residing in proximity to the semimetal to band-insulator QCP [65].

It is worth mentioning that a first-order transition has recently been observed in the pressured organic compound $\alpha$-(BEDT-TTF $)_{2} \mathrm{I}_{3}$ [26]. Thus, our theory provides a possible explanation of this experimental observation.

\section{B. Continuous quantum phase transition}

Next, we will demonstrate the second possibility regarding the fate of the direct transition between the Dirac semimetal and band insulator, when strong interactions cause nucleation of a broken-symmetry phase that masks the direct transition between these two symmetry-preserving phases [see Fig. 2(b)]. For spinless fermions, only the CDW phase gives rise to a fully gapped spectrum; inside this ordered phase, $\left\langle\psi^{\dagger} \tau_{3} \psi\right\rangle \neq 0$. Thus, CDW order is energetically advantageous at temperature $T=0$ for sufficiently large $g_{3}>0$ [Eq. (14)]. Next, we will establish the onset of such a CDW phase by carrying out a RG calculation on the interacting model with local interaction $g_{3}$. The imaginarytime action for such an interacting system reads

$$
\mathcal{S}=\mathcal{S}_{0}-g_{3} \int d t d^{2} \mathbf{r}\left(\psi^{\dagger} \tau_{3} \psi\right)^{2}
$$

After accounting for perturbative corrections to the quadratic order in $g_{3}$ (computing the one-loop diagrams shown in Fig. 6) and integrating out the fast Fourier modes within the Wilsonian shell $E_{\Lambda} e^{-l}<\sqrt{\omega^{2}+v^{2} k_{x}^{2}}<E_{\Lambda}$ and $0<\left|k_{y}\right|<\infty$, we arrive at the following RG flow equations,

$$
\begin{aligned}
& \frac{d \hat{\Delta}}{d l}=\hat{\Delta}+\lambda_{3}\left[\hat{\Delta}\left[2 f_{1}(n)+f_{2}(n)\right]+f_{3}(n)\right], \\
& \frac{d \lambda_{3}}{d l}=-\epsilon \lambda_{3}+4 \lambda_{3}^{2} f_{2}(n),
\end{aligned}
$$

where $\hat{\Delta}=\Delta / E_{\Lambda}$ and $\lambda_{3}=g_{3} E_{\Lambda}^{\epsilon} /\left(8 \pi^{2} v b^{\epsilon}\right)$ are dimensionless parameters. Here, $E_{\Lambda}$ is the energy cutoff for $\omega$ and $v\left|k_{x}\right|$, and $\omega$ is the Matsubara frequency. The functions appearing in the RG flow equations are (see Appendix E for details)

$f_{1}(n)=\frac{\pi(2 n-1) \csc \left(\frac{\pi}{2 n}\right)}{4 n^{2}}=1-\frac{1}{2 n}+\mathcal{O}\left(n^{-2}\right)$,

$f_{2}(n)=\frac{\pi \csc \left(\frac{\pi}{2 n}\right)}{2 n^{2}}=\frac{1}{n}+\mathcal{O}\left(n^{-2}\right)$,

$f_{3}(n)=\frac{\pi \sec \left(\frac{\pi}{2 n}\right)}{n}=\frac{\pi}{n}+\mathcal{O}\left(n^{-2}\right)$.

Therefore, as $n \rightarrow \infty$, only the contribution from $f_{1}(n)$ survives, while $f_{2}(n)$ and $f_{3}(n)$ capture subleading logarithmic divergences. The above coupled flow equations support only two fixed points:

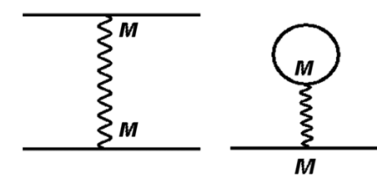

(a)

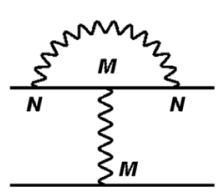

(e) (b)

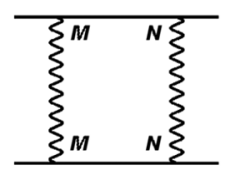

(f)

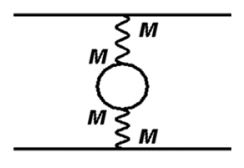

(d)

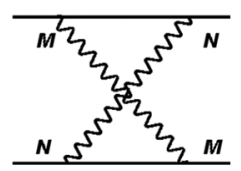

(g)
FIG. 6. (a) Bare four-fermion interaction vertex $\left(\psi^{\dagger} M \psi\right)^{2}$ (for spinless fermions) or $\left(\Psi^{\dagger} M \Psi\right)^{2}$ (for spin-1/2 electrons). The Feynman diagrams contributing to the renormalization group flow equations to the leading order in $\epsilon$ and $1 / n$ expansion are shown as (b) $-(\mathrm{g})$. Here, $M$ and $N$ are $2 \times 2$ (for spinless fermions) and $4 \times 4$ (for spin- $1 / 2$ electrons) Hermitian matrices.

(1) $\left(\hat{\Delta}, \lambda_{3}\right)=(0,0)$ corresponds to the noninteracting system, the ASM critical point that connects the Dirac semimetal to the symmetry-preserving band insulator.

(2) $\left(\hat{\Delta}, \lambda_{3}\right)=(-(\pi / 4),(n / 4)) \epsilon$, which, on the other hand, stands as an interacting multicritical point in the $\left(\hat{\Delta}, \lambda_{3}\right)$ plane, where the Dirac-semimetal, band-insulator, ASM, and CDW phases meet. Therefore, this point controls a continuous quantum phase transition of critical excitations residing at the phase boundary between the Dirac semimetal and band insulator towards the formation of the CDW phase. The RG flow and the phase diagram are, respectively, shown in Figs. 7(a) and 7(b).

We now show how to compute the location of the interacting multicritical point from Eq. (25). Respectively, $d \hat{\Delta} / d l=0$ and $d \lambda_{3} / d l=0$ lead to

$$
\begin{aligned}
\Delta_{*} & =-\frac{\lambda_{3, *}}{1+\lambda_{3, *}\left[2 f_{1}(n)+f_{2}(n)\right]} f_{3}(n) \\
& =-f_{3}(n) \lambda_{3, *} \quad\left(1-\lambda_{3, *}\left[2 f_{1}(n)+f_{2}(n)\right]+\cdots\right), \\
\lambda_{3, *} & =\frac{\epsilon}{4 f_{2}(n)}=\frac{n}{4} \epsilon,
\end{aligned}
$$

where quantities with the subscript $*$ denote their fixed point values. Since $\lambda_{3, *} \sim \epsilon$ for a systematic extraction of $\hat{\Delta}_{*}$ to the leading order in $\epsilon$ (interaction mediated shift of the band parameter), we only need to account for the first term in the above expression for $\hat{\Delta}_{*}$, yielding [79]

$$
\hat{\Delta}_{*}=-f_{3}(n) \lambda_{3, *}+\mathcal{O}\left(\lambda_{3, *}^{2}\right)=-\frac{\pi}{4} \epsilon+\mathcal{O}\left(\epsilon^{2}\right),
$$

leading to the result announced above. The remaining terms in the expression of $\hat{\Delta}_{*}$ from Eq. (27) yield contributions at 


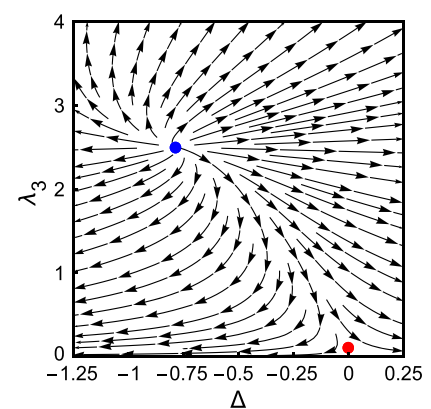

(a)

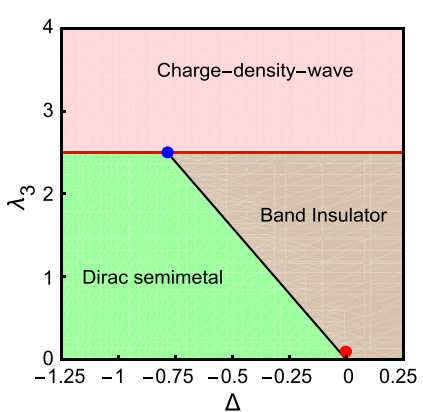

(b)
FIG. 7. (a) The renormalization group flow diagram obtained from Eq. (25) for spinless fermions. (b) The corresponding phase diagram. The red (blue) dot corresponds to the noninteracting critical (interacting multicritical) point. The (dimensionless) coupling constant $\lambda_{3}$ and band parameter $\hat{\Delta}$ in panels (a) and (b) are measured in units of $\epsilon$ and, in the RG flow equations [see Eq. (25)], we substitute $n=10$ in $f_{j}(n)$.

least $\mathcal{O}\left(\epsilon^{2}\right)$. Therefore, while determining the location of the multicritical point, $\mathrm{RG}$ flow, and phase diagram in the $\left(\hat{\Delta}, \lambda_{3}\right)$ plane, we only accounted for the terms proportional to $\hat{\Delta}$ and $\lambda_{3}$ and neglected the contribution proportional to $\hat{\Delta} \lambda_{3}$ in $d \hat{\Delta} / d l$. We follow the same procedure for spin- $1 / 2$ electrons discussed in the next section.

From the leading order in $\epsilon$ and $1 / n$ expansions, we can only estimate the correlation length exponent $(\nu)$ for the ASM-CDW continuous transition, yielding

$$
\nu^{-1}=\left.\frac{d}{d \lambda_{3}}\left[\frac{d \lambda_{3}}{d l}\right]\right|_{\lambda_{3}=\lambda_{3}^{*}}=\epsilon,
$$

where $\lambda_{3}^{*}=(n / 4) \epsilon$ represents the strength of four-fermion interactions at the multicritical point. Hence, for the physically relevant situation with $\epsilon=1 / 2$ and $n=2$, we obtain $\nu=2$. On the other hand, when the Dirac semimetal undergoes a continuous transition into the CDW phase, $\nu=1$ (to one-loop order) [66]. Thus, the universality class of the critical excitation-CDW transition is fundamentally a new one.

However, we note that the multicritical point describing the transition is not strictly controlled by a small parameter as $\lambda_{3}^{*} \sim n \epsilon \sim 1$. This outcome is specific (and to certain extent pathological) for spinless two-component fermions. For spin-1/2 electrons, we show in the next section that all of the interacting QCPs and the associated critical properties are simultaneously controlled by two small parameters $\epsilon$ and $1 / n$.

The spinless case gives an order-one coupling strength at the multicritical point because the correction in Eq. (25b) vanishes in the $n \rightarrow \infty$ limit. This is true to all orders in $\lambda_{3}$, since the interaction in Eq. (24) is equivalent to a $\mathrm{U}(1)$ current-current (Thirring [34]) perturbation. This is an exactly marginal Luttinger interaction in the $1 \mathrm{D}$ limit [42,43], which obtains for $\epsilon=0$ and $n \rightarrow \infty$. Thus, the
ASM-CDW quantum phase transition is solely captured by the subleading divergence arising from $f_{2}(n)$, which can be directly tested, at least in quantum Monte Carlo simulation, with only NN interaction in a uniaxially stressed honeycomb lattice $[63,64]$ [with $t_{2}=2 t_{1}$; see Fig. 1(b)]. By contrast, for isotropic two-component massless Dirac fermions, the RG flow equation vanishes to at least order $\lambda_{3}^{3}$ [80], although it is believed that there exists a possibly continuous semimetalinsulator transition at finite interaction strength $[81,82]$. Thus far, this issue remains unsettled. On the other hand, our leading-order RG calculation predicts a continuous anisotropic semimetal-CDW transition.

\section{First- vs. second-order transitions}

We have identified two incompatible scenarios for the strong coupling physics in the spinless case, wherein the ASM is replaced by either a first-order transition or the CDW phase. These conclusions are reached via calculations with different control parameters: the number of flavors $N$ for the first-order transition and the curvature of the dispersion along $k_{y}$ (characterized by the $n$ ) for the intervening CDW phase. Since we must set $N=1$ and $n=2$ for the spinless model, both results obtain at strong coupling, and we cannot make a rigorous mathematical argument that one scenario is more likely. However, microscopic considerations provide some physical intuition that can favor one or the other.

For a spinless ASM obtained from a microscopic strained honeycomb lattice model that is free of frustration (e.g., a $t-V$ model with nearest-neighbor hopping and densitydensity interactions only), the CDW order is expected to preempt the topological first-order transition between the Dirac semimetal and band insulator, since the CDW order completely gaps out critical excitations, producing a uniform mass gap in the spectrum. This can be tested in a quantum Monte Carlo simulation [63,64]. However, we cannot completely rule out the first-order transition, since bandwidth renormalization and/or the suppression of the quasiparticle weight may become crucial to determine the ultimate fate of the strongly interacting ASM. These effects are not included in the one-loop RG.

For spin-1/2 electrons, generic local interactions live in a four-dimensional coupling constant space (see the next section). We can again gain some intuition by considering a particular microscopic model, e.g., the strained honeycomb lattice extended Hubbard model [see Fig. 4(b)]. Again, this model is "frustration free," and the fully gapped mass orders CDW, AFM, and $s$-wave pairing are expected to occur at strong coupling. Nucleation of these mass orders via a continuous phase transition is energetically superior over the first-order transition out of the Dirac semimetal, since the ordered phases fully gap the spectrum (maximal gain of condensation energy).

We, therefore, conclude that if the extended Hubbard model can be realized in strained graphene or an anisotropic 
honeycomb optical lattice for ultracold fermions, the firstorder transition will be unlikely. For a material with different microscopics [e.g., the organic compound $\alpha$-(BEDT$\mathrm{TTF})_{2} \mathrm{I}_{3}$, the Dirac-semimetal to band-insulator transition could occur at strong coupling in a corner of the fourdimensional interaction parameter space, where the firstorder transition is actually preferred. It is a nontrivial task to identify a microscopic model that can exhibit the proposed first-order transition in this high-dimensional coupling space. Finally, it must be emphasized that the first-order transition we discuss here between two symmetry-preserving phases (Dirac semimetal and band insulator) is fundamentally different from the one between two distinct broken-symmetry phases. Therefore, such a fluctuation-driven first-order transition cannot be captured by a RG calculation, and the computation of free-energy density (nonperturbative analysis in the large- $N$ limit) is necessary [74].

\section{SPIN-1/2 ELECTRONS}

Next we perform the RG calculation to the leading order in $\epsilon$ and $1 / n$ starting from the interacting $H_{\text {int }}$ [defined in Eq. (16)] for spin-1/2 electrons. We made a judicious choice in selecting the linearly independent coupling constants in $H_{\text {int }}$. Note that we did not choose $g_{2}^{s}$ as one of the four independent couplings, since as we know, when this coupling constant is strong enough, the Dirac-semimetal to bandinsulator quantum phase transition becomes a fluctuationdriven first-order transition (following the analysis presented in Sec. VA but now for spinful electrons, which can be accomplished by taking $N \rightarrow 2 N$ ). Nonetheless, as we will discuss in the next section, the source term $\Delta_{2}^{s}$ (corresponding to the anisotropy parameter) never displays the leading divergence. Thus, we can proceed with the following RG analysis tailored to address continuous phase transitions into
TABLE III. Location of the fixed points obtained from the RG flow equations as we set $n \rightarrow \infty$ in Eqs. (30) and (31), in the perturbative quadratic-order corrections to the four-fermion coupling constants. The resulting RG flows are displayed in Eq. (32). The noninteracting semimetal is labeled $\mathrm{FP}_{1}$ and has only one unstable direction; it controls the direct quantum phase transition between the Dirac semimetal and band insulator for sufficiently weak interactions. By contrast, each of $\mathrm{FP}_{2,3,4}$ has two unstable directions and $\mathrm{FP}_{5,6}$ has three unstable directions, and these correspond to interacting multicritical points. The penultimate rightmost column shows the value of the band parameter $\hat{\Delta}$ at each such fixed point. If we follow the RG trajectory along which the renormalized band parameter is kept fixed, i.e., $\hat{\Delta}=0$, then $\mathrm{FP}_{2,3,4}$ represent interacting quantum critical points with only one unstable direction and $\mathrm{FP}_{5,6}$ become bicritical points with two unstable directions. A schematic plot of these fixed points is shown in Fig. 9. The last column shows the number of unstable directions (UDs) at a given fixed point in the $\hat{\Delta}=0$ hyperplane, as well as in the fivedimensional coupling constant space (shown in parentheses).

\begin{tabular}{lcccc}
\hline \hline Fixed point & $\left(\lambda_{1}^{s}, \lambda_{3}^{s}, \lambda_{2}^{t}, \lambda_{3}^{t}\right)$ & $\left(U_{N}, U_{A}, W, X\right)$ & $\hat{\Delta}$ & $\mathrm{UDs}$ \\
\hline $\mathrm{FP}_{1}$ & $(0,0,0,0)$ & $(0,0,0,0)$ & 0 & $0(1)$ \\
$\mathrm{FP}_{2}$ & $\left(0, \frac{3}{4},-\frac{1}{4}, 0\right) \epsilon$ & $(1,0,0,0) \epsilon$ & 0 & $1(2)$ \\
$\mathrm{FP}_{3}$ & $\left(0, \frac{1}{4}, \frac{1}{4}, 0\right) \epsilon$ & $(0,1,-1,0) \epsilon$ & 0 & $1(2)$ \\
$\mathrm{FP}_{4}$ & $\left(0, \frac{1}{4},-\frac{1}{12}, \frac{1}{3}\right) \epsilon$ & $(0,1,1,0) \epsilon$ & 0 & $1(2)$ \\
$\mathrm{FP}_{5}$ & $(0,1,0,0)$ & $(1,1,-1,0) \epsilon$ & 0 & $2(3)$ \\
$\mathrm{FP}_{6}$ & $\left(0,1,-\frac{1}{3}, \frac{1}{3}\right) \epsilon$ & $(1,1,1,0) \epsilon$ & 0 & $2(3)$ \\
\hline \hline
\end{tabular}

broken-symmetry phases, leaving aside the possibility of a first-order transition as an alternative scenario. Following the same procedure described for spinless fermions in Sec. V B (see Fig. 6 for the relevant Feynman diagrams), we arrive at the following RG equations:

$$
\begin{aligned}
& \frac{d \hat{\Delta}}{d l}=\hat{\Delta}+\left(\lambda_{1}^{s}+\lambda_{3}^{s}-3 \lambda_{2}^{t}+3 \lambda_{3}^{t}\right) \times\left(\left[2 f_{1}(n)+f_{2}(n)\right] \hat{\Delta}+f_{3}(n)\right), \\
& \frac{d \lambda_{1}^{s}}{d l}=-\epsilon \lambda_{1}^{s}+\frac{\lambda_{1}^{s}}{2}\left[\lambda_{1}^{s}+\lambda_{3}^{s}+3 \lambda_{2}^{t}+3 \lambda_{3}^{t}\right] f_{2}(n)-3 \lambda_{1}^{s} \lambda_{2}^{t} f_{2}(n) \\
& \frac{d \lambda_{3}^{s}}{d l}=-\epsilon \lambda_{3}^{s}+\frac{\lambda_{3}^{s}}{2}\left[\lambda_{1}^{s}+\lambda_{3}^{s}+3 \lambda_{2}^{t}-3 \lambda_{3}^{s}\right] \times\left[2 f_{1}(n)+f_{2}(n)\right]+f_{1}(n)\left[-\lambda_{1}^{s} \lambda_{3}^{s}-3 \lambda_{3}^{s} \lambda_{2}^{t}+3\left(\lambda_{2}^{t}\right)^{2}\right. \\
& \left.-3 \lambda_{2}^{t} \lambda_{3}^{t}+3\left(\lambda_{3}^{t}\right)^{2}\right]+f_{2}(n)\left[3 \lambda_{1}^{s} \lambda_{2}^{t}+3 \lambda_{2}^{t} \lambda_{3}^{t}+\frac{1}{2}\left(\left[\lambda_{1}^{s}\right]^{2}+\left[\lambda_{3}^{s}\right]^{2}+3\left[\lambda_{2}^{t}\right]^{2}+3\left[\lambda_{3}^{t}\right]^{2}\right)\right], \\
& \frac{d \lambda_{2}^{t}}{d l}=-\epsilon \lambda_{2}^{t}+\frac{\lambda_{2}^{t}}{2}\left[\lambda_{1}^{s}+\lambda_{3}^{s}+3 \lambda_{2}^{t}-\lambda_{3}^{t}\right] \times\left[2 f_{1}(n)-f_{2}(n)\right]+f_{1}(n)\left[\frac{1}{3} \lambda_{1}^{s} \lambda_{3}^{s}-\lambda_{1}^{s} \lambda_{3}^{t}+\lambda_{3}^{s} \lambda_{2}^{t}-\left[\lambda_{2}^{t}\right]^{2}-\lambda_{2}^{t} \lambda_{3}^{t}-\left[\lambda_{2}^{t}\right]^{2}\right] \\
& +f_{2}(n)\left[\lambda_{3}^{s} \lambda_{3}^{t}-\frac{1}{6}\left(\left[\lambda_{1}^{s}\right]^{2}+\left[\lambda_{3}^{s}\right]^{2}+3\left[\lambda_{2}^{t}\right]^{2}+3\left[\lambda_{3}^{t}\right]^{2}\right)\right] \\
& \frac{d \lambda_{3}^{t}}{d l}=-\epsilon \lambda_{3}^{t}+\frac{\lambda_{3}^{t}}{2}\left[\lambda_{1}^{s}-\lambda_{3}^{s}-\lambda_{2}^{t}+\lambda_{3}^{t}\right] \times\left[2 f_{1}(n)+f_{2}(n)\right]+f_{1}(n)\left[-\frac{1}{3} \lambda_{1}^{s} \lambda_{3}^{s}-\lambda_{1}^{s} \lambda_{2}^{t}-2 \lambda_{2}^{t} \lambda_{3}^{t}\right] \\
& +f_{2}(n)\left[\lambda_{1}^{s} \lambda_{2}^{t}+\lambda_{3}^{s} \lambda_{2}^{t}-2 \lambda_{2}^{t} \lambda_{3}^{t}+\frac{1}{6}\left(\left[\lambda_{1}^{s}\right]^{2}+\left[\lambda_{3}^{s}\right]^{2}+3\left[\lambda_{2}^{t}\right]^{2}+3\left[\lambda_{3}^{t}\right]^{2}\right)\right]
\end{aligned}
$$




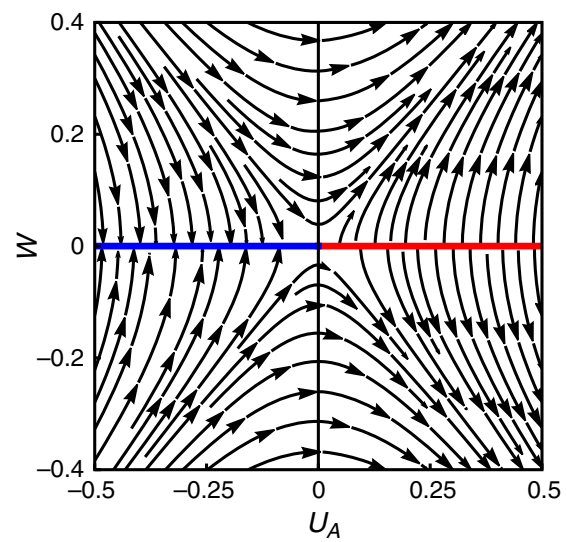

FIG. 8. RG flow diagram in the $\left(U_{A}, W\right)$ plane in the strict one-dimensional limit [obtained by taking $n \rightarrow \infty$ in Eq. (31) and setting $\epsilon=0$ in Eq. (32)]. The stable (unstable) line of fixed points is indicated in blue (red). The flow determines the charge sector Kosterlitz-Thouless transition [42].

where $\lambda_{\mu}^{a}=g_{\mu}^{a} E_{\Lambda}^{\epsilon} /\left(\pi^{2} v b^{\epsilon}\right)$ is the dimensionless coupling constant and $\hat{\Delta}=\Delta / E_{\Lambda}$ is the dimensionless band parameter, and recall that, here, $n$ can only take even integer values. The above set of RG flow equations can also be expressed in terms of the coupling constants introduced in Eq. (17) (namely, $U_{N}, U_{A}, W$, and $X$ ) according to

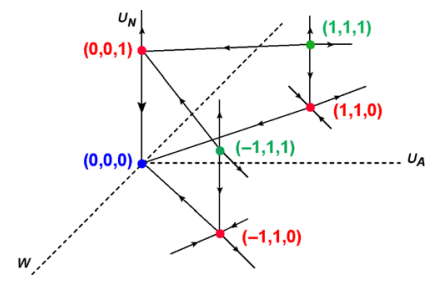

(a)

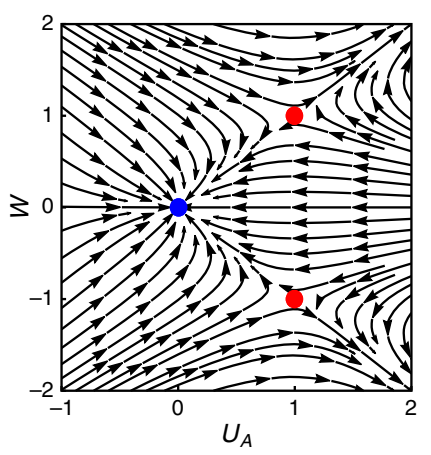

(b)
FIG. 9. (a) A schematic representation of the renormalization group flow in three-dimensional coupling constant space $\left(W, U_{A}\right.$, $\left.U_{N}\right)$ for renormalized band parameter $\hat{\Delta}(l)=0$. The stable noninteracting anisotropic semimetal fixed point at $(0,0,0)$ is shown in blue; the quantum critical points at $(0,0,1)$ and $( \pm 1,1,0)$ are shown in red; and the remaining two bicritical points at $( \pm 1,1,1)$ are shown in green. The coupling constants are measured in units of $\epsilon$ (see also Table III). (b) Deformation of the flow diagram from Fig. 8 in the $\left(U_{A}, W\right)$ plane, upon incorporating the negative engineering dimension $(-\epsilon)$ for the interaction couplings [see Eq. (32)]. Recall that all the fixed points have one additional unstable direction along $\hat{\Delta}$, which controls the transition between a Dirac semimetal and band insulator.

$$
\begin{aligned}
\frac{d \hat{\Delta}}{d l}= & \hat{\Delta}+\left[\frac{1}{2}\left(U_{A}+3 U_{N}\right)+W\right]\left(\left[2 f_{1}(n)+f_{2}(n)\right] \hat{\Delta}+f_{3}(n)\right), \\
\frac{d X}{d l}= & -\epsilon X+\frac{1}{4} X\left[U_{A}+3 U_{N}+2 W\right] f_{2}(n), \\
\frac{d U_{N}}{d l}= & -\epsilon U_{N}+U_{N}^{2} f_{1}(n)+\frac{1}{72}\left[8 U_{A}^{2}+27 U_{N}^{2}-33 U_{N} W-4 W^{2}-15 U_{N} U_{A}\right. \\
& \left.-23 U_{A} W+14 X U_{A}-12 U_{N} X-10 W X+32 X^{2}\right] f_{2}(n), \\
\frac{d U_{A}}{d l}= & -\epsilon U_{A}+W^{2} f_{1}(n)+\frac{1}{24}\left[4 U_{A}^{2}+15 U_{A} U_{N}+9 U_{N}^{2}+11 U_{A} W-3 U_{N} W+4 W^{2}-14 U_{A} X+12 U_{N} X+10 X W-8 X^{2}\right] f_{2}(n), \\
\frac{d W}{d l}= & -\epsilon W+U_{A} W f_{1}(n)+\frac{1}{24}\left[-2 U_{A}^{2}-3 U_{N} U_{A}+9 U_{N}^{2}+5 U_{A} W+15 U_{N} W\right. \\
& \left.+16 W^{2}-14 X U_{A}+12 X U_{N}+10 W X-8 X^{2}\right] f_{2}(n) .
\end{aligned}
$$

In the last set of equations, all coupling constants are dimensionless. Next, we systematically analyze the above set of RG flow equations. We addressed the effect of RG flow of $\hat{\Delta}$ in the phase diagram of the interacting ASM for the spinless case. Unless otherwise mentioned, we will work in the hyperplane defined by $\hat{\Delta}(l)=0$, where $\hat{\Delta}(l)$ is the renormalized band parameter. Therefore, all interacting fixed points with $\lambda_{j}^{s, t} \neq 0$ (see Tables III and IV and Fig. 9) have one additional unstable direction, which is $\hat{\Delta}$, and all of them are truly multicritical in nature. The functions $f_{1}(n), f_{2}(n)$, and $f_{3}(n)$ have already been defined in Eq. (26).

\section{A. Emergent one-dimensional system: Spin-charge separation}

In the limit $n \rightarrow \infty$, Eq. (31) reduces to

$$
\begin{aligned}
\frac{d U_{N}}{d l}=-\epsilon U_{N}+U_{N}^{2}, & \frac{d U_{A}}{d l}=-\epsilon U_{A}+W^{2}, \\
\frac{d W}{d l}=-\epsilon W+U_{A} W, & \frac{d X}{d l}=-\epsilon X .
\end{aligned}
$$

If we set the engineering dimension of all the four-fermion couplings $\epsilon=0$, then we recover the well-known RG flow equations for these coupling strengths in one dimension. 
TABLE IV. Location of the six fixed points tabulated in Table III after accounting for $1 / n$ corrections in Eqs. (30) and (31). The results obtain by utilizing the large- $n$ expansions for $f_{1}(n), f_{2}(n)$, and $f_{3}(n)$ [Eq. (26)]. The inclusion of $1 / n$ corrections does not change the number of fixed points (at least for large enough $n \geq 4$ ), but it eliminates the spin-charge separation. Thus, the $\epsilon$ expansion, augmented by $1 / n$ expansion, allows a controlled route to access some strong coupling phenomena (such as the quantum phase transition between the anisotropic semimetal and a broken-symmetry phase) in two spatial dimensions. The coefficients of $1 / n$ appearing in the locations of the fixed points are extracted numerically. The last column shows the number of unstable directions (UDs) at a given fixed point in the $\hat{\Delta}=0$ hyperplane as well as in the five-dimensional coupling constant space (shown in parentheses).

\begin{tabular}{lcccc}
\hline \hline $\begin{array}{l}\text { Fixed } \\
\text { point }\end{array}$ & $\left(\lambda_{1}^{s}, \lambda_{3}^{s}, \lambda_{2}^{t}, \lambda_{3}^{t}\right)$ & $\left(U_{N}, U_{A}, W, X\right)$ & $\hat{\Delta}$ & UDs \\
\hline $\mathrm{FP}_{1}$ & $(0,0,0,0)$ & $(0,0,0,0)$ & 0 & $0(1)$ \\
$\mathrm{FP}_{2}$ & $\left(0, \frac{3}{4}+(0.25 / n),-\frac{1}{4}-(0.08334 / n),(0.15 / n)\right) \epsilon$ & $(1+(0.18 / n),(0.46 / n),(0.44 / n), 0) \epsilon$ & 0 & $1(2)$ \\
$\mathrm{FP}_{3}$ & $\left(0, \frac{1}{4}+(0.63 / n), \frac{1}{4}+(0.075 / n),(0.025 / n)\right) \epsilon$ & $((0.53 / n), 1+(0.93 / n),-1-(0.78 / n), 0) \epsilon$ & {$[\pi /(2 n)] \epsilon$} & $1(2)$ \\
$\mathrm{FP}_{4}$ & $\left(0, \frac{1}{4}-(0.225 / n),-\frac{1}{12}+(0.0775 / n), \frac{1}{3}-(0.085 / n)\right) \epsilon$ & $(-(0.2175 / n), 1-(0.2475 / n), 1-(0.2625 / n), 0) \epsilon$ & $-(\pi / n) \epsilon$ & $1(2)$ \\
$\mathrm{FP}_{5}$ & $(0,1-(0.29 / n),(0.19 / n),(0.075 / n)) \epsilon$ & $(1-(0.555 / n), 1+(0.505 / n),-1-(0.055 / n), 0) \epsilon$ & $-(\pi / n) \epsilon$ & $2(3)$ \\
$\mathrm{FP}_{6}$ & $\left(0,1+(0.35 / n),-\frac{1}{3}-(0.13 / n), \frac{1}{3}-(0.5 / n)\right) \epsilon$ & $(1+(0.98 / n), 1-(1.54 / n), 1-(1.46 / n), 0) \epsilon$ & $-[(3 \pi) / n] \epsilon$ & $2(3)$ \\
\hline \hline
\end{tabular}

In this limit, the anisotropic semimetal with local interactions reduces to a decoupled collection of spin- $1 / 2$ one-dimensional "wires." Such systems exhibit spin-charge separation [42,43], and this is reflected in Eq. (32). The only spin sector interaction $U_{N}$ couples to the $\mathrm{SU}(2)_{1}$ current-current perturbation [see Eq. (D2) and the surrounding text for a precise definition]. For $\epsilon=0, U_{N}$ resides on the inflowing (outflowing) part of the spin sector Kosterlitz-Thouless separatrix for $U_{N}<0 \quad\left(U_{N}>0\right)$ $[42,43]$; spin $\mathrm{SU}(2)$ symmetry is preserved everywhere along the separatrix.

The remaining parameters couple to interaction operators that perturb the charge sector, as can be seen from the bosonization of the latter [Eq. (D3)]. $U_{A}$ and $X$ couple to the $U(1)$ current-current and $U(1)$ stress tensor operators, respectively; these modify only the Luttinger parameter and charge velocity of the free boson description, as shown in Eq. (D4). By contrast, the umklapp interaction $W$ couples to a sine-Gordon perturbation. The flow equations for $U_{A}$ and $W$ with $\epsilon=0$ are the charge sector Kosterlitz-Thouless equations. The RG flow in the $\left(U_{A}, W\right)$ plane is shown in Fig. 8.

Since spin and charge are independent, Eq. (32) with $\epsilon=0$ implies that either sector can be a gapless Luttinger liquid or gapped Mott insulator. The spin sector becomes massive when $U_{N} \rightarrow+\infty$, while the charge sector becomes massive when $U_{A} \rightarrow+\infty$ and $W \rightarrow \pm \infty$. The product of these gives four different composite phases that must be interpreted through a microscopic model. The onedimensional extended Hubbard model described by the phase diagram in Fig. 4(a) is an example; it is further reviewed in Sec. VIII, below.

The gapless charge sector Luttinger liquid phase is a stable line of fixed points, shown in Fig. 8. The tuning parameter is the interaction $U_{A}$ or, equivalently, the charge sector Luttinger parameter $K_{c}$ [Eq. (D4)]. Up to logarithmic corrections [42] that we ignore, the spin Luttinger liquid is a fixed point with $U_{N}=0$ and spin sector Luttinger parameter $K_{s}=1$.

\section{B. From 1D to $1+\epsilon$}

Moving beyond the strict 1D limit, it is instructive to reinstate the dispersion in the $y$ direction in two steps. First, we consider the influence of the nonzero scaling dimension $(-\epsilon)$ for all local four-fermion interactions in Eq. (32). With $\epsilon>0$, these RG flow equations give birth to six fixed points, tabulated in Table III and schematically shown in Fig. 9(a). All of them are located in a hyperplane defined by $X=0$ or $\lambda_{1}^{s}=0$. Note that inclusion of the nonzero scaling dimension does not destroy spin-charge separation.

The gapless charge sector Luttinger liquid phase (stable fixed line in Fig. 8) gets replaced by a stable noninteracting fixed point and two QCPs, as shown in Fig. 9(b). An additional quantum critical point appears in the spin sector at $U_{N}=\epsilon$.

Now we characterize all six fixed points in the hyperplane $\hat{\Delta}(l)=0$, cataloged in Table III. The fixed point $\mathrm{FP}_{1}$ represents the noninteracting ASM, stable against sufficiently weak generic short-range interactions. Each of $\mathrm{FP}_{2}, \mathrm{FP}_{3}$, and $\mathrm{FP}_{4}$ is characterized by only one unstable direction (in addition to $\hat{\Delta}$ ). These three fixed points correspond to interacting QCPs and are analogous to the interacting multicritical point shown in Fig. 7. They describe continuous transitions from the ASM to brokensymmetry phases.

To determine the actual nature of the broken symmetry across the various QCPs in the ASM requires inclusion of 
$1 / n$ corrections to the RG flow equations (30) or (31). These corrections are due to quantum fluctuations beyond one dimension and eliminate certain special symmetries specific to 1D, such as spin-charge separation. In Sec. VII, we will combine the RG with a scaling analysis of fermion bilinear susceptibilities in order to pin the pattern of symmetry breaking at strong coupling in an unbiased fashion.

The remaining two fixed points, $\mathrm{FP}_{5}$ and $\mathrm{FP}_{6}$, each possess two unstable directions, as shown in Fig. 9(a). In the $\hat{\Delta}(l)=0$ hyperplane, they represent interacting bicritical points, separating the basins of attraction for the various interacting QCPs. As shown in Fig. 9(a), $\mathrm{FP}_{5}$ separates the domain of attraction for $\mathrm{FP}_{2}$ and $\mathrm{FP}_{3}$, while $\mathrm{FP}_{6}$ separates $\mathrm{FP}_{4}$ and $\mathrm{FP}_{2}$. The basins of attraction for $\mathrm{FP}_{3}$ and $\mathrm{FP}_{4}$ are separated by $\mathrm{FP}_{1}$, which can be seen more transparently in Fig. 9(b).

\section{RG analysis with $\mathbf{1} / \boldsymbol{n}$ corrections}

Upon incorporating $1 / n$ corrections, the RG flow equations [see Eqs. (30) and (31)] still support six fixed points. Even though the number of fixed points is impervious to the choice of $n$ (as long as $n \geq 4$ ), their locations receive nontrivial corrections for finite $n$ from the ones reported in Table III in the $n \rightarrow \infty$ limit. It is quite challenging to find the location of these fixed points analytically. Nevertheless, upon numerically locating the fixed points for various values of $n$, we can extract the functional dependence on $1 / n$. At least for large $n$ (namely, for $n \geq 8$ ), we find that the positions of all the fixed points are well approximated functions of $1 / n$, and they are tabulated in Table IV. All fixed points are located in the hyperplane $\lambda_{1}^{s}=0$. Therefore, this coupling constant does not change any outcome qualitatively or quantitatively.

The location of the six fixed points in the fourdimensional coupling constant space spanned by $\left(\lambda_{1}^{s}, \lambda_{3}^{s}\right.$, $\left.\lambda_{2}^{t}, \lambda_{3}^{t}\right)$ or $\left(U_{N}, U_{A}, W, X\right)$ are presented in Table IV. The nature of all the fixed points $\mathrm{FP}_{j}$ for $j=1, \ldots, 6$ remains unchanged from that discussed in the previous section after $1 / n$ corrections are included (see the last column of Table IV). From the leading order in $\epsilon$ and $1 / n$ expansion, we can also allude to some emergent quantum critical phenomena at the interacting QCPs (namely, $\mathrm{FP}_{2,3,4}$ ), describing continuous transitions from an ASM to various broken-symmetry phases. For example, the correlation length exponent at the interacting QCPs is given by $\nu^{-1}=\epsilon$ and, thus, for $n=2$ (physical situation), $\nu=2$, similar to the situation discussed previously for spinless fermions [see Eq. (29) for the definition of $\nu$ ]. The fact that the correlation length exponent is the same at all QCPs is, however, likely an artifact of the leading-order calculation.

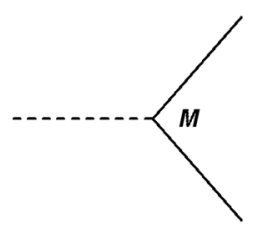

(a)

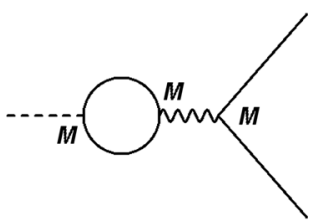

(b)

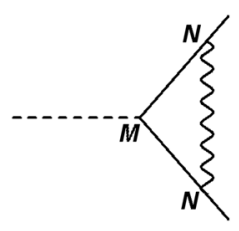

(c)
FIG. 10. (a) The bare vertex associated with a source term $\Psi \dagger M \Psi$ (particle-hole channel) or $\Psi M \Psi$ (particle-particle channel). $M$ and $N$ are four-dimensional Hermitian matrices [see Eqs. (33) and (35)]. Renormalization of the bare vertex (a) to the leading order in interaction couplings arises from diagrams (b) and (c). Note that there is no mixing of bilinear operators, since all transform differently under the symmetries; see Table II and Appendix C.

The residue of the quasiparticle pole of critical excitations (determined by the fermionic anomalous dimension $\eta_{\Psi}$ ) is expected to vanish smoothly, as these QCPs are approached from the ASM side of the transition. (To one-loop order, $\eta_{\Psi}=0$, so that a nontrivial fermionic anomalous dimension requires the computation of self-energy diagrams to two-loop order. We leave this exercise for future investigation.) On the other hand, if we subscribe to an appropriate order-parameter theory (also known as the Yukawa formalism), where the orderparameter or bosonic field is coupled with gapless fermions, a leading-order calculation yields nontrivial anomalous dimensions for both fermionic and bosonic fields [83]. The residue of the quasiparticle pole can serve as the order parameter in the semimetallic phase. The interacting QCPs describe strongly coupled nonFermi liquids, where the notion of sharp quasiparticle excitations becomes moot.

\section{SUSCEPTIBILITY OF SOURCE TERMS}

To pin the actual nature of the broken symmetry, we track the enhancement or suppression of two-point correlations amongst Hermitian fermion bilinears (order parameters). We do this by computing the flow equations for their corresponding source terms. The Hamiltonian including all of the source terms in the particle-hole or excitonic channel reads

$$
H_{s}^{p h}=-\int d^{2} \mathbf{r} \sum_{\mu=0}^{3} \Psi^{\dagger}\left[\Delta_{\mu}^{s} \sigma_{0} \tau_{\mu}+\Delta_{\mu}^{t} \vec{\sigma} \tau_{\mu}\right] \Psi .
$$

The physical interpretation of all fermion bilinears was summarized in Table II. The leading-order perturbative corrections to the source terms can be derived after computing the Feynman diagrams shown in Fig. 10. The resulting RG flow equations are 


$$
\begin{aligned}
& \frac{d \ln \Delta_{0}^{s}}{d l}=1+\frac{1}{4}\left(\lambda_{1}^{s}+\lambda_{3}^{s}+3 \lambda_{2}^{t}+3 \lambda_{3}^{t}\right) f_{2}(n) \equiv 1+\frac{1}{4}\left(U_{A}+X\right) f_{2}(n), \\
& \frac{d \ln \Delta_{1}^{s}}{d l}=1+\frac{1}{4}\left(3 \lambda_{1}^{s}+\lambda_{3}^{s}+3 \lambda_{2}^{s}+3 \lambda_{3}^{s}\right) f_{2}(n) \equiv 1+\frac{1}{4}\left(U_{A}-X\right) f_{2}(n), \\
& \frac{d \ln \Delta_{2}^{s}}{d l}=1+\frac{1}{2}\left(\lambda_{1}^{s}+\lambda_{3}^{s}-3 \lambda_{2}^{t}+3 \lambda_{3}^{t}\right)\left[f_{1}(n)-\frac{f_{2}(n)}{2}\right] \equiv 1+\frac{1}{4}\left(U_{A}+3 U_{N}+2 W\right)\left[f_{1}(n)-\frac{f_{2}(n)}{2}\right], \\
& \frac{d \ln \Delta_{3}^{s}}{d l}=1+\frac{1}{2}\left(\lambda_{1}^{s}+3 \lambda_{3}^{s}+3 \lambda_{2}^{t}-3 \lambda_{3}^{t}\right)\left[f_{1}(n)+\frac{f_{2}(n)}{2}\right] \equiv 1+\frac{1}{4}\left(U_{A}+3 U_{N}-2 W\right)\left[f_{1}(n)+\frac{f_{2}(n)}{2}\right], \\
& \frac{d \ln \Delta_{0}^{t}}{d l}=1+\frac{1}{4}\left(\lambda_{1}^{s}+\lambda_{3}^{s}-\lambda_{2}^{t}-\lambda_{3}^{t}\right) f_{2}(n) \equiv 1+\frac{1}{4}\left(U_{N}-X\right) f_{2}(n), \\
& \frac{d \ln \Delta_{1}^{t}}{d l}=1+\frac{1}{4}\left(-\lambda_{1}^{s}+\lambda_{3}^{s}-\lambda_{2}^{t}-\lambda_{3}^{t}\right) f_{2}(n) \equiv 1+\frac{1}{4}\left(U_{N}+X\right) f_{2}(n), \\
& \frac{d \ln \Delta_{2}^{t}}{d l}=1+\frac{1}{2}\left(\lambda_{1}^{s}+\lambda_{3}^{s}+5 \lambda_{2}^{t}-\lambda_{3}^{t}\right)\left[f_{1}(n)-\frac{f_{2}(n)}{2}\right] \equiv 1+\frac{1}{4}\left(U_{A}-U_{N}-2 W\right)\left[f_{1}(n)-\frac{f_{2}(n)}{2}\right], \\
& \frac{d \ln \Delta_{3}^{t}}{d l}=1+\frac{1}{2}\left(\lambda_{1}^{s}-\lambda_{3}^{s}-\lambda_{2}^{t}+5 \lambda_{3}^{t}\right)\left[f_{1}(n)-\frac{f_{2}(n)}{2}\right] \equiv 1+\frac{1}{4}\left(U_{A}-U_{N}+2 W\right)\left[f_{1}(n)+\frac{f_{2}(n)}{2}\right] .
\end{aligned}
$$

We also track the RG flow of the source terms corresponding to various local (momentum-independent or intra-unit-cell) superconducting orders, presented in Table II. The Hamiltonian capturing all local pairings assumes the form

$$
H_{s}^{p p}=-\int d^{2} \mathbf{r}\left[\Delta_{s} \Psi \sigma_{2} \tau_{3} \Psi+\Delta_{c h}^{1} \Psi \sigma_{2} \tau_{1} \Psi+\Delta_{c h}^{2} \Psi \sigma_{2} \tau_{0} \Psi+\Delta_{t}\left[\Psi \sigma_{3} \tau_{2} \Psi+\Psi \sigma_{0} \tau_{2} \Psi+\Psi \sigma_{1} \tau_{2} \Psi\right]\right]+\mathrm{H} . c .
$$

The leading RG flow equations for all the source terms associated with local pairings are given by

$$
\begin{aligned}
& \frac{d \ln \Delta_{s}}{d l}=1-\frac{1}{2}\left(\lambda_{1}^{s}-\lambda_{3}^{s}+3 \lambda_{2}^{t}+3 \lambda_{3}^{t}\right)\left[f_{1}(n)+\frac{f_{2}(n)}{2}\right] \equiv 1+\frac{1}{4}\left(-U_{A}+3 U_{N}\right)\left[f_{1}(n)+\frac{f_{2}(n)}{2}\right], \\
& \frac{d \ln \Delta_{c h}^{1}}{d l}=1+\frac{1}{4}\left(\lambda_{1}^{s}-\lambda_{3}^{s}-3 \lambda_{2}^{t}+3 \lambda_{3}^{t}\right) f_{2}(n) \equiv 1+\frac{1}{4}(W-X) f_{2}(n), \\
& \frac{d \ln \Delta_{c h}^{2}}{d l}=1-\frac{1}{4}\left(\lambda_{1}^{s}+\lambda_{3}^{s}+3 \lambda_{2}^{t}-3 \lambda_{3}^{t}\right) f_{2}(n) \equiv 1+\frac{1}{4}(W+X) f_{2}(n), \\
& \frac{d \ln \Delta_{t}}{d l}=1-\frac{1}{2}\left(\lambda_{1}^{s}+\lambda_{3}^{s}+\lambda_{2}^{t}+\lambda_{3}^{t}\right)\left[f_{1}(n)-\frac{f_{2}(n)}{2}\right] \equiv 1-\frac{1}{4}\left(U_{A}+U_{N}\right)\left[f_{1}(n)-\frac{f_{2}(n)}{2}\right]
\end{aligned}
$$

The quantities on the right-hand side of Eqs. (34) and (36) are the scaling dimensions of the corresponding source terms. The scaling dimensions associated to all fermionic bilinear source terms at the three interacting QCPs (namely, $\mathrm{FP}_{2}, \mathrm{FP}_{3}$, and $\mathrm{FP}_{4}$ ) are displayed in Table V. If the source term has scaling dimension $\mathrm{y}_{\mathrm{s}}$, the anomalous dimension of the associated fermion bilinear $\mathrm{y}_{\mathrm{b}}$ is given by

$$
\mathrm{y}_{\mathrm{b}}=2+1 / n-\mathrm{y}_{\mathrm{s}}
$$

using the fact that the operator and coupling constant scaling dimensions add to the scaling dimension of spacetime [84]. The latter is equal to $2+1 / n$, according to Sec. II A. Since $y_{b}$ controls the decay of correlations (in the $x$ direction) of the bilinear two-point function at the quantum critical point [84], a larger source dimension $y_{s}$ means stronger correlations (smaller $\mathrm{y}_{\mathrm{b}}$, slower decay).

\section{A. Scaling dimensions of fermion bilinears: Spin and pseudospin symmetries}

In Table $\mathrm{V}$, the bilinears previously cataloged in Table II are identified with $8 \times 8$ matrices, obtained by combining physical spin $(\sigma)$ and sublattice/orbital $(\tau)$ with particle-hole $(\eta)$ degrees of freedom. The matrix representations of the various fermionic bilinears in Table $\mathrm{V}$ are obtained in the eight-component Nambu doubled spinor basis: 
TABLE V. The scaling dimensions (SDs) of the source term coupling constants conjugate to various fermionic bilinears at three interacting quantum critical points (QCPs), $\mathrm{FP}_{2}, \mathrm{FP}_{3}$, and $\mathrm{FP}_{4}$, possessing only one unstable direction in the $\hat{\Delta}(l)=0$ hyperplane (see text for details). Note that, at each QCP, we display the largest SD in red and subdominant SD in blue. As we take $n \rightarrow \infty$ (strict onedimensional system), the red and blue channels possess exactly equal SDs and constitute an $\mathrm{O}(4)$ vector. However, such $\mathrm{O}(4)$ symmetry gets lifted once the $1 / n$ corrections are systematically accounted for. Upon incorporating the $1 / n$ corrections, the SD for one of the fully gapped (true mass) orders (CDW, AFM, s-wave pairing) for the two-dimensional anisotropic semimetal is largest at each QCP. We, therefore, anticipate that $\mathrm{FP}_{2}, \mathrm{FP}_{3}$, and $\mathrm{FP}_{4}$, respectively, nucleate $\mathrm{CDW} / s$-wave pairing, $\mathrm{CDW}$ order, and Néel antiferromagnetism. Here, all fermionic bilinears are presented in the Nambu-doubled spinor basis introduced in Eq. (37) (see also Appendix C), and the Pauli matrices $\eta_{\mu}=\left\{\eta_{0}, \eta_{1}, \eta_{2}, \eta_{3}\right\}$ operate on the Nambu index. The column PS indicates bilinears that transform together under pseudospin SU(2) rotations; see Fig. 11. The other fermion bilinears transform as scalars under pseudospin rotation.

\begin{tabular}{|c|c|c|c|c|c|c|}
\hline $\begin{array}{l}\text { Source } \\
\text { term }\end{array}$ & PS & Physical meaning & $\begin{array}{c}\text { Matrix } \\
\text { representation }\end{array}$ & $\mathrm{FP}_{2}$ & $\mathrm{FP}_{3}$ & $\mathrm{FP}_{4}$ \\
\hline$\Delta_{0}^{s}$ & III & Chemical potential or density & $\eta_{3} \sigma_{0} \tau_{0}$ & 1 & $1+[\epsilon /(4 n)]$ & $1+[\epsilon /(4 n)]$ \\
\hline$\Delta_{1}^{s}$ & IV & Abelian current in $x$ direction & $\eta_{0} \sigma_{0} \tau_{1}$ & 1 & $1+[\epsilon /(4 n)]$ & $1+[\epsilon /(4 n)]$ \\
\hline$\Delta_{2}^{s}$ & & Anisotropy parameter & $\eta_{3} \sigma_{0} \tau_{2}$ & $1+\left[\frac{3}{4}-\frac{0.275}{n}\right] \epsilon$ & $1+\left[-\frac{1}{4}+(0.5 / n)\right] \epsilon$ & $1+\left[\frac{3}{4}-\frac{0.85}{n}\right] \epsilon$ \\
\hline$\Delta_{3}^{s}$ & I & Charge density wave & $\eta_{3} \sigma_{0} \tau_{3}$ & $1+\left[\frac{3}{4}+\frac{0.025}{n}\right] \epsilon$ & $1+\left[\frac{3}{4}+\frac{1.02}{n}\right] \epsilon$ & $1+\left[-\frac{1}{4}-(0.35 / n)\right] \epsilon$ \\
\hline$\Delta_{0}^{t}$ & & Magnetization & $\eta_{0}\left(\sigma_{1}, \sigma_{2}, \sigma_{3}\right) \tau_{0}$ & $1+[\epsilon /(4 n)]$ & 1 & 1 \\
\hline$\Delta_{1}^{t}$ & & Abelian spin current along $x$ & $\eta_{3}\left(\sigma_{1}, \sigma_{2}, \sigma_{3}\right) \tau_{1}$ & $1+[\epsilon /(4 n)]$ & 1 & 1 \\
\hline$\Delta_{2}^{t}$ & II & Spin bond-density wave & $\eta_{0}\left(\sigma_{1}, \sigma_{2}, \sigma_{3}\right) \tau_{2}$ & $1+\left[-\frac{1}{4}+(0.092 / n)\right] \epsilon$ & $1+\left[\frac{3}{4}-\frac{0.26}{n}\right] \epsilon$ & $1+\left[-\frac{1}{4}+(0.28 / n)\right] \epsilon$ \\
\hline$\Delta_{3}^{t}$ & & Antiferromagnet or Néel & $\eta_{0}\left(\sigma_{1}, \sigma_{2}, \sigma_{3}\right) \tau_{3}$ & $1+\left[-\frac{1}{4}+(0.292 / n)\right] \epsilon$ & $1+\left[-\frac{1}{4}-(0.29 / n)\right] \epsilon$ & $1+\left[\frac{3}{4}+\frac{0.286}{n}\right] \epsilon$ \\
\hline$\Delta_{s}$ & I & $s$-wave pairing & $\left(\eta_{1}, \eta_{2}\right) \sigma_{0} \tau_{0}$ & $1+\left[\frac{3}{4}+\frac{0.025}{n}\right] \epsilon$ & $1+\left[-\frac{1}{4}+(0.165 / n)\right] \epsilon$ & $1+\left[-\frac{1}{4}-(0.35 / n)\right] \epsilon$ \\
\hline$\Delta_{c h}^{1}$ & IV & Chiral singlet pairing ${ }_{1}$ & $\left(\eta_{1}, \eta_{2}\right) \sigma_{0} \tau_{2}$ & 1 & $1-[\epsilon /(4 n)]$ & $1+[\epsilon /(4 n)]$ \\
\hline$\Delta_{c h}^{2}$ & III & Chiral singlet pairing $_{2}$ & $\left(\eta_{1}, \eta_{2}\right) \sigma_{0} \tau_{3}$ & 1 & $1-[\epsilon /(4 n)]$ & $1+[\epsilon /(4 n)]$ \\
\hline$\underline{\Delta_{t}}$ & II & Triplet pairing & $\left(\eta_{1}, \eta_{2}\right)\left(\sigma_{1}, \sigma_{2}, \sigma_{3}\right) \tau_{1}$ & $1+\left[-\frac{1}{4}+(0.092 / n)\right] \epsilon$ & $1+\left[-\frac{1}{4}-(0.115 / n)\right] \epsilon$ & $1+\left[-\frac{1}{4}+(0.28 / n)\right] \epsilon$ \\
\hline
\end{tabular}

$$
\begin{aligned}
\Psi_{N}^{\top}= & {\left[c_{A, \mathbf{k}, \uparrow}^{\dagger}, c_{B, \mathbf{k}, \uparrow}^{\dagger}, c_{A, \mathbf{k}, \downarrow}^{\dagger}, c_{B, \mathbf{k}, \downarrow}^{\dagger},\right.} \\
& \left.c_{A,-\mathbf{k}, \downarrow},-c_{B,-\mathbf{k}, \downarrow},-c_{A,-\mathbf{k}, \uparrow}, c_{B,-\mathbf{k}, \uparrow}\right] .
\end{aligned}
$$

In this basis, the three generators of physical spin $(\vec{S})$ and pseudospin $(\overrightarrow{P S})[44-46,85]$ symmetry are given by

$\vec{S}=\eta_{0}\left(\sigma_{1}, \sigma_{2}, \sigma_{3}\right) \tau_{0}, \quad \overrightarrow{P S}=\left(\eta_{1} \sigma_{0} \tau_{3}, \eta_{2} \sigma_{0} \tau_{3}, \eta_{3} \sigma_{0} \tau_{0}\right)$

Note that the pseudospin generators do not involve genuine spin degrees of freedom, and one of its generators, namely, $P S_{3}$, is the charge density operator. The remaining two generators of pseudospin symmetry are the real and imaginary components of the chiral singlet pairing . See Appendix $\mathrm{C}$ for symmetries in the Nambu basis.

We now discuss the results. First, we consider the 1D $(n \rightarrow \infty)$ limit, as a check. Then, Eq. (26) implies that we should set $f_{1}(n)=1$ and $f_{2}(n)=0$ in Eqs. (34) and (36). We see that the charge bond density wave/anisotropy, CDW, spin bond-density wave, and antiferromagnet source fields $\Delta_{2,3}^{s, t}$ receive an anomalous correction $+U_{A} / 4$, while the singlet and triplet SC terms $\Delta_{s, t}$ receive the anomalous correction $-U_{A} / 4$, when $U_{N}=W=X=0$. The remaining terms receive no corrections in this limit. This is consistent with the predictions of bosonization, summarized in Table VII and using the Luttinger parameter in Eq. (D4).

Next, note that at $\mathrm{FP}_{2}$, the scaling dimensions for CDW and $s$-wave pairing are largest and exactly equal, reflecting the underlying pseudospin $\mathrm{SU}(2)$ symmetry among these two orders; see (I) in Fig. 11. Since the bipartite lattice Hubbard Hamiltonian possesses pseudospin SU(2) symmetry at half filling [44-46], we expect that $\mathrm{FP}_{2}$ can be accessed by tuning the strength of on-site attraction (see Sec. VIII). On the other hand, the scaling dimension for CDW order is largest amongst all possible orders at the interacting $\mathrm{QCP} \mathrm{FP}_{3}$. Hence, it is natural to anticipate that repulsive $\mathrm{NN}$ interaction in the strained honeycomb model can induce the transition across this fixed point. By contrast, the AFM order possesses the largest scaling dimension at the interacting $\mathrm{QCP} \mathrm{FP}_{4}$, indicating that the onset of AFM for strong repulsive Hubbard interaction is controlled by this fixed point. In the following section, we will substantiate these observations from the phase diagram of an extended Hubbard model. The fact that a twodimensional ASM supports only six mass matrices representing CDW (1), AFM (3), and $s$-wave pairing (2), where the quantities in the parentheses denote the number of matrices required to define a specific order parameter (see 

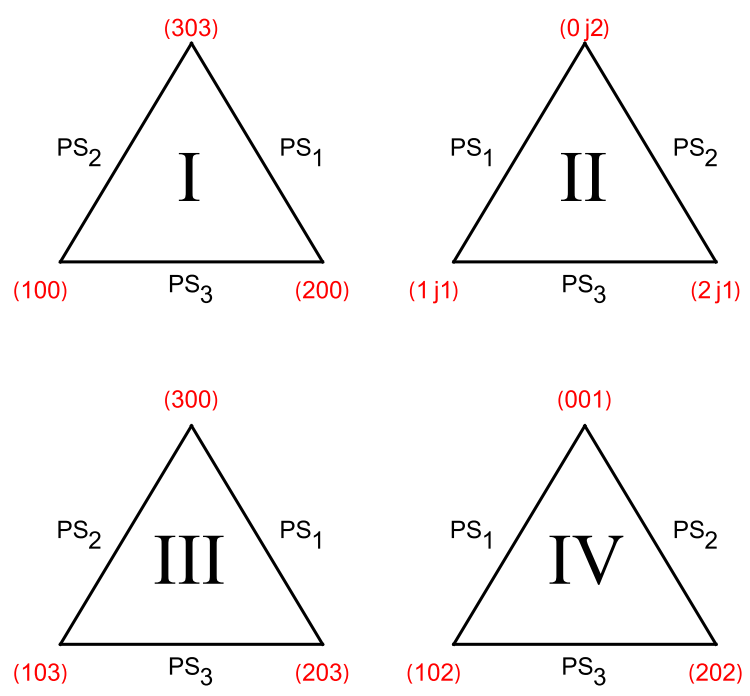

FIG. 11. Four sets of three mutually anticommuting fermionic bilinears, denoted by $(i k l) \equiv \eta_{i} \sigma_{k} \tau_{l}$, sitting at the vertices of four triangles (see Table V). Each triangle demonstrates the SU(2) pseudospin rotation among (I) the charge density wave and two (real and imaginary) components of $s$-wave pairing; (II) the spin bond-density wave and two components (real and imaginary) of triplet pairing with a specific spin orientation $(j=1,2,3)$; (III) the charge density and the components (real and imaginary) of chiral singlet pairing ${ }_{2}$; and (IV) the $x$-charge current and the components (real and imaginary) of chiral singlet pairing $_{1}$. The pseudospin generators ( $\mathrm{PS}_{j}$ with $j=1,2,3$ ) [Eq. (38)] correspond to (III). Any arm of a triangle represents the rotation between two bilinears, sitting at two corresponding vertices, by a specific generator.

Table V), can also be established from the Clifford algebra of real matrices, as shown in Appendix G.

The pseudospin symmetry between $s$-wave pairing and CDW order at $\mathrm{FP}_{2}$ extends to three other bilinear "triads." Each triad contains three bilinears that transform in the pseudospin triplet representation, as explained in Fig. 11. At $\mathrm{FP}_{2}$ and $\mathrm{FP}_{4}$, the bilinears within each triad share a common scaling dimension, indicating that pseudospin $\mathrm{SU}(2)$ symmetry is emergent at both of these QCPs; see (II), (III), and (IV) in Fig. 11. This suggests that $\mathrm{FP}_{2,4}$ are realized via attractive or repulsive on-site Hubbard interactions in the strained honeycomb lattice model, as we confirm in Sec. VIII. By contrast, pseudospin symmetry is explicitly broken at $\mathrm{FP}_{3}$. The perturbative restoration of pseudospin symmetry at interacting QCPs that can be accessed by tuning the Hubbard interaction serves as a good anchoring ground of the analysis [86].

\section{B. Identification of broken-symmetry phases}

The strategy for identifying the actual nature of the broken symmetry at strong coupling is the following. We simultaneously run the flow of the interaction coupling constants and of the source terms. When interactions are weak, all of them flow back to zero under coarse graining, and none of the source terms diverges, indicating the stability of the ASM for weak enough interactions.

As we keep increasing the strength of interaction, beyond a critical strength, at least one of the interaction coupling constants diverges. At the same time, at least one of the source terms diverges. The channel (say, $\Delta_{\mu}$ ) that diverges fastest determines the pattern of spontaneous symmetry breaking and the resulting phase is characterized with the order parameter $\Delta_{\mu} \neq 0$. Following this strategy, we present various cuts of the phase diagram of the interacting ASM in Fig. 12. We follow the same strategy to arrive at the phase diagram of an extended Hubbard model, discussed in the next section.

\section{1D physics in 2D?}

The $1 / n$ corrections to the interaction $\mathrm{RG}$ flow in Eq. (31) proportional to $f_{2}(n) \operatorname{mix}$ the spin $\left(U_{N}\right)$ and charge $\left(U_{A}, W, X\right)$ sector interaction coupling constants. This suggests that spin-charge separation is completely destroyed for any finite $n$; the one-dimensional physics does not survive once quantum fluctuations in 2D are incorporated. On the other hand, the pseudospin-SU(2)symmetric fixed points $\mathrm{FP}_{2}$ and $\mathrm{FP}_{4}$ are reminiscent of the 1D spin-charge separated Luther-Emery liquid and SDW phases, respectively. Both the 1D phases and 2D fixed points sit on the precipice of long-range $\mathrm{CDW} / s$-wave pairing or antiferromagnetic order, and both are expected to lack well-defined quasiparticles. It would therefore be extremely interesting to look for remnants of 1D physics at these fixed points upon incorporating higher-order corrections (two loops and beyond).

\section{EXTENDED HUBBARD MODEL}

We now focus on the phase diagram of the extended Hubbard model in 1D and for the ASM in two spatial dimensions. We discuss these two cases separately.

\section{A. One dimension}

The extended Hubbard interaction in 1D is

$$
\begin{aligned}
H_{\mathrm{UV}}^{1 D}= & U \sum_{j} c_{j, \uparrow}^{\dagger} c_{j, \uparrow} c_{j, \downarrow}^{\dagger} c_{j, \downarrow} \\
& +V \sum_{j, \sigma=\uparrow, \downarrow} c_{j, \sigma}^{\dagger} c_{j, \sigma} c_{j+1, \sigma}^{\dagger} c_{j+1, \sigma} .
\end{aligned}
$$

Here $j$ is the 1D lattice site index. Since the single-band 1D system supports two Dirac points at momentum $k_{x}= \pm k_{F}$, we can expand the lattice fermion operator in terms of the low energy Fourier modes according to

$$
c_{\sigma}=e^{i k_{F} x} R_{\sigma}+e^{-i k_{F} x} L_{\sigma},
$$

where $R_{\sigma}$ and $L_{\sigma}$ are right- and left-mover operators for modes at $\pm k_{F}$, with spin projection $\sigma=\uparrow, \downarrow$. At half 


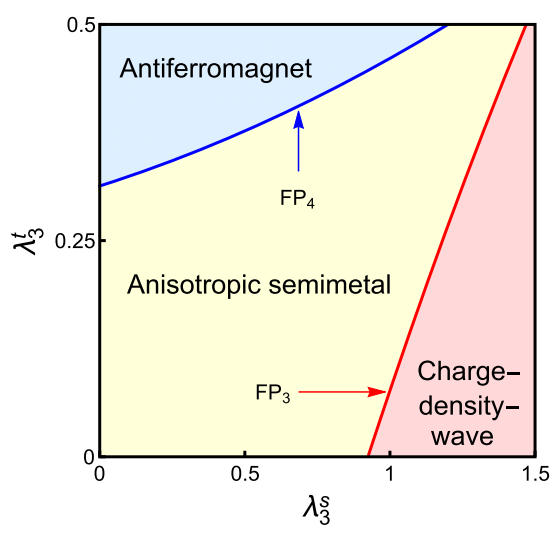

(a)

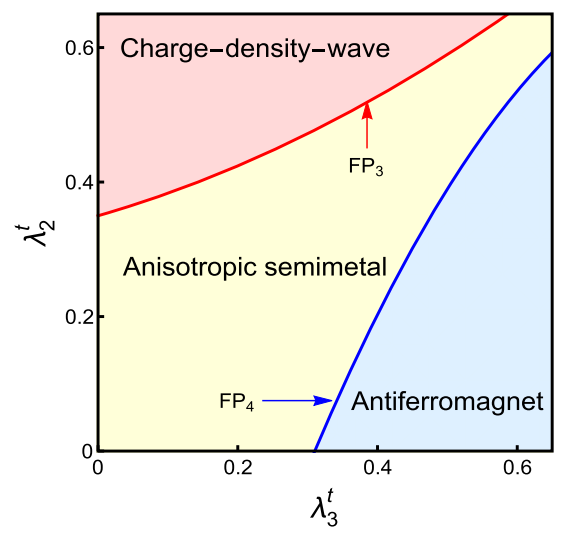

(b)

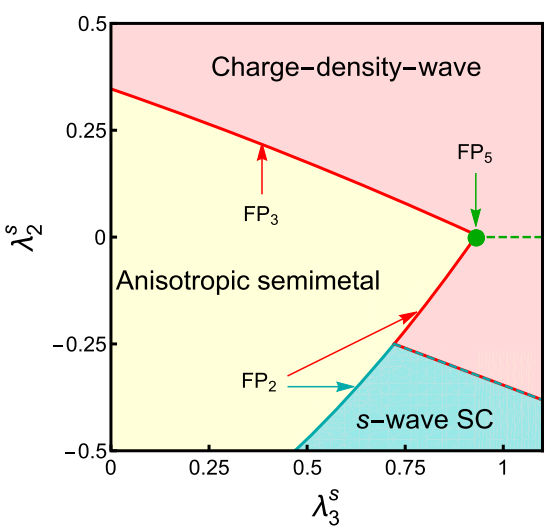

(c)

FIG. 12. Various cuts of the phase diagram for the spin-1/2 interacting ASM, residing at the QCP between the two-dimensional Dirac semimetal and the band insulator. These phase diagrams are obtained for $n=10$, which parametrically keeps the quantum fluctuations beyond one dimension small. All coupling constants are dimensionless and measured in units of $\epsilon$. Various fixed points $\left(\mathrm{FP}_{j}\right.$ 's; see Table IV) controlling the continuous quantum phase transition from the ASM to different broken-symmetry phases are displayed in each cut of the phase diagram. Note that $\mathrm{FP}_{2,3,4}$ are interacting QCPs, while $\mathrm{FP}_{5}$ is an interacting bicritical point [appearing at the point of inflection in panel (c), the green dot]. Along the dashed red/cyan line in (c), the CDW and $s$-wave SC simultaneously display long-range order, because of the pseudospin SU(2) symmetry. Below (above) this line, only $s$-wave SC (CDW) order is realized as true long-ranged order. The dashed green line is a crossover boundary, across which the short-range correlations of $s$-wave pairing vanish smoothly from bottom to top. While extracting the phase diagram in each two-dimensional coupling constant space, we set the bare value of the other two couplings to be zero.

filling, the extended Hubbard interaction from Eq. (39) leads to the continuum form

$H_{\mathrm{UV}}^{1 D}=\int d x\left[U_{N} \mathcal{O}_{N}+U_{A} \mathcal{O}_{A}+\frac{W}{2}\left(\mathcal{O}_{W}+\overline{\mathcal{O}}_{W}\right)+X \mathcal{O}_{X}\right]$,

where the interaction operators are defined in Eq. (D2). The initial or bare conditions (values of coupling constants at RG time $l=0$ ) for the 1D extended Hubbard model are

$$
\begin{aligned}
U_{N} & =V-\frac{1}{2} U, & U_{A} & =\frac{1}{2} U+3 V, \\
W & =\frac{1}{2} U-V, & X & =\frac{1}{2} U+V .
\end{aligned}
$$

The standard 1D RG flow equations in Eq. (32) (with $\epsilon=0$ ) then determine the phase diagram of the onedimensional extended Hubbard model, displayed in Fig. 4(a) [42]. The diagonal phase boundaries in Fig. 4(a) are determined by $U= \pm 2 \mathrm{~V}$, based on the lowest-order Kosterlitz-Thouless equations [Eq. (32) with $\epsilon=0$ ].

\section{B. Two-dimensional ASM}

Since the Dirac-semimetal to band-insulator quantum phase transition takes place when two Dirac points with opposite vorticity merge at a high symmetry point in the Brillouin zone, the ASM in two dimensions lacks any valley (chiral) degeneracy. Thus, we can simply identify the sublattice density operators with

$$
\begin{aligned}
& n_{\uparrow / \downarrow}(\vec{A})=\frac{1}{4} \Psi^{\dagger}\left(\sigma_{0} \pm \sigma_{3}\right)\left(\tau_{0}+\tau_{3}\right) \Psi, \\
& n_{\uparrow / \downarrow}(\vec{B})=\frac{1}{4} \Psi^{\dagger}\left(\sigma_{0} \pm \sigma_{3}\right)\left(\tau_{0}-\tau_{3}\right) \Psi .
\end{aligned}
$$

Taking the continuum limit of the extended Hubbard Hamiltonian from Eq. (8), we obtain the interaction Hamiltonian $H_{\text {int }}$ in the form of Eq. (16). We thereby determine the initial conditions for the extended Hubbard model in the two-dimensional ASM,

$$
\begin{aligned}
g_{1}^{s} & =-\frac{3}{8} V-\frac{1}{4} U, & g_{3}^{s} & =\frac{3}{4} V, \\
g_{2}^{t} & =\frac{1}{8} V+\frac{1}{12} U, & g_{3}^{t} & =\frac{1}{4} V+\frac{1}{6} U .
\end{aligned}
$$

In this notation, $X>0$ corresponds to repulsive interaction, while attractive interaction is realized for $X<0$, where $X=U, V$.

We follow the strategy described in Sec. VII B for various values of $U$ and $V$ (both attractive and repulsive interactions) to arrive at the phase diagram of the interacting ASM in the $U-V$ plane. Our result is shown in Fig. 4(b). For strong enough on-site repulsion $U>0$, the susceptibility in the AFM channel displays the leading divergence, as shown in Fig. 13(a), indicating the onset of collinear antiferromagnetic (Néel) order on the strained honeycomb lattice. On the other hand, for sufficiently strong attractive $U<0$, the $s$-wave and CDW channels diverge in an exactly degenerate fashion, as shown in Fig. 13(b), suggesting simultaneous nucleation of these two orders. Thus, the onset of long-range 


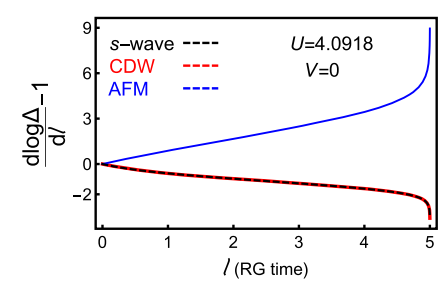

(a)

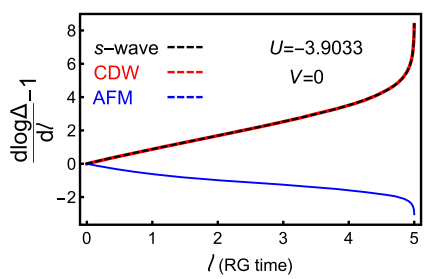

(b)

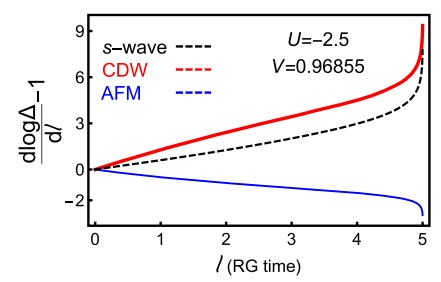

(c)

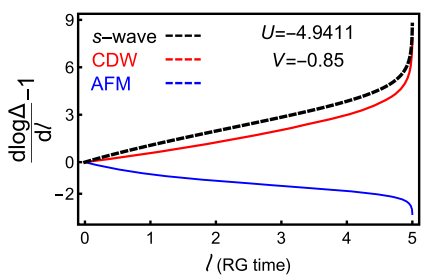

(d)

FIG. 13. Renormalization group flow of the source terms in the antiferromagnet (blue), charge density wave (red), and singlet $s$-wave pairing (black) channels for various choices for the bare strength (at $l=0$ ) of on-site $(U)$ and nearest-neighbor $(V)$ interactions in a uniaxially strained honeycomb lattice. The strengths of both interactions are quoted in dimensionless units. The onset of an order parameter is associated with the divergence $(\rightarrow+\infty)$ of the corresponding source term. The flow of the source terms shows that strong repulsive $U>0$ causes formation of antiferromagnetic order [see panel (a)]. By contrast, for a strong attractive $U<0$, diverging susceptibilities in the CDW and $s$-wave pairing channels are exactly degenerate, as guaranteed by the pseudospin SU(2) symmetry of the system with $V=0$ (see Sec. VII A). These two orders nucleate simultaneously in the strong coupling regime [see panel (b)]. The addition of the nearest-neighbor interaction $V$ breaks such exact degeneracy. In particular, $V>0(V<0)$ favors the nucleation of the CDW ( $s$-wave pairing), as shown in panel (c) [panel (d)]. The resulting phase diagram in the $U$ - $V$ plane is shown in Fig. 4(b). For the clarity of presentation, we here do not present the RG flow of the remaining channels. We have verified that, in the entire phase diagram, only one of these three channels displays the leading divergence at strong coupling.

order for strong repulsive and attractive Hubbard interaction takes place through spontaneous breaking of the SU(2) spin and pseudospin symmetries, respectively. However, the exact (microscopic) pseudospin $\mathrm{SU}(2)$ symmetry gets destroyed for any $V \neq 0$. In particular, for $V>0(V<0)$ only the susceptibility of the CDW ( $s$-wave pairing) diverges, as shown in Fig. 13(c) [Fig. 13(d)].

Finally, we discuss the role of various interacting QCPs, discussed in Sec. VI, on the phase diagram of the extended Hubbard model. The fixed point controlling the transition into the broken-symmetry phase can be identified by scrutinizing the RG flow trajectories of the interaction coupling constants. The continuous transition between the ASM and AFM for repulsive $U>0$ is controlled by the interacting $\mathrm{QCP} \mathrm{FP}_{4}$, while the transition into a coexisting phase of $s$-wave pairing and CDW order $(U<0, V=0)$ takes place through the interacting $\mathrm{QCP} \mathrm{FP}_{2}$. The ASM$\mathrm{CDW}$ continuous transition driven by $\mathrm{NN}$ repulsion is governed by the interacting QCP $\mathrm{FP}_{3}$. Notice that, for $V>0$, as we keep increasing the strength of attractive onsite interaction, such that the broken-symmetry phase at strong coupling is always the CDW, the fixed point that controls the ASM-CDW continuous transition switches from $\mathrm{FP}_{3}$ to $\mathrm{FP}_{2}$ for an intermediate strength of negative Hubbard- $U$. At this point, the ASM-CDW phase boundary displays a cusp, as shown with a dot in Fig. 4(b), and the ASM-CDW transition at this point is controlled by the bicritical point $\mathrm{FP}_{5}$. Since such a bicritical point is accessed by holding one of its two unstable directions fixed, the ASM-CDW transition is always continuous.

In the phase diagram of the extended Hubbard model for the two-dimensional ASM [see Fig. 4(b)], we show a dashed green line for repulsive NN but attractive on-site interaction. We now discuss the physical significance of this line. The scaling dimensions for $s$-wave pairing and
$\mathrm{CDW}$ order source terms at the interacting fixed points $\mathrm{FP}_{2}$, $\mathrm{FP}_{5}$, and $\mathrm{FP}_{3}$, respectively, read as

$$
\begin{aligned}
& \mathrm{y}_{\text {s-wave }}=1+\left\{\begin{array}{llc}
\frac{3}{4}+\frac{0.025}{n}, & \frac{1}{2}-\frac{0.5245}{n}, & -\frac{1}{4}+\frac{0.165}{n} \\
\mathrm{FP}_{2} & \mathrm{FP}_{5} & \mathrm{FP}_{3}
\end{array}\right\} \epsilon \\
& \mathrm{y}_{\mathrm{CDW}}=1+\left\{\begin{array}{llc}
\frac{3}{4}+\frac{0.025}{n}, & \frac{3}{2}-\frac{0.2625}{n}, & \frac{3}{4}+\frac{1.02}{n} \\
\mathrm{FP}_{2} & \mathrm{FP}_{5} & \mathrm{FP}_{3}
\end{array}\right\} \epsilon .
\end{aligned}
$$

While the scaling dimensions for these two orders are exactly equal at $\mathrm{FP}_{2}$, at the two other fixed points the scaling dimension for the CDW is always bigger than that for $s$-wave SC. Consequently, we determine that CDW order is favored for strong attractive $U<0$ and repulsive $V>0$. Also note that, starting from $V=0$ and attractive $U<0$, if we increase $V$ along the ASM-CDW phase boundary [see the red line in Fig. 4(b)], the transition is first controlled by $\mathrm{FP}_{2}$ (for weak $V>0$ ), then $\mathrm{FP}_{5}$ (for a specific strength of $V$, indicated by the green dot), and, finally, by $\mathrm{FP}_{3}$ (stronger $\mathrm{NN}$ repulsion $V>0$ ). The scaling dimension of the $s$-wave pairing source term decreases monotonically from $\mathrm{FP}_{2}$ to $\mathrm{FP}_{5}$ to $\mathrm{FP}_{3}$ for any $n \geq 2$. Therefore, in the entire regime of the CDW phase [the red shaded region in Fig. 4(b)], $s$-wave pairing can only be found as a short-range order, without any global long-range order. Furthermore, the coherence length $(\xi)$ of such local $s$-wave pairing decreases monotonically as we keep increasing $V>0$ (keeping $U<0$ ) and, across the dashed green line, $\xi$ vanishes smoothly. Therefore, above the dashed green line in Fig. 4(b), local short-range $s$-wave pairing loses its support. It must be noted that there is no genuine phase transition across the green line; it only represents a crossover boundary.

Our RG analysis leads to a fairly complete phase diagram of the extended Hubbard model in the uniaxially 
strained honeycomb lattice, residing at or very close to the Dirac-semimetal to band-insulator QCP. We identified the role of each of the six fixed points (Table IV) in the phase diagram of a simple microscopic interacting model. With recent progress in controlling the strength of electronic interactions in optical honeycomb lattices for ultracold fermionic atoms $[13,14]$ and the realization of an ultracoldatom Fermi-Hubbard antiferromagnet [87], as well as quantum Monte Carlo simulation with on-site or NN interaction [57-64], we believe that the phase diagram of the extended Hubbard model can be established (at least partially) in numerical simulation and/or experiments.

The existence of the three interacting critical points $\mathrm{FP}_{2,3,4}$ can be argued to survive beyond perturbation theory. For strong repulsive Hubbard interactions on a bipartite lattice in 2D, SU (2) spin rotational symmetry is broken and the system enters into the AFM phase, while, for strong attractive Hubbard interactions, pseudospin SU(2) symmetry is lifted, leading to simultaneous nucleation of $s$-wave pairing and CDW. By contrast, for strong repulsive nearest-neighbor interactions on a bipartite lattice, the system enters the CDW phase, where discrete $Z_{2}$ sublattice symmetry is broken. The existence of these phases at sufficiently strong interaction can be anticipated for the strained honeycomb lattice model, which is free of frustration for all of the above orderings. However, because of the vanishing DoS in the ASM $[\varrho(E) \sim \sqrt{E}]$, all the broken-symmetry phases are realized only beyond a critical threshold of interaction and, thus, through a quantum phase transition. We expect that the transitions are continuous in the strained honeycomb lattice extended Hubbard model and, thus, controlled by QCPs. We note that quantum Monte Carlo simulation in the Dirac system (isotropic honeycomb lattice model) also indicates continuous transitions through various QCPs [57-64]. Thus, future numerical works can shed light on the nonperturbative nature of these transitions, which are accessed here from leading-order $\epsilon$ and $1 / n$ expansions.

\section{DISCUSSION AND CONCLUSION}

In this paper, we discuss the effect of generic short-range interactions at the quantum critical point separating a twodimensional topological Dirac semimetal and a symmetrypreserving band insulator. The critical excitations separating these two phases constitute an anisotropic semimetal. The quasiparticle spectra in the anisotropic semimetal display both linear and quadratic dependence on momenta along two mutually orthogonal directions. Consequently, the quantum critical regime in the noninteracting system displays peculiar power-law scaling of thermodynamic and transport quantities that is distinct from their counterparts in a Dirac semimetal or a band insulator, as shown in Table I.

Because of the vanishing density of states $[\varrho(E) \sim \sqrt{E}]$, the critical point separating the Dirac semimetal from the band insulator remains stable against sufficiently weak short-range electron-electron interactions. However, at strong interaction, this direct transition either (i) becomes a fluctuation-driven first-order transition (see Sec. VA) or (ii) gets avoided by an intervening broken-symmetry phase (see Sec. VI). Using RG analysis, we identify charge density wave, antiferromagnet, and spin singlet $s$-wave pairing as the dominant channels of symmetry breaking for the interacting model containing generic short-range interactions that, for example, encompass the extended Hubbard model; see the phase diagrams in Figs. 4(b) and 12.

We demonstrate that (see Sec. VI) the RG can be controlled by expanding about one dimension, for which short-range interactions are marginal at tree level. We recover standard results for $1 \mathrm{D}$ systems, such as the absence of corrections for a U(1) current-current (Luttinger) perturbation, as well as spin-charge separation and Kosterlitz-Thouless transitions for spin-1/2 electrons. From the leading-order calculation, we find that the correlation length exponent at all the interacting multicritical points describing a continuous quantum phase transition from anisotropic semimetal to various brokensymmetry phases is $\nu=2$, which is strikingly different from the ones across a Dirac-semimetal to brokensymmetry phase transition for which $\nu \approx 1$, as predicted in field theoretic works $[34,66,80-82,86,88,89]$, and has also been established numerically [58-61,63,64].

Throughout, we have neglected the long-range tail of the Coulomb interaction and focused only on its short-range pieces. Recently it has been proposed that infinitesimally weak long-range Coulomb interaction can cause an instability of the critical excitations and drive the system toward the formation of an infrared-stable non-Fermi liquid phase $[6,7]$. In the future, it will be interesting to study the interplay of non-Fermi liquid and broken-symmetry phases. However, in an optical honeycomb lattice (composed of neutral atoms), our study should provide a fairly complete picture of the phase diagram for the interacting uniaxially strained honeycomb lattice. Our results also apply to solid state compounds with screened Coulomb interactions (because of, e.g., an external gate).

The RG framework developed here (combined $\epsilon$ and $1 / n$ expansion) can be applied to many different systems where quasiparticle excitations possess anisotropy, such as a three-dimensional general Weyl semimetal constituted by monopoles and antimonopoles of strength $\mathcal{N}$ [65], with $\mathcal{N}=1,2,3$ in a crystalline environment [90-92]. In addition, our formalism (with minor modifications) can also be subscribed to address the effects of quenched disorder at the Weyl semimetal-band insulator quantum critical point and possible onset of a metallic phase at strong disorder through a continuous quantum phase transition across a multicritical point [93]. Furthermore, our formalism can also shed light on the effects of 
electronic interaction in a two-dimensional Dirac semimetal. At the cost of the in-plane rotational symmetry $\left(v_{x} \neq v_{y}\right.$, where $v_{j}$ is the Fermi velocity of massless Dirac fermions in the $j$ direction), which can be achieved by applying a weak uniaxial strain in monolayer graphene (so that the system is sufficiently far from the Diracsemimetal to band-insulator quantum critical point), we can generalize the proposed $\epsilon$ and $1 / n$ expansion to address competing orders, emergent quantum critical phenomena, and the global phase diagram of a $2 \mathrm{D}$ Dirac system (with the caveat that $n$ can only be an odd integer). The interacting theory for a slightly anisotropic Dirac semimetal (with two valleys) is described by four (eight) independent coupling constants for spinless (spinful) fermions. This exercise will allow us to investigate an intriguing competition among various ordered phases, such as antiferromagnet, valence-bond-solid, charge-density-wave, singlet, and triplet superconductors as well as topological quantum anomalous/spin Hall insulators, in a controlled renormalization group framework [8]. We leave this problem for future work [94].

Finally, we point out that the dispersion of the ASM studied here closely resembles that of quasiparticles near hot spots of the Fermi surface in a two-dimensional square lattice system [95]. Recently, there has been a surge of theoretical works geared toward understanding the effects of strong forward scattering interactions and quantum critical phenomena within the hot spot or patch model [96-100]. Prior works were often based on an appropriate order parameter field (spin-density wave or nematic, for example), coupled with gapless fermions, residing close to the hot spots, through Yukawa coupling. Our approach can provide an alternative route to investigate the effects of strong electronic interactions on the Fermi surface, the role of competition among various incipient orderings, emergent symmetry near quantum critical points, etc. Very recently, it has also been argued that a topologically protected, multiflavored 2D anisotropic semimetal can also be realized in $\mathrm{Sr}_{2} \mathrm{IrO}_{4}$ [101]. When electron doped, $\mathrm{Sr}_{2} \mathrm{IrO}_{4}$ is believed to support high- $T_{c} d$-wave superconductivity [102,103]. Therefore, one can (at least in principle) generalize the formalism outlined here to address the intriguing confluence of electronic correlations, exotic broken-symmetry phases, competing orders, quantum critical phenomena, and the role of topology (such as in Weyl materials) in a wide variety of systems within a unified perturbatively controlled RG scheme.

\section{ACKNOWLEDGMENTS}

This work was supported by the Welch Foundation Grant No. C-1809 and by NSF CAREER Grant No. DMR1552327. Useful correspondence with Leon Balents and Fakher F. Assaad is thankfully acknowledged.

\section{APPENDIX A: OPTICAL CONDUCTIVITY IN THE ANISOTROPIC SEMIMETAL}

Due to the distinct power-law dependence of the quasiparticle dispersion along the $x$ and $y$ directions, the critical excitations show distinct scaling of the dynamic (frequency-dependent ac) conductivity along these two directions (denoted by $\sigma_{x x}$ and $\sigma_{y y}$, respectively). We use the Kubo formula to compute the dynamic conductivity. The expression for the polarization bubble is

$$
\begin{aligned}
\Pi_{j j}\left(i \omega_{n}\right)= & N \frac{e^{2}}{\beta} \sum_{m} \int_{\mathbf{k}} \operatorname{Tr}\left[\hat{J}_{j} G_{0}\left(\mathbf{k}, i p_{m}\right) \hat{J}_{j}\right. \\
& \left.\times G_{0}\left(\mathbf{k}, i p_{m}+i \omega_{n}\right)\right],
\end{aligned}
$$

where $e$ is the electronic charge; $\beta$ is the inverse temperature; $\omega_{n}, p_{m}$ are fermionic Matsubara frequencies; $\hat{J}_{x}=v \tau_{1} ; \hat{J}_{y}=2 b k_{y} \tau_{2}$; and $N$ counts the flavor degeneracy. Thus, for spinful fermions, $N=2$. The noninteracting Green's function reads as

$G_{0}\left(\mathbf{k}, i \omega_{n}\right)=\frac{1}{i \omega_{n}+\mu-H(\mathbf{k}, 0)}=\int_{-\infty}^{\infty} \frac{d \epsilon}{2 \pi} \frac{A(\mathbf{k}, \epsilon)}{i \omega_{n}-\epsilon}$,

where $\mu$ is the chemical potential and we have used the Lehmann representation in the final expression. The spectral function $A(\mathbf{k}, \omega)$ is given by

$$
\begin{aligned}
A(\mathbf{k}, \omega)= & \pi\left(1+\sum_{a=1}^{2} \tau_{a} \hat{d}_{a}\right) \delta\left(\omega+\mu-E_{\mathbf{k}}\right) \\
& +\pi\left(1-\sum_{a=1}^{2} \tau_{a} \hat{d}_{a}\right) \delta\left(\omega+\mu+E_{\mathbf{k}}\right),
\end{aligned}
$$

where $E_{\mathbf{k}}=\sqrt{v^{2} k_{x}^{2}+b^{2} k_{y}^{4}} ; \hat{d}_{a}=d_{a} / \sqrt{d_{1}^{2}+d_{2}^{2}}$ for $a=1$, 2; and $d_{1}=v k_{x}, d_{2}=b k_{y}^{2}$. The conductivity is defined as $\sigma_{j j}=-\operatorname{Im}\left[\Pi_{j j}(\omega)\right] / \omega$. The Drude conductivity (the contribution that comes only from the intraband piece) evaluates to Eq. (2), where $T_{0}=v^{2} /\left(k_{B} b\right)$ and

$$
\begin{aligned}
a_{x} & =\frac{K\left(\frac{1}{2}\right)}{3 \pi^{2}} \approx 0.06262, \quad a_{y}=\frac{3 \Gamma\left(-\frac{1}{4}\right)^{2}}{20 \pi^{5 / 2}} \approx 0.20602, \\
F_{n}(x) & =-\sum_{\sigma= \pm} \int_{0}^{\infty} d y y^{n-\frac{1}{2}} \operatorname{sech}^{2}(y+\sigma x) .
\end{aligned}
$$

The scaling of $F_{1}(x)$ and $F_{2}(x)$ is shown in Fig. 3(b). The result for the interband component of the optical conductivity is given by Eq. (3), where $\omega_{0}=v^{2} / b$. Thus, as the frequency $\omega \rightarrow 0$, the dynamic conductivity along the $y(x)$ direction vanishes (diverges) as $\sqrt{\omega}(1 / \sqrt{\omega})$. Such distinct power-law dependence of the conductivity along different directions can be directly measured in experiment to pin the quasiparticle dispersion. 


\section{APPENDIX B: DIAMAGNETIC SUSCEPTIBILITY}

Next we will discuss the effects of external magnetic fields on critical excitations and, in particular, we focus on the diamagnetic susceptibility. The Landau level (LL) spectrum of the ASM, described by the Hamiltonian $H(\mathbf{k}, 0)$, is $\pm E_{n}(B)$, where $n$ is the LL index [104] and, for $n=0,1,2, \cdots$,

$$
E_{n}(B)=A\left(\frac{2 v^{2}}{b}\right)^{1 / 3}\left[\omega_{c}\left(n+\frac{1}{2}\right)\right]^{2 / 3},
$$

with $A \approx 1.173, \omega_{c}=2 e B b$ is the cyclotron frequency, and $B$ is the strength of the external magnetic field [12]. The free energy at $T=0$ is defined as

$$
\begin{aligned}
\Omega_{0}(B) & =-C B \sum_{n=0}^{\infty} E_{n}(B)=-C \alpha B^{5 / 3} \sum_{n=0}^{\infty}\left(n+\frac{1}{2}\right)^{2 / 3} \\
& =-C \alpha B^{5 / 3} \zeta\left(-\frac{2}{3}, \frac{1}{2}\right) \\
& =-C \alpha B^{5 / 3}\left(2^{-2 / 3}-1\right) \zeta\left(-\frac{2}{3}\right) .
\end{aligned}
$$

Here $C=e / h$ and $C B$ counts the LL degeneracy. Note that $\Omega_{0}$ is a divergent quantity, which, however, can be regularized by using the zeta regulator as follows $[28,105,106]$ :

$$
\pi^{s} \zeta(1-s)=2^{1-s} \Gamma(s) \zeta(s) \cos \left(\frac{s \pi}{2}\right) .
$$

Upon using the above regularization scheme and the definition of the diamagnetic susceptibility $\chi=\partial^{2} \Omega_{0} / \partial B^{2}$, we arrive at the expression for the two-dimensional ASM at $T=0$,

$$
\chi=-\frac{\mathcal{A}}{B^{1 / 3}} \frac{e}{h}\left(e v \sqrt{\frac{b}{2}}\right)^{2 / 3} \equiv \chi_{0},
$$

where

$$
\mathcal{A}=\frac{10}{9} \zeta\left(-\frac{2}{3}\right)\left(2^{-2 / 3}-1\right) A \approx 0.075
$$

Notice that the diamagnetic susceptibility diverges as $B^{-1 / 3}$ as $B \rightarrow 0$.

The free energy at finite temperature reads as $\Omega(T)=$ $\Omega_{0}+\Omega_{T}$, where

$$
\Omega_{T}=-\frac{k_{B} T}{\pi \ell_{B}^{2}} \sum_{n=0}^{\infty} \log \left(1+e^{-\frac{E_{n}}{k_{B} T}}\right),
$$

and $\ell_{B}=\sqrt{\hbar /(e B)}$ is the magnetic length. After following the standard steps, highlighted above, we arrive at the following expression for the diamagnetic susceptibility at finite temperature $\chi(T)=\chi_{0}+\chi_{T}$, where $\chi_{T}=$ $\chi_{0} f\left(\lambda_{T h} / l_{B}\right)$ and

$$
\begin{aligned}
f(x) & =-\left(\frac{20}{9 \pi} \frac{A}{\mathcal{A}}\right) \times\left\{g(x)+\frac{3}{10} \frac{\partial}{\partial x} g(x)\right\} \\
& \approx-(11.063)\left\{g(x)+\frac{3}{10} \frac{\partial}{\partial x} g(x)\right\},
\end{aligned}
$$

with

$$
\begin{aligned}
\lambda_{T h} & =\frac{\hbar \sqrt{v}(b / 2)^{1 / 4}}{\left(k_{B} T\right)^{3 / 4}}, \quad x=\frac{\lambda_{T h}}{l_{B}} \sim \frac{\sqrt{B}}{T^{3 / 4}}, \\
g(x) & =\sum_{n=0}^{\infty} \frac{\left(n+\frac{1}{2}\right)^{2 / 3}}{e^{x^{4 / 3}\left(n+\frac{1}{2}\right)^{2 / 3}}+1} .
\end{aligned}
$$

Here, $\lambda_{T h}$ is the thermal de Broglie wavelength for the critical excitations. Our proposed scaling of the diamagnetic susceptibility at finite temperature is valid as long as $l_{B}<\lambda_{T h}<\xi_{\Delta}$, with $\xi_{\Delta} \sim 1 / \Delta$. Note that $f(x)$ is a universal function of dimensionless argument and, in Fig. 3(b), we have shown the scaling of $\log [-f(x)]$ vs. $\log [x]$.

\section{APPENDIX C: SYMMETRIES}

We summarize all symmetries of the noninteracting ASM defined via Eqs. (1) and (6). We use these to provide a more complete classification of the fermion bilinears in Table II and to connect to the Nambu notation in the scaling dimension Table V.

In terms of the four-component field $\Psi$ [Eq. (10)], sublattice symmetry (SLS) [Eq. (12)] and time-reversal invariance $(\mathcal{T})$ are both encoded as antiunitary transformations,

$$
\begin{aligned}
& \Psi(\boldsymbol{r}) \rightarrow \sigma_{0} \tau_{3}\left[\Psi^{\dagger}(\boldsymbol{r})\right]^{\top}, \\
& \Psi(\boldsymbol{r}) \rightarrow i \sigma_{2} \tau_{3} \Psi(\boldsymbol{r}), \quad i \rightarrow-i, \quad \text { SLS },
\end{aligned}
$$

where $\mathrm{T}$ denotes the transpose. We can also define the product of SLS and $\mathcal{T}$ as an effective particle-hole (PH) symmetry. This is implemented by the unitary transformation

$$
\Psi(\boldsymbol{r}) \rightarrow \sigma_{2} \tau_{0}\left[\Psi^{\dagger}(\boldsymbol{r})\right]^{\top}, \quad \mathrm{PH} .
$$

For the noninteracting problem, these imply the following conditions on the single-particle Hamiltonian in Eq. (6):

$$
\begin{aligned}
-\sigma_{0} \tau_{3} H_{n}(\mathbf{k}, \Delta) \sigma_{0} \tau_{3} & =H_{n}(\mathbf{k}, \Delta), & & \text { SLS }, \\
\sigma_{2} \tau_{3} H_{n}^{*}(-\mathbf{k}, \Delta) \sigma_{2} \tau_{3} & =H_{n}(\mathbf{k}, \Delta), & & \mathcal{T}, \\
-\sigma_{2} \tau_{0} H_{n}^{\top}(-\mathbf{k}, \Delta) \sigma_{2} \tau_{0} & =H_{n}(\mathbf{k}, \Delta), & & \text { PH. }
\end{aligned}
$$

We switch to the Nambu basis introduced in Eq. (37). This equation can also be written as 


$$
\Psi_{N}(\boldsymbol{r})=\left[\begin{array}{c}
\Psi(\boldsymbol{r}) \\
i \sigma_{2} \tau_{3}\left[\Psi^{\dagger}(\boldsymbol{r})\right]^{\top}
\end{array}\right]
$$

where the explicit decomposition is in the Nambu space, acted on by the Pauli matrices $\eta_{\mu}=\left\{\eta_{0}, \eta_{1}, \eta_{2}, \eta_{3}\right\}$. The Nambu spinor satisfies the reality condition

$$
\Psi_{N}^{\dagger}=\Psi_{N}^{\top} \eta_{2} \sigma_{2} \tau_{3}
$$

At the level of Bogoliubov-de Gennes (BdG) mean field theory, the Hamiltonian can be written as

$$
H_{\mathrm{BdG}}=\frac{1}{2} \Psi_{N}^{\dagger} h \Psi_{N}
$$

Here, $h$ is the single-particle $\mathrm{BdG}$ Hamiltonian. Equation (C4) implies that $h$ always satisfies the "automatic" particle-hole condition

$-M_{\mathrm{P}} h^{T} M_{\mathrm{P}}=h, \quad M_{\mathrm{P}}=\eta_{2} \sigma_{2} \tau_{3}, \quad$ Nambu-PH.

The symmetries in Eq. (C3) become, in the Nambu basis,

$$
\begin{array}{rrr}
-M_{\mathrm{S}} h M_{\mathrm{S}}=h, & M_{\mathrm{S}}=\eta_{3} \sigma_{0} \tau_{3}, & \text { SLS, } \\
M_{\mathrm{T}} h^{*} M_{\mathrm{\top}}=h, & M_{\mathrm{T}}=\eta_{0} \sigma_{2} \tau_{3}, & \mathcal{T}, \\
-M_{\mathrm{LP}} h^{T} M_{\mathrm{LP}}=h, & M_{\mathrm{LP}}=\eta_{1} \sigma_{0} \tau_{3}, & \text { PH. }
\end{array}
$$

We emphasize that there are two independent particlehole symmetries in this language. The Nambu condition in Eq. (C6) is not really a symmetry; it is merely a consequence of Pauli exclusion. Nevertheless, this condition implies that there are only 28 allowed independent fermion bilinears (without derivatives) in the Nambu basis, instead of 64. By contrast, the particle-hole condition in Eq. (C7c) is instead derived from the microscopic sublattice symmetry; it is not automatic. Finally, it is useful to synthesize an alternative version of time-reversal invariance, by combining the Nambu-PH condition [Eq. (C6)] with Eq. (C7b). This yields the chiral condition

$$
-\eta_{2} h \eta_{2}=h, \quad(\text { Nambu-PH }) \otimes \mathcal{T}
$$

Again, since the Nambu $\mathrm{PH}$ is automatic, Eq. (C8) is an equivalent definition of time-reversal symmetry.

The full inventory of 28 Nambu-PH Hermitian fermion bilinears appears in Table V. The properties of these bilinears under SLS, $\mathcal{T}, x$-reflection $\mathcal{R}_{\pi}$, and spin $\mathrm{SU}(2)$ symmetry transformations are summarized in Table II. To this, we add the properties under microscopic PH symmetry [Eqs. (C2) and (C7c)], shown in Table VI. We list only particle-hole channel bilinears.
TABLE VI. Symmetry of the particle-hole channel fermion bilinears listed in Table II under particle-hole (PH) transformation [Eqs. (C2) and (C7c)].

\begin{tabular}{lcc}
\hline \hline Bilinear & Description & PH \\
\hline$\Psi^{\dagger} \sigma_{0} \tau_{0} \Psi$ & Density & $\times$ \\
$\Psi^{\dagger} \sigma_{0} \tau_{1} \Psi$ & $x$ current & $\times$ \\
$\Psi^{\dagger} \sigma_{0} \tau_{2} \Psi$ & AP & $\checkmark$ \\
$\Psi^{\dagger} \sigma_{0} \tau_{3} \Psi$ & CDW & $\times$ \\
$\Psi^{\dagger} \vec{\sigma} \tau_{0} \Psi$ & Ferromagnet & $\checkmark$ \\
$\Psi^{\dagger} \vec{\sigma} \tau_{1} \Psi$ & spin current & $\checkmark$ \\
$\Psi^{\dagger} \vec{\sigma} \tau_{2} \Psi$ & Spin BDW & $\times$ \\
$\Psi^{\dagger} \vec{\sigma} \tau_{3} \Psi$ & AFM & $\checkmark$ \\
\hline \hline
\end{tabular}

\section{APPENDIX D: 1D LIMIT AND REVIEW OF SPIN-CHARGE SEPARATION}

We translate the anisotropic semimetal model into the standard notations for $1 \mathrm{D}$ physics. After a $\tau_{2}$-rotation that sends $\tau_{1} \rightarrow \tau_{3}$, the field in Eq. (10) is decomposed into right- $R$ and left- $L$ mover components via $\Psi^{\top}=[R, L]$, leading to

$\mathcal{S}_{0}=\int d^{2} \mathbf{r} d t\left[\begin{array}{c}\bar{R}\left(\partial_{t}-i v \partial_{x}\right) R+\bar{L}\left(\partial_{t}+i v \partial_{x}\right) L \\ -i b\left(\bar{R} \partial_{y}^{n} L-\bar{L} \partial_{y}^{n} R\right)\end{array}\right]$.

For the case of spin-1/2 electrons, the chiral components $R$ and $L$ are each two-component spinors.

The four four-fermion interaction operators appearing in Eq. (17) are chosen to exploit spin-charge separation $[42,43]$ in the $1 \mathrm{D}(n \rightarrow \infty)$ limit. These are defined as

$$
\begin{aligned}
\mathcal{O}_{N} & \equiv(\bar{R} \vec{\sigma} R) \cdot(\bar{L} \vec{\sigma} L), \\
\mathcal{O}_{A} & \equiv(\bar{R} R)(\bar{L} L), \\
\mathcal{O}_{W} & \equiv\left(\bar{R} \sigma_{2} \bar{R}^{\top}\right)\left(L^{\top} \sigma_{2} L\right), \\
\overline{\mathcal{O}}_{W} & \equiv\left(\bar{L} \sigma_{2} \bar{L}^{\top}\right)\left(R^{\top} \sigma_{2} R\right), \\
\mathcal{O}_{X} & \equiv(\bar{R} R)^{2}+(\bar{L} L)^{2} .
\end{aligned}
$$

The operator $\mathcal{O}_{N}$ is an $\mathrm{SU}(2)_{1}$ current-current perturbation; the remaining operators couple only to the $U(1)$ electric charge sector [43].

In the $1 \mathrm{D}$ limit, we can bosonize the U(1) charge and spin $\mathrm{SU}(2)_{1}$ sectors; up to irrelevant operators, these completely decouple [43]. The imaginary time bosonic action for the charge sector can be written as

$S_{c}=\int d^{2} \boldsymbol{r} d t\left[\begin{array}{c}i \partial_{x} \theta \partial_{t} \phi+\frac{v_{c}}{2 K_{c}}\left(\partial_{x} \theta\right)^{2}+\frac{v_{c} K_{c}}{2}\left(\partial_{x} \phi\right)^{2} \\ -\frac{2 W}{(2 \pi)^{2}} \cos (\sqrt{8 \pi} \theta)\end{array}\right]$

where $\phi(\theta)$ denotes the polar (axial) field, and the charge velocity $v_{c}$ and Luttinger parameter $K_{c}$ are given by 
TABLE VII. Scaling dimensions of all fermion bilinears in the 1D limit $(\epsilon=0, n \rightarrow \infty)$, in the stable gapless phase consisting of independent spin and charge Luttinger liquids; cf. Fig. 4(a). The common dimensions for the AP, CDW, spin-BDW, and AFM bilinears are the sum of the spin $(1 / 2)$ and charge $\left(K_{c} / 2\right)$ sector contributions; the same applies to the $s$-wave SC and triplet SC. The chiral $\mathrm{SC}_{1,2}$ bilinears reside entirely in the charge sector. We ignore logarithmic corrections to the spin sector attributable to nonzero, but marginally irrelevant $U_{N}<0$ [42].

\begin{tabular}{lcc}
\hline \hline Bilinear & Description & Operator scaling dimension \\
\hline$\Psi^{\dagger} \sigma_{0} \tau_{0} \Psi$ & Density & 1 \\
$\Psi^{\dagger} \sigma_{0} \tau_{1} \Psi$ & $x$ current & 1 \\
$\Psi^{\dagger} \sigma_{0} \tau_{2} \Psi$ & AP & $(1 / 2)+\left(K_{c} / 2\right)$ \\
$\Psi^{\dagger} \sigma_{0} \tau_{3} \Psi$ & CDW & $(1 / 2)+\left(K_{c} / 2\right)$ \\
$\Psi^{\dagger} \vec{\sigma} \tau_{0} \Psi$ & Ferromagnet & 1 \\
$\Psi^{\dagger} \vec{\sigma} \tau_{1} \Psi$ & $x$ spin current & $(1 / 2)+\left(K_{c} / 2\right)$ \\
$\Psi^{\dagger} \vec{\sigma} \tau_{2} \Psi$ & Spin BDW & $(1 / 2)+\left(K_{c} / 2\right)$ \\
$\Psi^{\dagger} \vec{\sigma} \tau_{3} \Psi$ & AFM & $(1 / 2)+\left(1 / 2 K_{c}\right)$ \\
$\Psi \sigma_{2} \tau_{3} \Psi$ & s-wave SC & $\left(K_{c}+1 / K_{c}\right) / 2$ \\
$\Psi \sigma_{2} \tau_{1} \Psi$ & Chiral SC & $\left(K_{c}+1 / K_{c}\right) / 2$ \\
$\Psi \sigma_{2} \tau_{0} \Psi$ & Chiral SC & $(1 / 2)+\left(1 / 2 K_{c}\right)$ \\
$\Psi \sigma_{(0,1,3)} \tau_{2} \Psi$ & Triplet SC & \\
\hline \hline
\end{tabular}

$$
v_{c}=v(1+X), \quad K_{c}=1-U_{A} / 2 .
$$

The $\mathrm{U}(1)$ current-current operator $\mathcal{O}_{A}$ and $\mathrm{U}(1)$ stress tensor operator $\mathcal{O}_{X}$ are completely absorbed into these parameters of the free boson, while the umklapp operator $\frac{1}{2}\left(\mathcal{O}_{W}+\overline{\mathcal{O}}_{W}\right)$ becomes a sine-Gordon perturbation. As usual $[42,43]$, the expressions for $v_{c}$ and $K_{c}$ in terms of the microscopic coupling strengths $U_{A}$ and $X$ obtained via perturbative bosonization are valid only to first order.

When both charge and spin sectors are gapless, the theory asymptotes to a conformal fixed point in the infrared. This is a product of spin and charge Luttinger liquids [cf. Fig. 4(a)]. The SU(2) invariant fixed point has $U_{N}=W=0$, with $U_{A}<0\left(K_{c}>1\right)$. The exact scaling dimensions of all fermion bilinears in Tables II and V at this fixed point are summarized in Table VII.

\section{APPENDIX E: TABLE OF INTEGRALS}

To proceed with the perturbative $\mathrm{RG}$ analysis to the leading order in $\epsilon$ and $1 / n$, we need to evaluate the following integrals:

$$
\begin{aligned}
& I_{1}=\int \frac{\omega^{2}}{\left(\omega^{2}+v^{2} k_{x}^{2}+b^{2} k_{y}^{2 n}\right)^{2}}, \\
& I_{2}=\int \frac{v^{2} k_{x}^{2}}{\left(\omega^{2}+v^{2} k_{x}^{2}+b^{2} k_{y}^{2 n}\right)^{2}}, \\
& I_{3}=\int \frac{b^{2} k_{y}^{2 n}}{\left(\omega^{2}+v^{2} k_{x}^{2}+b^{2} k_{y}^{2 n}\right)^{2}}, \\
& I_{4}=\int \frac{b k_{y}^{n}}{\left(\omega^{2}+v^{2} k_{x}^{2}+b^{2} k_{y}^{2 n}\right)} .
\end{aligned}
$$

The relevant Feynman diagrams are shown in Figs. 6 and 10 . We introduce a new set of variables, defined as

$$
\omega=\rho \cos \theta, \quad v k_{x}=\rho \sin \theta, \quad x=\frac{b^{1 / n} k_{y}}{E_{\Lambda}^{1 / n}},
$$

where $\rho=\sqrt{\omega^{2}+v^{2} k_{x}^{2}}$, and $E_{\Lambda}$ is the ultraviolet energy cutoff. Within the framework of the Wilsonian RG, we integrate out the fast Fourier modes within the shell $E_{\Lambda} e^{-l}<$ $\rho<E_{\Lambda}$ and subsequently integrate over $0 \leq \theta \leq 2 \pi$ and $0 \leq x \leq \infty$. The above integrals are then given by

$$
\begin{aligned}
I_{1} & =I_{2}=a_{\epsilon} l \int_{0}^{\infty} d x \frac{1}{\left(1+x^{2 n}\right)^{2}} \\
& =a_{\epsilon} l\left[\frac{\pi(2 n-1) \csc \left(\frac{\pi}{2 n}\right)}{4 n^{2}}\right] \\
& =a_{\epsilon}\left(1-\frac{1}{2 n}\right) l+\mathcal{O}\left(\frac{1}{n^{2}}\right) \\
I_{3} & =a_{\epsilon} l \int_{0}^{\infty} d x \frac{2 x^{2 n}}{\left(1+x^{2 n}\right)^{2}} \\
& =a_{\epsilon} l\left[\frac{\pi \csc \left(\frac{\pi}{2 n}\right)}{2 n^{2}}\right]=a_{\epsilon}\left(\frac{1}{n}\right) l+\mathcal{O}\left(\frac{1}{n^{2}}\right) \\
I_{4} & =E_{\Lambda} a_{\epsilon} l \int_{0}^{\infty} d x \frac{2 x^{n}}{1+x^{2 n}} \\
& =E_{\Lambda} a_{\epsilon} l\left[\frac{\pi \sec \left(\frac{\pi}{2 n}\right)}{n}\right] \\
& =E_{\Lambda} a_{\epsilon}\left(\frac{\pi}{n}\right) l+\mathcal{O}\left(\frac{1}{n^{2}}\right)
\end{aligned}
$$

where $a_{\epsilon} \equiv E_{\Lambda}^{\epsilon} /\left(8 \pi^{2} v b^{\epsilon}\right)$. Thus, as $n \rightarrow \infty$, only the contributions from $I_{1}$ and $I_{2}$ survive. We note that all integrals over $x$ are convergent for any $n \geq 2$.

\section{APPENDIX F: FIERZ IDENTITY}

In this appendix, we present the Fierz constraints among local four-fermion terms that allow us to extract the set of linearly independent quartic terms in an interacting model. We discuss this subject for spinless fermions and spin-1/2 electrons separately.

\section{Spinless fermions}

To find the number of independent coupling constants for spinless fermions, we define a four-dimensional vector as

$X_{4}^{\top}=\left[\left(\psi^{\dagger} \tau_{0} \psi\right)^{2},\left(\psi^{\dagger} \tau_{1} \psi\right)^{2},\left(\psi^{\dagger} \tau_{2} \psi\right)^{2},\left(\psi^{\dagger} \tau_{3} \psi\right)^{2}\right]$ 
The Pauli matrices $\tau_{\mu}=\left\{\tau_{0}, \tau_{1}, \tau_{2}, \tau_{3}\right\}$ constitute a complete basis for $2 \times 2$ matrices. The Fierz constraint for two component spinor $(\psi)$ reads as

$$
\begin{aligned}
& {\left[\psi^{\dagger}(x) \tau_{a} \psi(x)\right]\left[\psi^{\dagger}(y) \tau_{b} \psi(y)\right]} \\
& \quad=-\frac{1}{4} \operatorname{Tr}\left[\tau_{a} \tau_{c} \tau_{b} \tau_{d}\right]\left[\psi^{\dagger}(x) \tau_{c} \psi(y)\right]\left[\psi^{\dagger}(y) \tau_{d} \psi(x)\right],
\end{aligned}
$$

where $a, b, c, d=0,1,2,3$ and, for local interaction, $x=y$. The above Fierz constraint can then be compactly written as $F_{4 \times 4} X_{4}=0$, where

$$
F_{4 \times 4}=\left[\begin{array}{cccc}
3 & 1 & 1 & 1 \\
1 & 3 & -1 & -1 \\
1 & -1 & 3 & -1 \\
1 & -1 & -1 & 3
\end{array}\right] .
$$

The rank of $F_{4 \times 4}$ is 3 and, therefore, the number of linearly independent coupling constants is $4-3=1$. Thus, we can choose $\left(\psi^{\dagger} \tau_{3} \psi\right)^{2}$ as the independent interaction operator and the remaining quartic terms are related according to

$$
-\left(\psi^{\dagger} \tau_{0} \psi\right)^{2}=\left(\psi^{\dagger} \tau_{1} \psi\right)^{2}=\left(\psi^{\dagger} \tau_{2} \psi\right)^{2}=\left(\psi^{\dagger} \tau_{3} \psi\right)^{2} .
$$

\section{Spin-1/2 electrons}

To find the number of linearly independent coupling constants in the interacting Hamiltonian for spin-1/2 electrons, we first define an eight-component vector as

$$
\begin{aligned}
X_{8}^{\top}= & {\left[\left(\Psi^{\dagger} \sigma_{0} \tau_{0} \Psi\right)^{2},\left(\Psi^{\dagger} \sigma_{0} \tau_{1} \Psi\right)^{2},\left(\Psi^{\dagger} \sigma_{0} \tau_{2} \Psi\right)^{2},\right.} \\
& \left(\Psi^{\dagger} \sigma_{0} \tau_{3} \Psi\right)^{2},\left(\Psi^{\dagger} \vec{\sigma} \tau_{0} \Psi\right)^{2},\left(\Psi^{\dagger} \vec{\sigma} \tau_{1} \Psi\right)^{2}, \\
& \left.\left(\Psi^{\dagger} \vec{\sigma} \tau_{2} \Psi\right)^{2},\left(\Psi^{\dagger} \vec{\sigma} \tau_{3} \Psi\right)^{2}\right] .
\end{aligned}
$$

The Fierz constraint reads as

$$
\begin{aligned}
& {\left[\Psi^{\dagger}(x) M \Psi(x)\right]\left[\Psi^{\dagger}(y) N \Psi(y)\right]} \\
& \quad=-\frac{1}{16} \operatorname{Tr}\left[M \Gamma_{a} N \Gamma_{b}\right]\left[\Psi^{\dagger}(x) \Gamma_{a} \Psi(y)\right]\left[\Psi^{\dagger}(y) \Gamma_{b} \Psi(x)\right],
\end{aligned}
$$

which we apply here for contact interaction $x=y$. Here, $M$ and $N$ are $4 \times 4$ Hermitian matrices, and $\Gamma_{a}$ closes the basis for all $4 \times 4$ Hermitian matrices, $a, b=1, \ldots, 16$. Thus, the Fierz identity allows us to write each quartic term as a linear combination of rest. The above constraint can then be compactly written as $F_{8 \times 8} X_{8}=0$, where

$$
F_{8 \times 8}=\left[\begin{array}{cccccccc}
5 & 1 & 1 & 1 & 1 & 1 & 1 & 1 \\
1 & 5 & -1 & -1 & 1 & 1 & -1 & -1 \\
1 & -1 & 5 & -1 & 1 & -1 & 1 & -1 \\
1 & -1 & -1 & 5 & 1 & -1 & -1 & 1 \\
3 & 3 & 3 & 3 & 3 & -1 & -1 & -1 \\
3 & 3 & -3 & -3 & -1 & 3 & 1 & 1 \\
3 & -3 & 3 & -3 & -1 & 1 & 3 & 1 \\
3 & -3 & -3 & 3 & -1 & 1 & 1 & 3
\end{array}\right] .
$$

The rank of the Fierz matrix $F_{8 \times 8}$ is four and, therefore, the number of linearly independent four-fermion local interactions is $8-4=4$. We choose $\left(\Psi^{\dagger} \sigma_{0} \tau_{1} \Psi\right)^{2},\left(\Psi^{\dagger} \sigma_{0} \tau_{3} \Psi\right)^{2}$, $\left(\Psi^{\dagger} \vec{\sigma} \tau_{2} \Psi\right)^{2}$, and $\left(\Psi^{\dagger} \vec{\sigma} \tau_{3} \Psi\right)^{2}$ as linearly independent quartic terms in $H_{\text {int }}$, as shown in Eq. (16). The rest of the quartic terms can then be expressed as a linear combination of the above four according to

$$
\begin{aligned}
\left(\Psi^{\dagger} \sigma_{0} \tau_{0} \Psi\right)^{2}= & \left(\Psi^{\dagger} \sigma_{0} \tau_{1} \Psi\right)^{2}-\left(\Psi^{\dagger} \sigma_{0} \tau_{3} \Psi\right)^{2} \\
& -\frac{1}{3}\left(\Psi^{\dagger} \vec{\sigma} \tau_{2} \Psi\right)^{2}-\frac{2}{3}\left(\Psi^{\dagger} \vec{\sigma} \tau_{3} \Psi\right)^{2}, \\
\left(\Psi^{\dagger} \sigma_{0} \tau_{2} \Psi\right)^{2}= & \left(\Psi^{\dagger} \sigma_{0} \tau_{3} \Psi\right)^{2}-\frac{1}{3}\left(\Psi^{\dagger} \vec{\sigma} \tau_{2} \Psi\right)^{2}+\frac{1}{3}\left(\Psi^{\dagger} \vec{\sigma} \tau_{3} \Psi\right)^{2}, \\
\left(\Psi^{\dagger} \vec{\sigma} \tau_{0} \Psi\right)^{2}= & -3\left(\Psi^{\dagger} \sigma_{0} \tau_{1} \Psi\right)^{2}+\left(\Psi^{\dagger} \vec{\sigma} \tau_{2} \Psi\right)^{2}+\left(\Psi^{\dagger} \vec{\sigma} \tau_{3} \Psi\right)^{2}, \\
\left(\Psi^{\dagger} \vec{\sigma} \tau_{1} \Psi\right)^{2}= & -3\left(\Psi^{\dagger} \sigma_{0} \tau_{1} \Psi\right)^{2}+3\left(\Psi^{\dagger} \sigma_{0} \tau_{3} \Psi\right)^{2}+\left(\Psi^{\dagger} \vec{\sigma} \tau_{3} \Psi\right)^{2} .
\end{aligned}
$$

In the RG calculation, whenever we generate any one of these terms, it is rewritten back in terms of the four quartic terms appearing in $H_{\text {int }}$ [Eq. (16)].

\section{APPENDIX G: CLIFFORD ALGEBRA OF MASS MATRICES IN THE 2D ASM}

We here provide an algebraic derivation of the internal algebra among various mass orders in the twodimensional ASM (such as the CDW, Néel AFM, and $s$-wave pairing). This analysis is purely based on the Clifford algebra of real matrices and does not rely on specific microscopic details of the model. Let us define a Nambu-doubled spinor as $\Psi=\left(\Psi_{p}, \Psi_{h}\right)^{\top}$, so that the effective Hamiltonian describing low energy excitations in an ASM assumes the form

$$
H_{\mathrm{ASM}}(\mathbf{k})=H_{0}(\mathbf{k}) \oplus\left[-H_{0}^{\top}(-k)\right],
$$

where $H_{0}(\mathbf{k})=\sum_{j=1,2} \alpha_{j} d_{j}(\mathbf{k})$, with

$$
d_{1}(\mathbf{k})=v k_{x}, \quad d_{2}(\mathbf{k})=b k_{y}^{2} .
$$

Here, $\alpha_{1}$ and $\alpha_{2}$ are two mutually anticommuting fourcomponent Hermitian matrices. Next, we wish to find all 
the Hermitian eight-dimensional matrices $(M$ 's) that anticommute with $H_{\mathrm{ASM}}(\mathbf{k})$, such that $m=\left\langle\Psi^{\dagger} M \Psi\right\rangle \neq 0$. Therefore, $M$ 's represent mass matrices. Such a condition is satisfied only when

$$
M=-\left(\sigma_{1} \otimes \mathbb{I}_{4}\right) M^{\top}\left(\sigma_{1} \otimes \mathbb{I}_{4}\right),
$$

where $\mathbb{}_{4}$ is a four-dimensional identity matrix. There

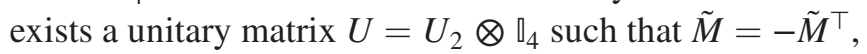
where $\tilde{M}=U M U^{\dagger}$ and

$$
U_{2}=\sqrt{ \pm i} e^{i \frac{\pi}{4} \sigma_{3}} e^{i \frac{\pi}{4} \sigma_{2}} e^{i\left(\frac{\pi}{4}-\phi\right) \sigma_{3}}
$$

Therefore, after the unitary transformation, all mass matrices are purely imaginary $[107,108]$.

Let us now write two mutually anticommuting fourdimensional matrices, appearing in $H_{0}(\mathbf{k})$ as

$$
\alpha_{j}=\Re\left(\alpha_{j}\right)+i \Im\left(\alpha_{j}\right),
$$

for $j=1,2$. After such decomposition, the kinetic energy of the ASM assumes the form

$$
\begin{aligned}
H_{\mathrm{ASM}}(\mathbf{k})= & {\left[\sigma_{0} \otimes \Re\left(\alpha_{1}\right)+i \sigma_{3} \otimes \Im\left(\alpha_{1}\right)\right] d_{1}(\mathbf{k}) } \\
& +\left[\sigma_{3} \otimes \Re\left(\alpha_{2}\right)+i \sigma_{0} \otimes \Im\left(\alpha_{2}\right)\right] d_{2}(\mathbf{k}) .
\end{aligned}
$$

Since $U_{2} \sigma_{3} U_{2}^{\dagger}=\sigma_{2}$, after the unitary transformation with $U$, the kinetic energy becomes

$$
\begin{aligned}
\tilde{H}_{\mathrm{ASM}}(\mathbf{k})= & {\left[\sigma_{0} \otimes \Re\left(\alpha_{1}\right)+i \sigma_{2} \otimes \Im\left(\alpha_{1}\right)\right] d_{1}(\mathbf{k}) } \\
& +\left[\sigma_{2} \otimes \Re\left(\alpha_{2}\right)+i \sigma_{0} \otimes \Im\left(\alpha_{2}\right)\right] d_{2}(\mathbf{k}) \\
= & \tilde{\Gamma}_{1} d_{1}(\mathbf{k})+\tilde{\Gamma}_{2} d_{2}(\mathbf{k}) .
\end{aligned}
$$

Thus, $\tilde{\Gamma}_{1}$ and $\tilde{\Gamma}_{2}$ are, respectively, mutually anticommuting, but purely real and imaginary eight-dimensional Hermitian matrices. We seek all purely imaginary matrices $(\tilde{M})$ that anticommute with $\tilde{\Gamma}_{1}$ and $\tilde{\Gamma}_{2}$. Since $i \tilde{\Gamma}_{2}$ and $i \tilde{M}$ are purely real and square to -1 , while $\tilde{\Gamma}_{1}$ is purely real and squares to +1 , we seek to know the maximal number of $q$ so that for $p \geq 1$, the dimensionality of real matrices is eight and, together, they close the $C(p, q)$ algebra. Notice that the $C(p, q)$ Clifford algebra is defined by a set of $p+q$ mutually anticommuting matrices, among which $p$ of them square to +1 , while $q$ of them square to -1 . The answer is $q=4$ and $p=1[107,109]$. Thus, the maximal number of mutually anticommuting matrices is five, and we define a set of such five matrices as $\left\{R, I_{1}, I_{2}, I_{3}, I_{4}\right\}$, where $R$ is real and the $I_{j}$ 's are imaginary for $j=1, \ldots, 4$. Two of those five matrices, say, $R$ and $I_{1}$, can be used to define the kinetic energy. Therefore, $I_{2}, I_{3}$, and $I_{4}$ represent three mass matrices that, together, close a $\mathrm{Cl}(3)$ algebra, defining a set of three mutually anticommuting matrices. However, we have not exhausted all possible mass matrices for a 2D ASM yet.

Note that the Clifford algebra $C(1,4)$ follows the quaternionic representation that supports three real Casimir operators, $K_{j}$ for $j=1,2,3$, besides the standard identity matrix. The Casimir operators satisfy the quaternionic algebra

$$
K_{i} K_{j}=-\delta_{i j}+\epsilon_{i j k} K_{k},
$$

where $\delta_{i j}$ is the Kronecker delta function. From the Casimir operators, we can define imaginary matrices $E_{j}=i K_{j}$ for $j=1,2,3$, and $E_{j}$ 's satisfy the $\mathrm{SU}(2)$ algebra. Notice that $E_{j}$ 's are purely imaginary Hermitian matrices that commute with $H_{\mathrm{ASM}}(\mathbf{k})$. From these three imaginary matrices, we can define yet another set of three mutually anticommuting matrices $M_{j}^{\prime}=i E_{j} R I_{1}$ for $j=1,2,3$ that, together, close a $\mathrm{Cl}(3)$ algebra and also anticommute with $H_{\mathrm{ASM}}(\mathbf{k})$. Hence, there are all together six purely imaginary matrices (thus, mass matrices) that anticommute with $H_{\mathrm{ASM}}(\mathbf{k})$, and they can be arranged into two sets according to

$$
\left\{I_{2}, I_{3}, I_{4}\right\} \quad \text { and } \quad\left\{M_{1}^{\prime}, M_{2}^{\prime}, M_{3}^{\prime}\right\}
$$

Together, these two sets close a $C l(3) \times C l(3)$ algebra of mass matrices, as we announced in the main part of the paper. One set we can identify with the three components of Néel AFM order, while the other set is constituted by the CDW and two components (real and imaginary) of an $s$-wave pairing. We note that the Clifford algebra of mass matrices in a 2D ASM is identical to that for a threedimensional line-node semimetal [108].

[1] K. S. Novoselov, A. K. Geim, S. V. Morozov, D. Jiang, M. I. Katsnelson, I. V. Grigorieva, S. V. Dubonos, and A. A. Firsov, Two-Dimensional Gas of Massless Dirac Fermions in Graphene, Nature (London) 438, 197 (2005).

[2] Y. Zhang, Y.-W. Tan, H. L. Störmer, and P. Kim, Experimental Observation of the Quantum Hall Effect and Berry's Phase in Graphene, Nature (London) 438, 201 (2005).

[3] For a review, see A. H. Castro Neto, F. Guinea, N. M. R. Peres, K. S. Novoselov, and A. K. Geim, The Electronic Properties of Graphene, Rev. Mod. Phys. 81, 109 (2009).

[4] For $\Delta>0$, there is no real solution of $k_{y}$, implying that the phase is an insulator.

[5] There is no symmetry distinction between a Dirac semimetal and band insulator.

[6] H. Isobe, B.-J. Yang, A. Chubukov, J. Schmalian, and N. Nagaosa, Emergent Non-Fermi-Liquid at the Quantum Critical Point of a Topological Phase Transition in Two Dimensions, Phys. Rev. Lett. 116, 076803 (2016).

[7] G. Y. Cho and E.-G. Moon, Novel Quantum Criticality in Two Dimensional Topological Phase Transitions, Sci. Rep. 6, 19198 (2016). 
[8] For all possible mass orders and their symmetry properties in the graphene-based Dirac semimetal, see S. Ryu, C. Mudry, C.-Y. Hou, and C. Chamon, Masses in Graphenelike Two-Dimensional Electronic Systems: Topological Defects in Order Parameters and Their Fractional Exchange Statistics, Phys. Rev. B 80, 205319 (2009).

[9] Y. Hasegawa, R. Konno, H. Nakano, and M. Kohmoto, Zero Modes of Tight-Binding Electrons on the Honeycomb Lattice, Phys. Rev. B 74, 033413 (2006).

[10] P. Dietl, F. Piéchon, and G. Montambaux, New Magnetic Field Dependence of Landau Levels in a Graphenelike Structure, Phys. Rev. Lett. 100, 236405 (2008); G. Montambaux, F. Piéchon, J.-N. Fuchs, and M. O. Goerbig, Merging of Dirac Points in a Two-Dimensional Crystal, Phys. Rev. B 80, 153412 (2009).

[11] V. M. Pereira, A. H. Castro Neto, and N. M. R. Peres, Tight-Binding Approach to Uniaxial Strain in Graphene, Phys. Rev. B 80, 045401 (2009).

[12] G. Montambaux, F. Piéchon, J. Fuchs, and M. O. Goerbig, A Universal Hamiltonian for Motion and Merging of Dirac Points in a Two-Dimensional Crystal, Eur. Phys. J. B 72, 509 (2009).

[13] L. Tarruell, D. Greif, T. Uehlinger, G. Jotzu, and T. Esslinger, Creating, Moving and Merging Dirac Points with a Fermi Gas in a Tunable Honeycomb Lattice, Nature (London) 483, 302 (2012).

[14] T. Uehlinger, G. Jotzu, M. Messer, D. Greif, W. Hofstetter, U. Bissbort, and T. Esslinger, Artificial Graphene with Tunable Interactions, Phys. Rev. Lett. 111, 185307 (2013).

[15] M. Tarnowski, M. Nuske, N. Fläschner, B. Rem, D. Vogel, L. Freystatzky, K. Sengstock, L. Mathey, and C. Weitenberg, Observation of Topological Bloch-State Defects and Their Merging Transition, Phys. Rev. Lett. 118, 240403 (2017).

[16] K. Bender, K. Dietz, H. Endres, H. W. Helberg, I. Hennig, H. J. Keller, H. W. Schäfer, and D. Schweitzer, (BEDT-TTF) ${ }_{2} \mathrm{~J}_{3}^{-}$: A Two-Dimensional Organic Metal, Mol. Cryst. Liq. Cryst. 107, 45 (1984).

[17] K. Bender, I. Hennig, D. Schweitzer, K. Dietz, H. Endres, and H.J. Keller, Synthesis, Structure and Physical Properties of a Two-Dimensional Organic Metal, Di[bis(ethylenedithiolo)tetrathiofulvalene] Triiodide, $(\text { BEDT-TTF })_{2}^{+} \mathbf{J}_{3}^{-}$, Mol. Cryst. Liq. Cryst. 108, 359 (1984).

[18] S. Katayama, A. Kobayashi, and Y. Suzumura, PressureInduced Zero-Gap Semiconducting State in Organic Conductor $\alpha$-(BEDT-TTF $)_{2} \mathrm{I}_{3}$ Salt, J. Phys. Soc. Jpn. 75, 054705 (2006).

[19] A. Kobayashi, S. Katayama, Y. Suzumura, and H. Fukuyama, Massless Fermions in Organic Conductor, J. Phys. Soc. Jpn. 76, 034711 (2007).

[20] M. Ezawa, Topological Origin of Quasi-Flat Edge Band in Phosphorene, New J. Phys. 16, 115004 (2014).

[21] J. Kim, S. S. Baik, S. H. Ryu, Y. Sohn, S. Park, B.-G. Park, J. Denlinger, Y. Yi, H. J. Choi, and K. S. Kim, Observation of Tunable Band Gap and Anisotropic Dirac Semimetal State in Black Phosphorus, Science 349, 723 (2015).

[22] H. Doh and H. J. Choi, Dirac-Semimetal Phase Diagram of Two-Dimensional Black Phosphorus, 2D Mater. 4, 025071 (2017).
[23] V. Pardo and W. E. Pickett, Half-Metallic Semi-DiracPoint Generated by Quantum Confinement in $\mathrm{TiO}_{2} / \mathrm{VO}_{2}$, Phys. Rev. Lett. 102, 166803 (2009); Metal-Insulator Transition through a Semi-Dirac Point in Oxide Nanostructures: $\mathrm{VO}_{2}(001)$ Layers Confined within $\mathrm{TiO}_{2}$, Phys. Rev. B 81, 035111 (2010).

[24] S. Banerjee, R. R. P. Singh, V. Pardo, and W. E. Pickett, Tight-Binding Modeling and Low-Energy Behavior of the Semi-Dirac Point, Phys. Rev. Lett. 103, 016402 (2009).

[25] M. Polini, F. Guinea, M. Lewenstein, H. C. Manoharan, and V. Pellegrini, Artificial Honeycomb Lattices for Electrons, Atoms and Photons, Nat. Nanotechnol. 8, 625 (2013).

[26] D. Liu, K. Ishikawa, R. Takehara, K. Miyagawa, M. Tamura, and K. Kanoda, Insulating Nature of Strongly Correlated Massless Dirac Fermions in an Organic Crystal, Phys. Rev. Lett. 116, 226401 (2016).

[27] For discussion on scaling properties in a Dirac semimetal, see D. E. Sheehy and J. Schmalian, Quantum Critical Scaling in Graphene, Phys. Rev. Lett. 99, 226803 (2007).

[28] A. Ghosal, P. Goswami, and S. Chakravarty, Diamagnetism of Nodal Fermions, Phys. Rev. B 75, 115123 (2007).

[29] P. Goswami and S. Chakravarty, Quantum Criticality between Topological and Band Insulators in $3+1$ Dimensions, Phys. Rev. Lett. 107, 196803 (2011).

[30] B. Roy and J. D. Sau, Magnetic Catalysis and Axionic Charge Density Wave in Weyl Semimetals, Phys. Rev. B 92, 125141 (2015).

[31] In other words, we require $\ell_{B}<\xi_{\Delta}$, where $\xi_{\Delta} \sim 1 / \Delta$ is the correlation length associated with the Dirac-semimetal to band-insulator QCP and $\ell_{B} \sim B^{-1 / 2}$ is the magnetic length, so that the magnetic length provides the infrared cutoff. The above condition is required to observe the scaling of the diamagnetic susceptibility $\chi_{0}$, reported in Eq. (4).

[32] By contrast, the thermal de Broglie wavelength for relativistic and nonrelativistic fermions scales as $\lambda_{T h} \sim$ $T^{-1}$ and $T^{-1 / 2}$, respectively.

[33] The scaling of the diamagnetic susceptibility for the anisotropic semimetal at finite temperature and weak magnetic field (no Landau quantization) can be estimated as follows: $\chi(T) \sim B^{-1 / 3} \sim \ell_{B}^{2 / 3} \sim \lambda_{T h}^{2 / 3}$, yielding $\chi(T) \sim T^{-1 / 2}$. On the other hand, for a two-dimensional Dirac semimetal, the diamagnetic susceptibility at finite $T$ scales as $\chi(T) \sim B^{-1 / 2} \sim \ell_{B} \sim \lambda_{t h}$, yielding $\chi(T) \sim T^{-1}$.

[34] J. Zinn-Justin, Quantum Field Theory and Critical Phenomena (Oxford University Press, Oxford, U.K., 2002).

[35] An additional advantage of our $\epsilon$ scheme is that it is performed in fixed $d=2$ spatial dimensions; no analytic continuation of the Clifford algebra is required.

[36] A comparison can be drawn between our combined $\epsilon$ and $1 / n$ expansion with a conventional double expansion in $\epsilon$ and $1 / N$, where $N$ is the flavor number of the original problem [37]. Typically, $\epsilon$ captures the deviation from the marginal dimension. However, the parameter $1 / N$ only controls the quantum loop corrections and does not enter in the engineering dimension of the coupling $[g]=-\epsilon$. By contrast, we here gain control over loop corrections by tuning the band curvature in the $k_{y}$ direction (through the parameter $1 / n$, which does not enter the scaling dimension 
of $g$, namely, $[g]=-\epsilon$, in $\left.d_{*}=1+\epsilon\right)$ instead of artificially increasing the flavor number.

[37] M. Moshe and J. Zinn-Justin, Quantum Field Theory in the Large N Limit: A Review, Phys. Rep. 385, 69 (2003).

[38] For a review, see, e.g., H. Q. Lin, D. K. Campbell, and R. T. Clay, Broken Symmetries in the One-Dimensional Extended Hubbard Model, Chin. J. Phys. (Taipei) 38, 1 (2000).

[39] M. Tsuchiizu and A. Furusaki, Phase Diagram of the OneDimensional Extended Hubbard Model at Half Filling, Phys. Rev. Lett. 88, 056402 (2002).

[40] A. W. Sandvik, L. Balents, and D. K. Campbell, Ground State Phases of the Half-Filled One-Dimensional Extended Hubbard Model, Phys. Rev. Lett. 92, 236401 (2004).

[41] F. Iemini, T. O. Maciel, and R. O. Vianna, Entanglement of Indistinguishable Particles as a Probe for Quantum Phase Transitions in the Extended Hubbard Model, Phys. Rev. B 92, 075423 (2015).

[42] T. Giamarchi, Quantum Physics in One Dimension (Clarendon Press, Oxford, England, 2003).

[43] A. M. Tsvelik, Quantum Field Theory in Condensed Matter Physics 2nd ed. (Cambridge University Press, Cambridge, England, 2007).

[44] C. N. Yang and S. C. Zhang, $\mathrm{SO}_{4}$ Symmetry in a Hubbard Model, Mod. Phys. Lett. B 04, 759 (1990).

[45] A. Auerbach, Interacting Electrons and Quantum Magnetism (Springer-Verlag, New York, NY, 1994).

[46] For a review of pseudospin $S U(2)$ symmetry in the honeycomb lattice Hubbard model, see, e.g., M. Hermele, SU(2) Gauge Theory of the Hubbard Model and Application to the Honeycomb Lattice, Phys. Rev. B 76, 035125 (2007).

[47] The Dirac-semimetal to band-insulator QCP in the uniaxially strained honeycomb lattice is achieved at the cost of $C_{3 v}$ symmetry (present in the absence of strain); see Fig. 1(b). Thus, the coupling $V$ is expected to be different for the three NN sites. Such modification can only lead to a nonuniversal shift of the phase boundaries in Fig. 4(b), without altering its qualitative and universal features.

[48] T. Esslinger, Fermi-Hubbard Physics with Atoms in an Optical Lattice, Annu. Rev. Condens. Matter Phys. 1, 129 (2010).

[49] T. O. Wehling, E. Şaş̧oğlu, C. Friedrich, A. I. Lichtenstein, M. I. Katsnelson, and S. Blügel, Strength of Effective Coulomb Interactions in Graphene and Graphite, Phys. Rev. Lett. 106, 236805 (2011).

[50] H. H. P. Garza, E. W. Kievit, G. F. Schneider, and U. Staufer, Controlled, Reversible, and Nondestructive Generation of Uniaxial Extreme Strains (> 10\%) in Graphene, Nano Lett. 14, 4107 (2014).

[51] R. Bistritzer and A.H. MacDonald, Moiré Bands in Twisted Double-Layer Graphene, Proc. Natl. Acad. Sci. U.S.A. 108, 12233 (2011); K. Kim, A. DaSilva, S. Huang, B. Fallahazad, S. Larentisa, T. Taniguchi, K. Watanabe, B. J. LeRoy, A. H. MacDonald, and E. Tutuc, Tunable Moiré Bands and Strong Correlations in Small-TwistAngle Bilayer Graphene, Proc. Natl. Acad. Sci. U.S.A. 114, 3364 (2017).
[52] R. E. Throckmorton and O. Vafek, Fermions on Bilayer Graphene: Symmetry Breaking for $B=0$ and $\nu=0$, Phys. Rev. B 86, 115447 (2012).

[53] C. Tonnoir, A. Kimouche, J. Coraux, L. Magaud, B. Delsol, B. Gilles, and C. Chapelier, Induced Superconductivity in Graphene Grown on Rhenium, Phys. Rev. Lett. 111, 246805 (2013).

[54] A. Di Bernardo, O. Millo, M. Barbone, H. Alpern, Y. Kalcheim, U. Sassi, A. K. Ott, D. De Fazio, D. Yoon, M. Amado, A. C. Ferrari, J. Linder, and J. W. A. Robinson, p-Wave Triggered Superconductivity in Single-Layer Graphene on an Electron-Doped Oxide Superconductor, Nat. Commun. 8, 14024 (2017).

[55] The half-filled Hubbard model on a bipartite lattice (with $V=0$ ) exhibits exact pseudospin SU(2) symmetry [44-46].

[56] Thus, for the physically relevant system (strained honeycomb lattice), $\nu=2$ to the leading order, distinct from the result for the Dirac-semimetal to broken-symmetry-phase transition characterized by $\nu=1$ (to the leading order).

[57] S. Sorella, Y. Otsuka, and S. Yunoki, Absence of a Spin Liquid Phase in the Hubbard Model on the Honeycomb Lattice, Sci. Rep. 2, 992 (2012).

[58] F. F. Assaad and I. F. Herbut, Pinning the Order: The Nature of Quantum Criticality in the Hubbard Model on Honeycomb Lattice, Phys. Rev. X 3, 031010 (2013).

[59] F. P. Toldin, M. Hohenadler, F. F. Assaad, and I. F. Herbut, Fermionic Quantum Criticality in Honeycomb and $\pi$-flux Hubbard Models: Finite-Size Scaling of RenormalizationGroup-Invariant Observables from Quantum Monte Carlo, Phys. Rev. B 91, 165108 (2015).

[60] Y. Otsuka, S. Yunoki, and S. Sorella, Universal Quantum Criticality in the Metal-Insulator Transition of TwoDimensional Interacting Dirac Electrons, Phys. Rev. X 6, 011029 (2016).

[61] S. Pujari, T. C. Lang, G. Murthy, and R. K. Kaul, Interaction-Induced Dirac Fermions from Quadratic Band Touching in Bilayer Graphene, Phys. Rev. Lett. 117, 086404 (2016).

[62] K. L. Lee, K. Bouadim, G. G. Batrouni, F. Hébert, T. R. Scalettar, C. Miniatura, and B. Grémaud, Attractive Hubbard Model on a Honeycomb Lattice: Quantum Monte Carlo Study, Phys. Rev. B 80, 245118 (2009).

[63] L. Wang, P. Corboz, and M. Troyer, Fermionic Quantum Critical Point of Spinless Fermions on a Honeycomb Lattice, New J. Phys. 16, 103008 (2014).

[64] Z.-X. Li, Y.-F. Jiang, and H. Yao, Fermion-Sign-Free Majorana-Quantum-Monte-Carlo Studies of Quantum Critical Phenomena of Dirac Fermions in Two Dimensions, New J. Phys. 17, 085003 (2015).

[65] B. Roy, P. Goswami, and V. Juričić, Interacting Weyl Fermions: Phases, Phase Transitions, and Global Phase Diagram, Phys. Rev. B 95, 201102(R) (2017).

[66] I. F. Herbut, V. Juričić, and B. Roy, Theory of Interacting Electrons on the Honeycomb Lattice, Phys. Rev. B 79, 085116 (2009).

[67] M. S. Foster and I. L. Aleiner, Graphene via Large N: A Renormalization Group Study, Phys. Rev. B 77, 195413 (2008). 
[68] L. V. Keldysh and Y. V. Kopaev, Possible Instability of Semimetallic State Toward Coulomb Interaction, Sov. Phys. Solid State 6, 2219 (1965).

[69] T. M. Rice, Band-Structure Effects in Itinerant Antiferromagnetism, Phys. Rev. B 2, 3619 (1970).

[70] I. L. Aleiner, D. E. Kharzeev, and A. M. Tsvelik, Spontaneous Symmetry Breaking in Graphene Subjected to an In-Plane Magnetic Field, Phys. Rev. B 76, 195415 (2007).

[71] B. Roy, J. Hofmann, V. Stanev, J. D. Sau, and V. Galitski, Excitonic and Nematic Instabilities on the Surface of Topological Kondo Insulators, Phys. Rev. B 92, 245431 (2015).

[72] For the clarity of presentation, we here denote the spinor for spinless (two-component) and spinful (four-component) fermions as $\psi$ and $\Psi$, respectively.

[73] Even though here we do not account for any local or spatial fluctuation of the order parameter $\Sigma$ or $\sigma$ field, the terminology "fluctuation-driven first-order transition" is justified in this case, since it can only be captured upon incorporating quantum corrections to the free-energy density $(f)$ due to electronic interaction $g_{2}$ or $\lambda_{2}$. In particular, in the large- $N$ (flavor number) limit, such corrections arise from the Feynman diagrams (b) and (d), shown in Fig. 6. See also Ref. [74] for discussion of a similar issue in one spatial dimension.

[74] D. J. Gross and A. Neveu, Dynamical Symmetry Breaking in Asymptotically Free Field Theories, Phys. Rev. D 10, 3235 (1974); G. S. Grest and M. Widom, N-Color Ashkin-Teller Model, Phys. Rev. B 24, 6508 (1981); E. Fradkin, $N$-Color Ashkin-Teller Model in Two Dimensions: Solution in the Large-N Limit, Phys. Rev. Lett. 53, 1967 (1984); R. Shankar, Ashkin-Teller and Gross-Neveu Models: New Relations and Results, Phys. Rev. Lett. 55, 453 (1985).

[75] B. Roy, P. Goswami, and J. D. Sau, Continuous and Discontinuous Topological Quantum Phase Transitions, Phys. Rev. B 94, 041101(R) (2016).

[76] A. Amaricci, J. C. Budich, M. Capone, B. Trauzettel, and G. Sangiovanni, First-Order Character and Observable Signatures of Topological Quantum Phase Transitions, Phys. Rev. Lett. 114, 185701 (2015).

[77] A. Amaricci, J. C. Budich, M. Capone, B. Trauzettel, and G. Sangiovanni, Strong Correlation Effects on Topological Quantum Phase Transitions in Three Dimensions, Phys. Rev. B 93, 235112 (2016).

[78] See, also, V. Juričić, D. S. L. Abergel, and A. V. Balatsky, First-Order Quantum Phase Transition in ThreeDimensional Topological Band Insulators, Phys. Rev. B 95, 161403 (2017).

[79] For a similar discussion related to the determination of the location of fixed points in a $\Phi^{4}$ theory within the framework of an $\epsilon(=4-d)$ expansion, see P. M. Chaikin and T.C. Lubensky, Principles of Condensed Matter Physics (Cambridge University Press, Cambridge, England, 2000), Chap. 5.

[80] J. A. Gracey, Computation of Critical Exponent $\eta$ at $\mathcal{O}\left(1 / N^{3}\right)$ in the Four-Fermi Model in Arbitrary Dimensions, Int. J. Mod. Phys. A 09, 727 (1994).

[81] L. Rosa, P. Vitale, and C. Wetterich, Critical Exponents of the Gross-Neveu Model from the Effective Average Action, Phys. Rev. Lett. 86, 958 (2001).
[82] F. Höfling, C. Nowak, and C. Wetterich, Phase Transition and Critical Behavior of the $d=3$ Gross-Neveu Model, Phys. Rev. B 66, 205111 (2002).

[83] J.-R. Wang, G.-Z. Liu, and C.-J. Zhang, Excitonic Pairing and Insulating Transition in Two-Dimensional Semi-Dirac Semimetals, Phys. Rev. B 95, 075129 (2017).

[84] J. Cardy, Scaling and Renormalization in Statistical Physics (Cambridge University Press, Cambridge, England, 1996).

[85] B. Roy and I. F. Herbut, Unconventional Superconductivity on Honeycomb Lattice: The Theory of Kekule Order Parameter, Phys. Rev. B 82, 035429 (2010); B. Roy, Classification of Massive and Gapless Phases in Bilayer Graphene, Phys. Rev. B 88, 075415 (2013).

[86] For the discussion on the restoration of symmetry among various orders at interacting quantum critical points for Dirac fermions, see, e.g., B. Roy, Multicritical Behavior of $Z_{2} \otimes O(2)$ Gross-Neveu-Yukawa Theory in Graphene, Phys. Rev. B 84, 113404 (2011); B. Roy and V. Juričić, Strain-Induced Time-Reversal Odd Superconductivity in Graphene, Phys. Rev. B 90, 041413(R) (2014).

[87] A. Mazurenko, C. S. Chiu, G. Ji, M. F. Parsons, M KanászNagy, R. Schmidt, F. Grusdt, E. Demler, D. Greif, and M. Greiner, A Cold-Atom Fermi-Hubbard Antiferromagnet, Nature (London) 545, 462 (2017).

[88] B. Rosenstein, H.-L. Yu, and A. Kovner, Critical Exponents of New Universality Classes, Phys. Lett. B 314, 381 (1993).

[89] I. F. Herbut, V. Juricic, and O. Vafek, Relativistic Mott Criticality in Graphene, Phys. Rev. B 80, 075432 (2009).

[90] G. Xu, H. Weng, Z. Wang, X. Dai, and Z. Fang, Chern Semimetal and the Quantized Anomalous Hall Effect in $\mathrm{HgCr}_{2} \mathrm{Se}_{4}$, Phys. Rev. Lett. 107, 186806 (2011).

[91] C. Fang, M.J. Gilbert, X. Dai, and B. A. Bernevig, Multi-Weyl Topological Semimetals Stabilized by Point Group Symmetry, Phys. Rev. Lett. 108, 266802 (2012).

[92] B.-J. Yang and N. Nagaosa, Classification of Stable ThreeDimensional Dirac Semimetals with Nontrivial Topology, Nat. Commun. 5, 4898 (2014).

[93] B. Roy, R.-J. Slager, and V. Juričić, Global Phase Diagram of a Dirty Weyl Semimetal, arXiv:1610.08973.

[94] The effect of Dirac cone anisotropy on electronic interactions has been addressed via mean-field analysis in A. Sharma, V. N. Kotov, and A. H. Castro Neto, Excitonic Mass Gap in Uniaxially Strained Graphene, Phys. Rev. B 95, 235124 (2017).

[95] S. Sachdev, Quantum Phase Transitions (Cambridge University Press, Cambridge, England, 2007), 2nd ed.

[96] J. Polchinski, Effective Field Theory and the Fermi Surface, arXiv:hep-th/9210046.

[97] Typically, a Fermi surface is unstable against sufficiently weak backscattering toward the formation of a superconductor. See, for example, R. Shankar, RenormalizationGroup Approach to Interacting Fermions, Rev. Mod. Phys. 66, 129 (1994).

[98] M. A. Metlitski and S. Sachdev, Quantum Phase Transitions of Metals in Two Spatial Dimensions. I. Ising-Nematic Order, Phys. Rev. B 82, 075127 (2010). 
[99] M. A. Metlitski and S. Sachdev, Quantum Phase Transitions of Metals in Two Spatial Dimensions. II. Spin Density Wave Order, Phys. Rev. B 82, 075128 (2010).

[100] S. Sur and S.-S. Lee, Chiral Non-Fermi Liquids, Phys. Rev. B 90, 045121 (2014).

[101] M. Horsdal and T. Hyart, Robust Semi-Dirac Points and Unconventional Topological Phase Transitions in Doped SuperConducting $\mathrm{Sr}_{2} \mathrm{IrO}_{4}$ Tunnel Coupled to $2 \mathrm{~g}$ Electron Systems, SciPost Phys. 3, 041 (2017).

[102] Y. J. Yan, M. Q. Ren, H. C. Xu, B. P. Xie, R. Tao, H. Y. Choi, N. Lee, Y. J. Choi, T. Zhang, and D. L. Feng, Electron-Doped $\mathrm{Sr}_{2} \mathrm{IrO}_{4}$ : An Analogue of Hole-Doped Cuprate Superconductors Demonstrated by Scanning Tunneling Microscopy, Phys. Rev. X 5, 041018 (2015).

[103] Y. K. Kim, N. H. Sung, J. D. Denlinger, and B. J. Kim, Observation of a d-Wave Gap in Electron-Doped $\mathrm{Sr}_{2} \mathrm{IrO}_{4}$, Nat. Phys. 12, 37 (2016).

[104] The LL index $n$ in this appendix should not be confused with the anisotropy parameter introduced in Eq. (6).
[105] E. Elizalde, S. D. Odintsov, A. Romeo, A. A. Bytsenko, and S. Zerbini, Zeta Regularization Techniques with Applications (World Scientific, Singapore, 1994).

[106] B. Roy, M. P. Kennett, and S. Das Sarma, Chiral Symmetry Breaking and the Quantum Hall Effect in Monolayer Graphene, Phys. Rev. B 90, 201409(R) (2014); B. Roy and J.D. Sau, Magnetic Catalysis and Axionic Charge Density Wave in Weyl Semimetals, Phys. Rev. B 92, 125141 (2015); B. Roy, Theory of Integer Quantum Hall Effect in Insulating Bilayer Graphene, Phys. Rev. B 89, 201401(R) (2014); B. Roy, Z.-X. Hu, and K. Yang, Theory of Unconventional Quantum Hall Effect in Strained Graphene, Phys. Rev. B 87, 121408(R) (2013).

[107] I. F. Herbut, Isospin of Topological Defects in Dirac Systems, Phys. Rev. B 85, 085304 (2012).

[108] B. Roy, Interacting Nodal-Line Semimetal: Proximity Effect and Spontaneous Symmetry Breaking, Phys. Rev. B 96, 041113(R) (2017).

[109] S. Okubo, Real Representations of Finite Clifford Algebras. I. Classification, J. Math. Phys. (N.Y.) 32, 1657 (1991). 